\title{
Objective Video Quality Assessment using the 3D Dual-Tree Complex Wavelet Transform
}

\author{
by \\ Khalil S. Yonis, B. Eng.

\begin{abstract}
A Thesis submitted to
the Faculty of Graduate Studies and Research

in partial fulfilment of

the requirements for the degree of

Master of Applied Science in Electrical Engineering

Ottawa-Carleton Institute for

Electrical and Computer Engineering
\end{abstract}

Department of Systems and Computer Engineering

Carleton University

Ottawa, Ontario, Canada

April 2009

Copyright (C)

2009 - Khalil S. Yonis 
Library and

Archives Canada

Published Heritage

Branch

395 Wellington Street

Ottawa ON K1A 0N4

Canada
Bibliothèque et

Archives Canada

Direction du

Patrimoine de l'édition

395 , rue Wellington

Ottawa ON K1A 0N4

Canada
Your file Votre référence

ISBN: 978-0-494-52043-7

Ourfile Notre référence

ISBN: 978-0-494-52043-7
NOTICE:

The author has granted a nonexclusive license allowing Library and Archives Canada to reproduce, publish, archive, preserve, conserve, communicate to the public by telecommunication or on the Internet, loan, distribute and sell theses worldwide, for commercial or noncommercial purposes, in microform, paper, electronic and/or any other formats.

The author retains copyright ownership and moral rights in this thesis. Neither the thesis nor substantial extracts from it may be printed or otherwise reproduced without the author's permission.
AVIS:

L'auteur a accordé une licence non exclusive permettant à la Bibliothèque et Archives Canada de reproduire, publier, archiver, sauvegarder, conserver, transmettre au public par télécommunication ou par l'Internet, prêter, distribuer et vendre des thèses partout dans le monde, à des fins commerciales ou autres, sur support microforme, papier, électronique et/ou autres formats.

L'auteur conserve la propriété du droit d'auteur et des droits moraux qui protège cette thèse. $\mathrm{Ni}$ la thèse ni des extraits substantiels de celle-ci ne doivent être imprimés ou autrement reproduits sans son autorisation.
In compliance with the Canadian

Privacy Act some supporting forms may have been removed from this thesis.

While these forms may be included in the document page count, their removal does not represent any loss of content from the thesis.
Conformément à la loi canadienne sur la protection de la vie privée, quelques formulaires secondaires ont été enlevés de cette thèse.

Bien que ces formulaires aient inclus dans la pagination, il n'y aura aucun contenu manquant.

\section{Canadä}




\section{Abstract}

In this thesis, we propose using the dual-tree complex wavelet transform (DT-CWT) with the complex wavelet structural similarity (CW-SSIM) index for image quality assessment. Also, we present a video quality measure that is formed as an extension of the $\mathbb{C W}$-SSIM measure by instead using a three-dimensional DT- $\mathbb{C W T}$. The near shift invariance of the $\mathbb{C W}$-SSIM has been used for image quality and pattern recognition. We take advantage of the near shift invariance and directional selectivity of the 3-D DT-CWT to represent not only frame structure, but also motion through the frames in a similar vein as recent 3-D DT-CWT scalable video coders.

As we did not find a full evaluation of the CW-SSIM algorithm, we used the LIVE image database to test and evaluate the $\mathbb{C W}$-SSIM against other algorithms. Also, we used video sequences from the video quality experts group (VQEG) multimedia project to evaluate the performance of the proposed 3D-DT-CW-SSIM extension.

Our test results indicated that the $\mathbb{C W}$-SSIM algorithm has a very good performance in image quality assessment, with the use of the DT-CWT elevating its quality prediction accuracy. The proposed 3-D extension to the $\mathbb{C W}$-SSIM algorithm showed good performance with low motion video sequences. 
I would like to dedicate this work to my mother, my father, and my brothers.

Thank you all, you made this possible. 


\section{Acknowledgments}

I would like to thank both my parents, Said Yonis and Fayzeh Zeidan, as well as my brothers, Ramzi, Omar, and Sami, for their love, endless support, and encouragement throughout my research period.

I would like to acknowledge and express my deepest gratitude to my supervisor, professor Richard M. Dansereau, who was abundantly helpful and offered invaluable assistance, support and guidance, without which this research project would not have been possible.

Special thanks goes to the graduate assistant, Ms. Anna Lee, who provided me with constant help and assistance since the first day. 


\section{Table of Contents}

Abstract $\quad$ iii

Acknowledgments $\quad$ v

Table of Contents vi vi

List of Tables $\quad$ x

List of Figures $\quad$ xi

1 Introduction $\quad 1$

1.1 Introduction . . . . . . . . . . . . . . . . 1

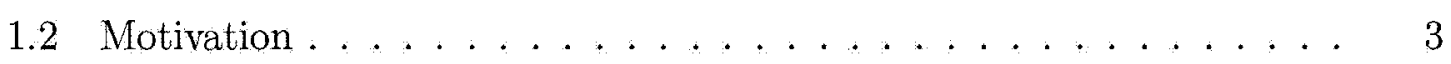

1.3 Statement of the Problem .................. 4

1.4 Contributions .......................... 5

1.5 Overview of Results . . . . . . . . . . . . . . 6

1.6 Organization of Thesis ................... 7

$\begin{array}{llr}2 & \text { Background } & 8\end{array}$

2.1 The Human Visual System (HVS) . . . . . . . . . . . . 8

2.1.1 The multi-channel structure of the HVS . . . . . . . . . 9

2.1.2 The contrast sensitivity of the HVS . . . . . . . . . 9

vi 
2.1.3 The masking effect in the HVS ............. 10

2.2 Distortions that can occur in Digital Images and Videos . . . . . . . . 11

2.3 Subjective Quality Assessment . . . . . . . . . . . . . . . . . . 13

2.4 Objective Quality Assessment ... . . . . . . . . . . 17

2.5 Perceptual Quality Assessment - General Framework . . . . . . . 18

2.6 Image Quality Assessment . . . . . . . . . . . . . . . . 20

2.6 .1 Element based algorithms . . . . . . . . . . . 21

2.6 .2 Fourier magnitude difference . . . . . . . . . . . . . 22

2.6.3 Magnitude and phase model based on Gervais et al. . . . . . 23

2.6 .4 Visual difference predictor ................ 23

2.6.5 Model after Daly ... . . . . . . . . . . 25

2.6 .6 Visual discrimination model . . . . . . . . . . . . . 26

2.6.7 Gabor pyramid visible difference predictor . . . . . . . . . 27

2.6.8 Wavelet image quality measure . . . . . . . . . . 27

2.6.9 Wavelet visible difference predictor . . . . . . . . . . 29

2.6.10 Multistage perceptual quality assessment . . . . . . . . 29

2.7 Video Quality Assessment . . . . . . . . . . . . . . 31

2.7.1 Emulating human perception with objective video quality mea-

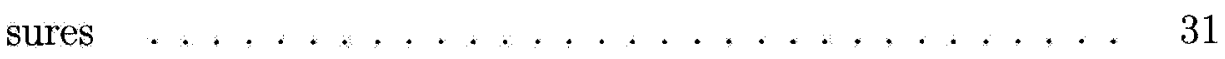

2.7.2 The moving pictures quality metric . . . . . . . . . 33

2.7.3 Sarnoff's just noticeable difference (JND) . . . . . . . . . . 34

2.7.4 Watson's digital video quality metric . . . . . . . . . 35

2.7.5 Winkler's perception difference metric . . . . . . . . . 36

2.7.6 NTIA general video quality assessment model . . . . . . . 38

2.7.7 Wavelet-based video visible distortion measure . . . . . . 42

2.7.8 Motion models based video structural similarity metric . . . . 44 
3 The Structural Similarity Index $\quad 47$

3.1 SSIM Index: An Image Quality Assessment Algorithm . . . . . . . 50

3.2 Moving SSIM to the Complex Wavelet Domain . . . . . . . . . . 56

3.3 SSIM in Video Quality Assessment . . . . . . . . . . . . . . 61

4 Dual-Tree Complex Wavelet Transform 65

4.1 Wavelet Transform . . . . . . . . . . . . . . . . 65

4.2 Dual-Tree Complex Wavelet Transform . . . . . . . . . . . . . . 69

5 Image Quality Assessment using the Dual-Tree Complex Wavelet $\begin{array}{ll}\text { SSIM } & \mathbf{7 6}\end{array}$

5.1 Image Quality Assessment in the Dual-Tree Domain . . . . . . . . . 77

5.2 LIVE Image Database . . . . . . . . . . . . . . . . . 78

5.3 Implementations and Results . . . . . . . . . . . . . . 80

5.4 Perceptually Weighted $\mathbb{C W}$-SSIM Index . . . . . . . . . . . 88

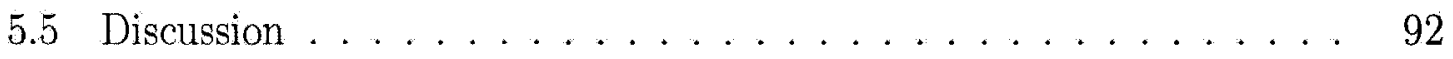

6 Video Quality Assessment using the Dual-Tree Complex Wavelet $\begin{array}{ll}\text { SSIM } & 96\end{array}$

6.1 Why use the $3-\mathrm{D}$ DT-CWT? . . . . . . . . . . 96

6.2 Proposed Approach . . . . . . . . . . . . . . . . . . . . . 98

6.3 Test Material . . . . . . . . . . . . . . . . 100

6.4 Implementation and Testing . . . . . . . . . . . . . . . 101

6.5 Discussion .......................... 104

7 Conclusions and Future Work 105

7.1 Conclusions . . . . . . . . . . . . . . . . 105

7.2 Future Work . . . . . . . . . . . . . . . 107

viii 
List of References 


\section{List of Tables}

5.1 Objective quality tests' performance for JPEG and JPEG2000 images 86

5.2 Objective quality tests' performance for all LIVE images . . . . . . . 86

5.3 Weighted CW-SSIM performance for JPEG and JPEG2000 images . 89

5.4 Weighted CW-SSIM performance for all LIVE images . . . . . . . . 89

6.1 3D-DT-CW-SSIM test results for VQEG's MM video set. . . . . . . . 103

6.2 3D-DT-CW-SSIM test results for low motion VQEG's MM video se-

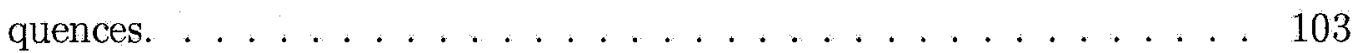




\section{List of Figures}

2.1 Typical HVS perceptual channel decomposition . . . . . . . . . 10

2.2 Examples of some image and video distortions . . . . . . . . . . . 14

2.3 Examples of generalized grading scale sheets used with subjective tests 15

2.4 DSCQS basic test cell . . . . . . . . . . . . . 16

2.5 Generalized perception-based objective assessment block diagram . . 18

2.6 Visual difference predictor ................... 24

2.7 Visual discrimination model . . . . . . . . . . . . . . . 26

2.8 Gabor pyramid visible difference predictor . . . . . . . . . . 28

2.9 Multistage perceptual quality assessment . . . . . . . . . . . 30

2.10 Voran's objective quality algorithm derivation process . . . . . . . . 31

2.11 Perception difference metric . . . . . . . . . . . . 37

3.1 SSIM quality index example results . . . . . . . . . . . . . . 51

3.2 Diagram of the structural similarity (SSIM) algorithm . . . . . . . 52

3.3 SSIM quality map example . . . . . . . . . . . . . . 56

3.4 Comparison of image similarity measures for images with different

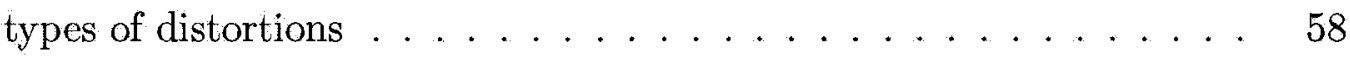

3.5 Video Structural Similarity (video-SSIM) . . . . . . . . . 61

4.1 Multi-scale discrete wavelet transform . . . . . . . . . . . . . 67

4.2 Image decomposition using Discrete wavelet transform . . . . . . 68 
4.3 1-D D'T-CWTanalysis filter bank ............... . 70

4.4 Typical 2-D DWT and DT-CWTwavelet bases . . . . . . . . 72

4.5 Typical 3-D DWT and DT-CWTwavelet bases . . . . . . . . . 75

5.1 LIVE database reference images' thumbnails . . . . . . . . . . . . . 81

$5.2 \quad \mathbb{C W}$-SSIM scatter plots for JPEG and JPEG2000 images . . . . . 85

5.3 CW-SSIM scatter plots for all LIVE images . . . . . . . . . . . 87

5.4 Weighted CW-SSIM scatter plots for JPEG and JPEG2000 images . 90

5.5 Weighted CW-SSIM scatter plots for all LIVE images . . . . . . . . . 91

5.6 Disk image discomposed using both the steerable pyramid transform and the D'-CWT . . . . . . . . . . . . . . . . 94

6.1 Low motion VQEG MM project videos . . . . . . . . . . . . 103 


\section{Chapter 1}

\section{Introduction}

\section{$1.1 \quad$ Introduction}

We humans are information driven beings. For that purpose, we are equipped with multiple sensory systems that allow us to collect information about our surroundings. The Human Visual System (HVS) is one of the most complicated and well developed information collection and processing systems in our possession. It allows us to collect information under a wide range of conditions and about different objects, small or large, close or far, still or in motion. Its information extraction capabilities allows for object recognition even if viewing conditions have changed or if the object it self changed its location and/or orientation.

Images can provide a great amount of information. Hence, humans documented their existence and life stories throughout history, even before any of the known civilizations came about, using drawing. This fascination in preserving information as images lead to early studies in capturing and preserving natural scenes and resulted in the first camera (camera obscura) by Ibn Al-Haytham (Alhazen) which was described is his Book Kitab al-Manazir (The Book of Optics). Although Ibn Al-Haytham provided a good description of a camera to capture images, his camera and ones 
latter developed were not capable of capturing permanent images. It was not until 1862 where the first permanent image is considered to be taken by Joseph Nicphore Nipce. Since then, photography has come a long way and along with the later, motion pictures (video), occupied a very important roll in our information driven world.

The development of commercial digital cameras in the early 1990 's, and the advances in computer technologies and networking opened many new research doors in digital image and video processing that includes but is not limited to: image and video acquisition, compression, media storage and digital image/video transmission.

Another important research field that is related to images and videos is quality assessment. Quality assessment is a very crucial element that must be considered in any image and/or video processing algorithm. The quality of the final product has a great effect on the amount of processing the image or video will undergo and can be sometimes the sole target to be met by the processing algorithm.

To assess the quality of an image or a video, two different approaches can be used. The first approach is the subjective quality assessment (SQA). Through SQA, human subjects judge and rate the product's final quality. The second method of quality testing is objective quality assessment (OQA). With the OQA approach, computer algorithms are used to estimate the quality of the product instead of human subjects. With human viewers as the final receiver for video technology, subjective video quality assessment can yield the perfect and desired quality measure. However, due to subjective test preparation requirements, cost, and time consumption, there is a demand for objective tests that can automatically evaluate the quality of the produced digital images and videos in a more feasible manner.

OQA is divided into three categories based on the reference ${ }^{1}$ availability. The three quality assessment algorithm categories are: full reference (FR), reduced reference

\footnotetext{
${ }^{1}$ The reference here is the original image or video sequence that is normally uncompressed and/or is considered to be of $100 \%$ quality
} 
(RR) and no-reference (NR) [1]. In this thesis, we will discuss FR objective quality assessment algorithms related to digital image and video signals. Also, we will present a novel approach for assessing digital videos in the complex wavelet domain. The rest of this chapter is organized in the following manner: the following section will discuss the motivation behind this research. After the motivation, the statement of the problem is presented followed by our contribution and an overview of the research results. The thesis break down is presented in the last section of the chapter.

\subsection{Motivation}

For a long time, video was in an analog format and its quality was measured by analyzing and comparing the frequency response of the video signal. The development of digital video did not only replace its analog predecessor, but with the developments in computers, networking and video equipped devices, became an increasingly popular and important part of many services such as video-on-demand, digital cinema, video conferencing, security, etc. This rabid increase in demand for video enabled technologies sprouted many research efforts to develop compression, processing, and communication techniques to meet user requirements. However, this increase in digital video technology was not matched with similar progress in quality assessment algorithms [2].

The new advances in the video compression and playback devices resulted in a new generation of consumers that have new and higher standards of what is acceptable in terms of video quality. Also, with many technologies highly affected by and affecting the quality of the final digital video, the need for a quantitative measure to rate the quality of videos can be very crucial to the development and use of many digital video algorithms. 
Being able to successfully estimate the quality of a digital video is very important to any research that involves video compression and/or transmission. For that reason, we felt that studying and understanding some of the available approaches in this field as well as contributing to the field by designing a new objective quality assessment approach will be very beneficial to any later research related to digital video processing. We have elected to focus our research work on the structural similarity (SSIM) quality index [3] (Chapter 3), as it is one of the latest and highly rated, yet simple FR objective quality assessment algorithms. Along side our study of the SSIM algorithm and its extensions, we have decided to implement a video SSIM quality algorithm operating in the complex wavelet domain instead of the pixel domain (as the initial implementation of the video SSIM was).

\subsection{Statement of the Problem}

The most commonly used Full Reference (FR) objective quality tests are the mean square error (MSE) and the peak signal-to-noise ratio (PSNR). This is mainly due to their simplicity of calculation and their clear physical meaning. However, both MSE and PSNR do not reflect a true quality metric and do not correlate well with subjective test scores [4-6]. This lack of high correlation resulted in a number of research efforts that tried to simulate the HVS in evaluating digital image and video quality [7-16]. However, more recent work in the area of FR objective image and video quality was focused on calculating the quality based on the visual characteristics of the media, without imitating or simulating the HVS directly $[2,3,17-21]$.

Although the quality of video can be evaluated in a similar fashion to digital images, when evaluated in a frame by frame fashion, a successful video quality algorithm 
must account for motion information and temporal artifacts in its assessment. However, motion evaluation adds a great deal of complexity to any algorithm, increases the algorithm's computational time as well as the algorithm processing and memory requirements.

Due to the lack of high correlation between subjective results and MSE/PSNR outputs, the complexity of simulating the HVS, and the computation cost of motion evaluation, there is a need for an objective video quality assessment algorithm that can have: (i) a clear physical meaning, (ii) can account for some of the HVS characteristics without implicitly simulating them, and (iii) can account for temporal changes in the video without having to estimate the motion directly.

In this thesis, we propose a new FR objective video quality metric for digital videos that does not require motion estimation. The algorithm, which is an extension to the complex wavelet structure similarity index (CW-SSIM) proposed in [20], utilizes the $3 \mathrm{D}$ dual-tree complex wavelet transform $(\mathrm{DT}-\mathrm{CWT})^{2}$ in separating the different spatial and temporal characteristics of the video into separate bands. The frequency information in each complex band is used to evaluate the quality of the distorted video sequence in a FR manner.

\subsection{Contributions}

In this thesis, we propose using the dual-tree wavelet transform with the CW-SSIM algorithm for image quality assessment. As there has not been a full evaluation test conducted to evaluate and compare the CW-SSIM algorithm with the PSNR and the SSIM algorithms, we perform a full evaluation test using the LIVE database subjectively score images.

\footnotetext{
${ }^{2}$ We use the notation of $[22]$ with $\mathbb{C}$ indicating complex to avoid confusion with the continuous wavelet transform (CWT).
} 
To eliminate the complexity associated with motion estimation in the current video extension of the SSIM algorithms, and to provide a simple to implement video assessment tool, we proposed using the 3-D DT-CWT with the CW-SSIM algorithm for video quality assessment. The proposed algorithm is able to account for temporal artifacts in the video sequence without the need for an explicit motion estimation step.

\subsection{Overview of Results}

The proposed DT-CWT implementation of the image $\mathbb{C W}$-SSIM algorithm was tested using the LIVE scored image database. The algorithm was compared against results from the PSNR test, the SSIM algorithm, and a steerable pyramid implementation of the $\mathbb{C W}$-SSIM algorithm. Our test results showed that the $\mathbb{C W}$-SSIM algorithm out performs both the PSNR and the original SSIM algorithm. Also, the performance evaluation shows that the dual-tree implementation of the CW-SSIM algorithm has the highest performance with higher prediction accuracy than the steerable pyramid implementation. The algorithms Pearson's correlation and the Spearman's rank order correlation coefficients with the subjective image scores were 0.9309 and 0.9378 , respectively.

Our proposed 3D-DT-CW-SSIM algorithm was evaluated against videos from the video qulaity experts group's (VQEG) multimedia (MM) project. The results were compared with those collected form the video-SSIM algorithm [19]. The 3D-CWSSIM algorithm has shown a higher performance when tested against low motion video sequences than that of the video-SSIM algorithm. The algorithm's overall performance was significantly higher than the video-SSIM algorithm when tested using all the available video sequences. 


\subsection{Organization of Thesis}

This thesis is organized in the following manner. Chapter 2 will give an in-depth background of image and video quality assessment, presenting and discussing a number of the available HVS based objective assessment algorithms. Chapter 3 presents the structural similarity index and its extension to the complex wavelet domain. The video implementation of the algorithm is discussed in the same chapter. Chapter 4 will review the wavelet transform and present the dual-tree complex wavelet transform. Chapter 5 and Chapter 6 will present the proposed image and video approaches, respectively, with their implementations and test results. Chapter 7 is the conclusion. 


\section{Chapter 2}

\section{Background}

\subsection{The Human Visual System (HVS)}

With human observers as the final "receiver" of the video, it was important for engineers to understand the functional process of the human visual system (HVS). Unfortunately, understanding the way information is collected by the human eye is not sufficient since much of visual perception happens in the brain. Defining the way captured information is being processed plays a major role in the development of image and video processing and quality prediction algorithms.

Testing HVS properties were conducted via physiological and psychophysical experiments [23-25]. Tests are generally conducted by presenting different stimulus to human subjects that in turn respond to the stimulus. The responses from the subjects are collected and analyzed to answer HVS processing questions.

Different characteristics of the HVS were identified and used in the development of many image and video processing and quality assessment algorithms. Although our work does not model the HVS, we present three important features of the HVS that are normally included in HVS based algorithms, which are (i) multi-channel structure, (ii) contrast sensitivity and (iii) the visual masking effect. 


\subsubsection{The multi-channel structure of the HVS}

Electro-physiological and psychophysical experiments conducted on the human primary visual cortex indicate that the neurons respond to stimulus frequencies in a bandpass way $[26,27]$. This means that the human brain processes the visual information by decomposing it into different channels. The visual decomposition channels, known as perceptual channels, are bands that are distinct in their spatial and temporal frequencies as well as their orientations. Further studies in the composition of the perceptual channels $[28,29]$ suggests that there are two temporal channels, transient and sustained. The transient channel is sensitive to moving patterns were as the sustained channel is sensitive to still or slow moving patterns. Also, the research indicated that there are between four to seven bands of spatial frequencies with between four and eight equal width orientation bands for each spatial band $[10,13]$. Different transforms were developed that simulate the HVS's channel decomposition that includes Watson's cortex transform [30] and the perceptual sub-band decomposition (PSD) by Senane et al. [31]. A typical perceptual channel decomposition is shown in Figure 2.1.

\subsubsection{The contrast sensitivity of the HVS}

The contrast sensitivity of the HVS refers to the response of the human's eye to the frequency of the perceived signal. The HVS can only detect a signal if its frequency is greater than a certain threshold known as the detection threshold. Since signals in the HVS are processed after being broken into the different perceptual channels, there is not a single threshold but a detection threshold for each perceptual channel. There has been much research tying the different frequencies with their detection threshold in what is known as the contrast sensitivity function (CSF) [33-35]. 


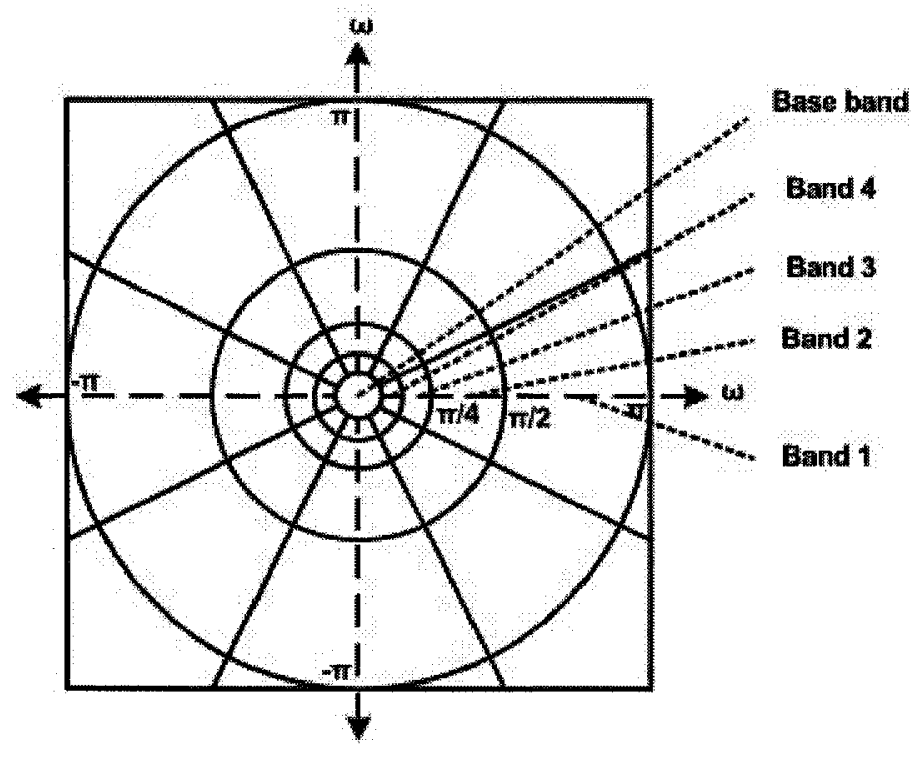

Figure 2.1: Typical HVS perceptual channel decomposition similar to that in [12] and [32].

\subsubsection{The masking effect in the HVS}

Visual information is very rich with stimuli. This raises the question of how does the HVS perceive multiple stimuli? And how do different stimuli affect each other perceptually?

Masking can be defined as the interaction between different stimuli and the effect of one stimulus on the perception of another in the same visual scene. Studies in the HVS indicate that both stimuli are seen, as long as they both exceed their detection threshold. However, a stimulus (masker) can change the detection threshold of another stimulus (maskee) making it harder on the HVS to detect it. Different algorithms were proposed to simulate the masking effect of the HVS, such as functions discussed in [35-37], and were used in quality assessment and compression algorithms.

Studies indicate that masking occurs between stimuli in different frequency channels, orientation channels, temporal channels (for video) and even color layers. However, masking is at its greatest when the two stimuli are approximately at the same 
location and have approximately the same frequency and orientation. For that, intrachanncl masking effects (between stimuli in the same perceptual channels) are studied, exploited and accounted for more than inter-channel masking effects.

Another type of masking, known as entropy masking, was first proposed and investigated by Watson et al. in [38]. Entropy masking refers to the masking effect occurring due to semi-local complexities of the image. It is known that the HVS is less likely to detect a stimuli/error that is located in a highly textured area (spatially) or high motion region (temporally). This masking effect was included in the quality assessment algorithms tested in [39]. It can be seen from their results that using entropy masking with intra-channel contrast masking can enhance the prediction performance of the quality assessment algorithms.

\subsection{Distortions that can occur in Digital Images and Videos}

Depending on the processing system producing the digital images and videos, different artifacts can become visible in the final product. In this section, we will briefly present some of the distortions that occur in digital images and videos due to processing. See Figure 2.2 for examples.

- Blocking Effect: also known as the checkerboard effect, is a very common artefact in block-based image and video coding algorithms, such as JPEG image compression and MPEG video compression systems. As the processed image/frame is broken into small (usually $8 \times 8$ ) blocks that are quantized independently, the individual blocks become visible in the decoded image as the bit rate is decreases. 
- Blur: blur normally refers to the loss of detail and edge information in the reconstructed image/video, making them appear out of focus. This artefact normally happens as high frequency information is lost due to processing. This effect can be seen when the high frequency bands are coarsely quantized in wavelet based compression algorithm.

- Color Bleeding: refers to color reflection/smearing between different colored object in the image. This effect is normally due to heavy quantization of chrominance information in colored images.

- Posterization: known as banding sometimes, is an effect were a number of smoothly transitioned color toned regions are grouped together and represented in a fewer number of color tones. The effect results in having areas with a smooth change in color represented by a number of color bands with a noticeable step between them. This effect occurs primarily when the depth of color used is reduced in the processing algorithm (for example moving from a $24 \mathrm{bit} / \mathrm{pixel}$ to $8 \mathrm{bit} / \mathrm{pixel}$ image).

- Ringing Effect: the ringing effect (known also as Gibbs' phenomenon) occurs due to, among others, the quantizing of individual coefficients. Ringing is visible as a number of high frequency irregularities, that look like rings or waves, around edges in the reconstructed image or video frame (see [40] for a more thorough discussion).

- Mosquito Noise Effect: this effect can be seen as the temporal version of the ringing effect. Mosquito noise effect is seen as high frequency flickering around edges in a video sequence. Similar to the ringing effect, mosquito noise effect can be a result of quantization. 
- Ghosting: as a result of quantization errors in video frames, a video decoder sometimes fails to decode part of the frame sequence. As more information is received, the decoder keeps updating the sequence by building on the error information resulting in a ghost image. This effect normally happens when video errors occur in videos that use I-frames (reference frames) and P- and B-frames (frames predicted from the I-frame). The effect normally stays visible until the next I-frame is received.

- Random Noise: random noise, white noise, and salt and pepper noise are random errors in the processed signal's coefficients that result in adding positive or negative high frequency changes in the coefficient's value. This effect can be visible as the affected pixel's values becomes very different than its surrounding and shows up as a dot on the reconstructed image/frame.

- Packet Loss: packet loss is not an artefact per se, but a cause of an artefact. As packets containing image/video information get lost in noisy transmission channels, different artefacts can be visible in the final reconstructed image/video. The type of artefact visible depends on many factors that include: the amount of lost information, the importance of the lost information, the compression algorithm used, and the coding technique used in coding the packets.

\subsection{Subjective Quality Assessment}

Since humans are generally the final receiver of image and video signals, subjective testing is considered to be the benchmark when evaluating the signal's quality. Subjective tests use humans as the judges in evaluating images and videos, generally in a well defined and controlled environment. The test subjects are presented with the 


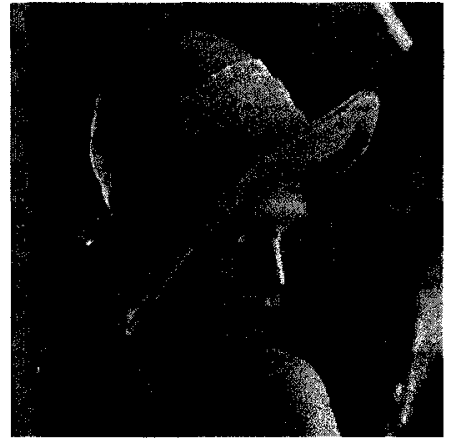

(a)

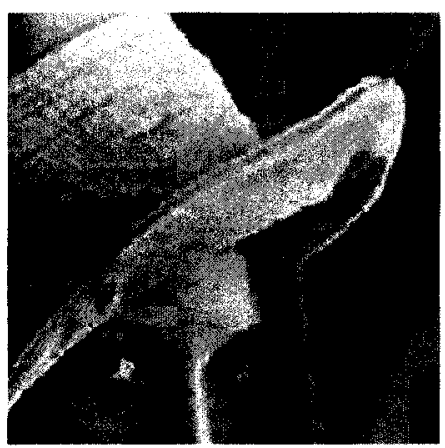

(d)

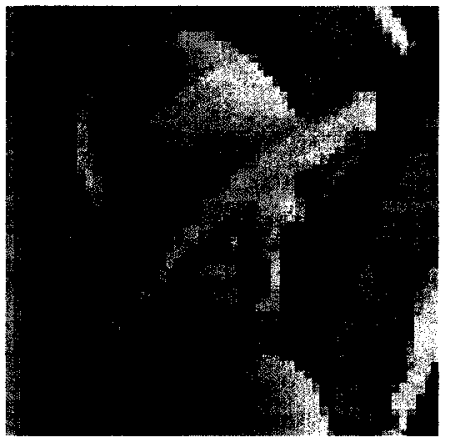

(b)

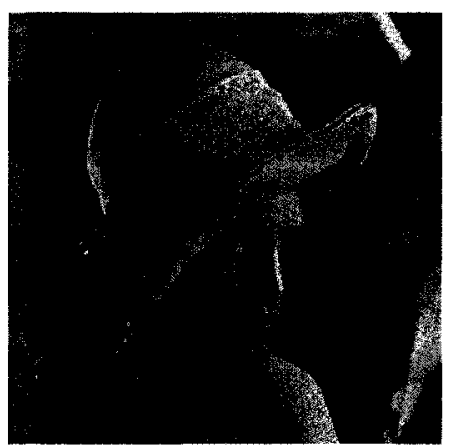

(e)

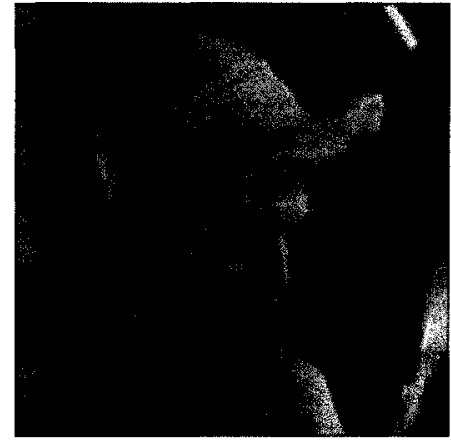

(c)

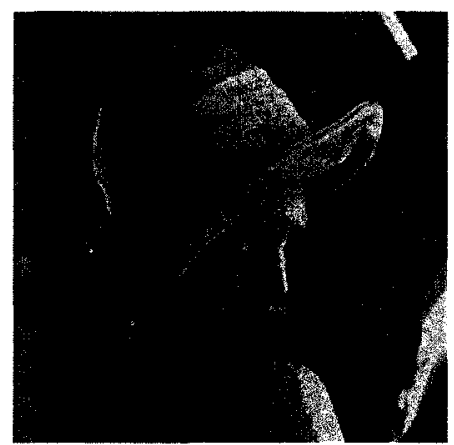

(f)

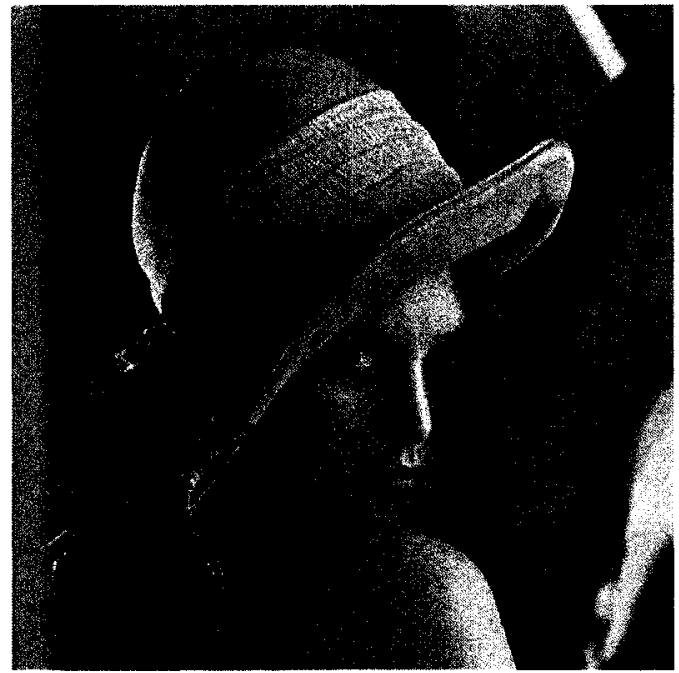

(g)

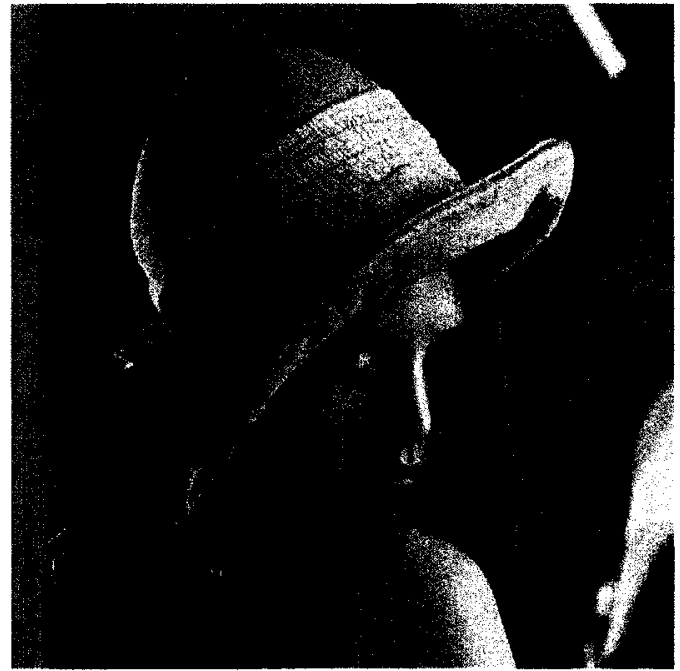

(h)

Figure 2.2: Examples of different distortions that can occur in Digital Images and Videos. (a) Lena's gray scale reference image, (b) blocking artefact, (c) blur, (d) ringing effect (image was cropped to better show the effect around the eyes and the hat), (e) random noise in the form of white Gaussian noise, (f) posterization, (i) Lena's color reference image, (j) color bleeding. All images original images are $512 \times 5128 \mathrm{bits} /$ pixel images 


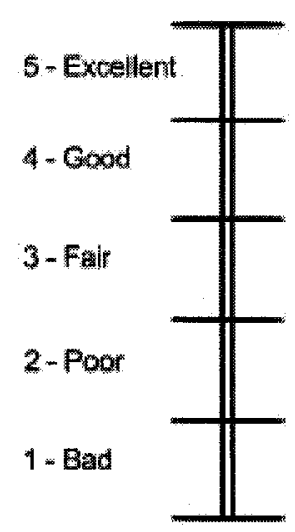

(a)

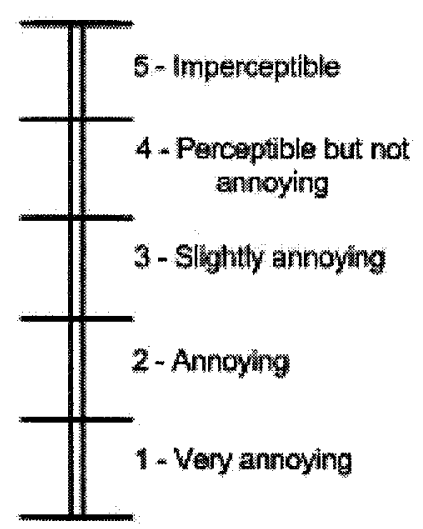

(b)

Figure 2.3: Generalized grading scale sheets used with subjective tests: (a) used for quality assessment, and (b) used when assessing the effect of a perceptual impairment.

test material in a pre-defined manner and are asked to give their opinion in a scale format. The subjects are normally screened before allowed participation in the test for visual acuity. Also, subjects are generally chosen to represent a wide range of viewers to insure score reliability. Nonetheless, some tests can limit participation to subjects who are considered to be experts in the field. This requirement is imposed sometimes when trying to look for a specific impairment in the test image or video sequence.

Subjective test grading scores are normally recorded using a scale from 1 to 5 (1 is the lowest perceived quality and 5 is the highest). The subjects are provided with sheets containing the scoring scale and are asked to mark the appropriate quality for the test material. Example scoring sheets are presented in Figure 2.3. The scores collected from all subjects are averaged to form the mean opinion score (MOS) values from which the difference mean opinion scores (DMOS) are often generated. DMOS values are generated by taking the difference between the MOS of the test image/video and that of its reference, to generate reliable FR quality results. 


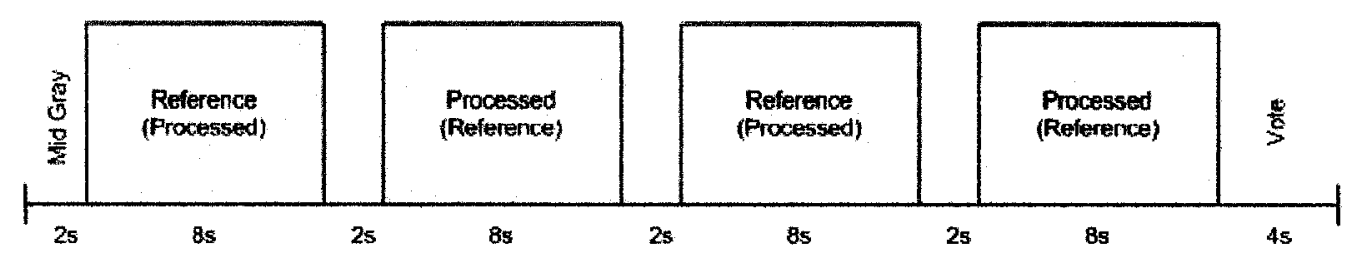

Figure 2.4: DSCQS basic test cell similar to that used in [41].

The main difference between subjective tests is the method used in presenting the test material to the subjects. Different methods can be defined and used, based on the targeted goal behind the test, that can be categorized as single stimulus (SS) or double stimulus (DS) tests. SS subjective tests present only a single test image/video to the subject at a time, who is then asked to score it. This category of testing is used to generate scores based on the general experience of the viewer, since viewers do not have access to the reference material. DS subjective tests present both the reference and test material to the test subjects, as a pair, who are asked then to provide a quality scores. Subjective scores collected using DS tests represent the quality of the test image/video in relation to its reference rendering them more suited in evaluating perceived differences. For that reason, DS test scores are what's normally used as the benchmarks when evaluating full reference objective quality assessment algorithms.

The Video Quality Expert Group (VQEG) used a DS subjective test approach to generate the subjective scores of all of their 8 seconds long video sequences. The DS test used by VQEG is the double stimulus continues quality scale (DSCQS) method [41]. DSCQS was used as it has shown to have low sensitivity to contextual effects. To assess videos using the DSCQS, subjects were presented with a pair of sequences two consecutive times. The order in which the reference and test sequences were displayed was chosen at random for each pair. Two seconds were given between sequences with 4 seconds at the end for voting/scoring. Figure 2.4 illustrates a basic cell used in the DSCQS method similar to that used in VQEG's video scoring process. 


\subsection{Objective Quality Assessment}

Subjective tests, although producing the most reliable quality scores, are not desired generally when evaluating processing algorithm. This lack of desire for subjective tests is due to their preparation time, running time, cost, and their environment conditions/control requirements. The desire to step way and avoid subjective test has been the main reason motivating the development of objective quality prediction algorithms for both images and video sequences.

Objective tests do not only get over subjective test's requirements, but when it comes to image and video processing, they present new advantages that are highly desired, such as [19]:

- they can be used as a quality control system to monitor the quality of images/videos;

- they can be used to benchmark different image/video processing systems; and

- if embedded with the processing system, they can be used for system optimization.

Objective algorithms can be divided into three categories based on the availability of the reference material: FR, RR, and NR algorithms. FR assessment requires the reference data to be available. It relies on comparing the reference data to the test data (generated by processing the reference image or video) in a component by component basis. The RR assessment algorithms uses data/information extracted from the reference material to test the quality of the test data. RR algorithms are very useful when the reference data is not available in its entirety or if it cannot be present with the test data due to some constraints (i.e., storage capacity or transmission limitation/bandwidth). Finally, NR assessment algorithms estimate the quality 


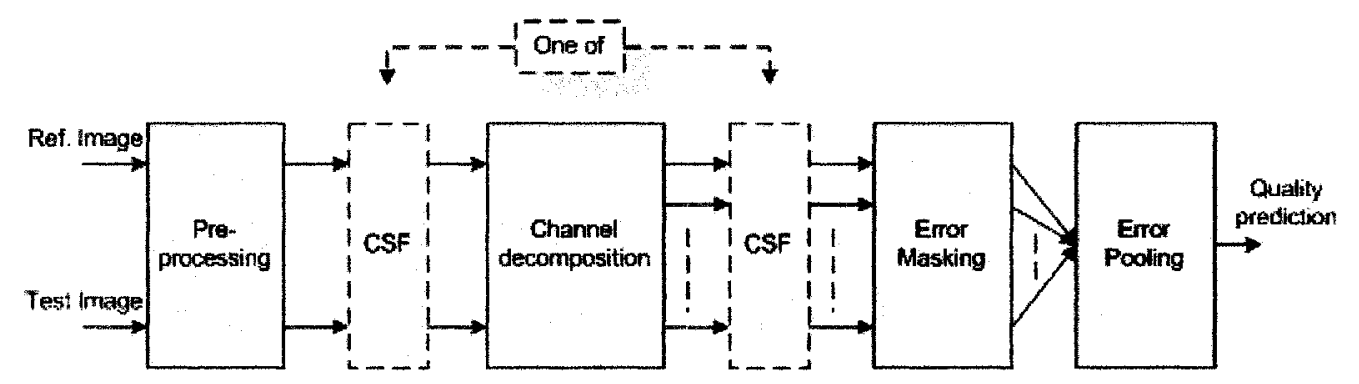

Figure 2.5: Block diagram for a general perception-based objective assessment algorithm. This diagram based on that in [42]

of the test data without having any information about the reference data. NR algorithms, which are generally considered to be the ultimate gaol of objective assessment algorithms, are highly useful as they can be use in cases where the reference data is completely unavailable. However, they are very complicated to design and to the best of our knowledge there is not any broadly applicable NR algorithms that work well on a broad range of distortions.

In this document, our focus will be image and video quality assessment algorithms that fall under the FR category.

\subsection{Perceptual Quality Assessment - General Framework}

The goal of perceptual objective assessment algorithms is to provide a quality prediction of some media, being an image or a video sequence, based on the characteristics of the HVS. Many different algorithms are available for quality assessment that vary in their implementation (see Sec. 2.6 and Sec. 2.7). However, a general framework can be derived from studying the different HVS based assessment algorithms. Figure 2.5 illustrates the basic blocks comprising a general HVS based quality assessment 
algorithm. The individual blocks are discussed below.

- Pre-processing: this pre-processing step is done to prepare the test media for the rest of the algorithm, based on the criteria of that algorithm. This step might include media alignment, color transformation to a suitable color space, light adaptation [36], perception non-linearities function [43] and/or point spread filtering to simulate the eye's optics [44].

- CSF: to account for the variation in the HVS's sensitivity to different frequencies, a CSF is generally applied to the media at one of two points; before the channel decomposition, as in [36], through the use of filters to simulate the CSF's response, or after the channel decomposition, as in [43] for normalization before applying masking [19]. Some algorithms do not have a CSF step. However, it is accounted for when applying masking [12] or while designing the channel decomposition filters, as in the Sarnoff's just noticeable difference algorithm.

- Channel Decomposition: visual cortex electro-physiological and psychophysical experiments indicate that the neurons in the HVS respond in a bandpass fashion to stimulus frequencies [26,27]. This finding lead to many approaches that simulate the decomposition of media into different perceptual channels for processing. Channel decomposition can be highly beneficial, as the HVS responds with different sensitivities to stimuli with different frequencies and orientations.

Different methods have been used for test media decomposition into channels that simulate the HVS's perceptual channels, that includes: the cortex transform, as in [36]; Laplacian pyramid and steerable transforms, as used in [43]; and Gabor filters, as in [45]. 
Simpler transforms have been used for image and video decomposition such as: the discrete cosine transform (DCT), as used by Watson in $[25,46]$; and more recently discrete wavelet transforms (DWT), used in [11, 12] and [47]. Such transforms have been used due to their suitability for certain image and video processing applications rather than their accuracy in simulating the perceptual channel decomposition [42].

- Masking: errors in each perceptual channel are masked by adjusting the base sensitivity of the channel and normalizing the errors, masking them based on the presence of other stimuli in that channel. This is done as the HVS's sensitivity to each component in each perceptual channel changes depending on the presence of other stimuli. Depending on the algorithm's design, intra- and/or interchannel masking effects may be considered.

- Error Pooling: this final step of the algorithm combines the different error values from different perceptual channels into a single measure of quality or an error map. Generally, Minkowski's distance $\left(L_{p}\right.$-norm of the difference image/sequence) is used for this step. Error pooling can be performed multiple times in an algorithm to generate more intermediate quality results. The multistage perceptual quality assessment (MPQA) algorithm [48] (Sec. 2.6.10), which uses an initial pooling to generate an error map followed by a second pooling to generate the quality metric, is an example of multi-stage pooling.

\subsection{Image Quality Assessment}

In this section, we will present some of the work that has been done in the field of image quality assessment. 


\subsubsection{Element based algorithms}

This set of algorithms works by comparing the picture's components on an element by element basis defining the error between each two. Such element based algorithms include the mean squared error (MSE) and peak signal-to-noise ratio (PSNR). The MSE and PSNR are well defined and apply directly to grayscale images. However, no standard is set when they are applied to colored images. The MSE is simply the average squared difference between the two pictures' elements in a direct fashion. The MSE is defined for two grayscale images $I$ and $\hat{I}$ by

$$
\operatorname{MSE}(I, \hat{I})=\frac{1}{X Y} \sum_{x=1}^{X} \sum_{y=1}^{Y}[I(x, y)-\hat{I}(x, y)]^{2}
$$

where $X$ and $Y$ are the dimensions of each of the two images.

The PSNR is the ratio between the maximum power the image can take to the amount of error in that image. The PSNR is related to the MSE and is defined in decibels as

$$
\operatorname{PSNR}(I, \hat{I})=10 \log _{10} \frac{m^{2}}{M S E(I, \hat{I})}
$$

where $m$ is the maximum value the pixel can take. For example, for an 8-bit per pixel image, $m=255$.

MSE and PSNR are widely used, due to their clear physical meaning and calculation simplicity, in evaluating both image and video signals. However, they do not correlate well with subjective scores $[3-6,17,48]$. Recent work in the area of video quality assessment shows that with the use of perceptual weights, the correlation of PSNR results with subjective scores can be increased [49]. 


\subsubsection{Fourier magnitude difference}

The Fourier magnitude difference is an early algorithm presented and discussed by Mannos and Sakrison in [43]. Mannos and Sakrison recognized the value of Shannon's rate-distortion function, which compares the rate of encoding/transmission vs. the distortion in a signal. Also, they realized that for rate-distortion to be usable with images, an algorithm to evaluate the distortion in an image that correlates with subjective results is needed.

The algorithm discussed in [43] was adapted and evaluated by [50], who worked on normalized grayscale images, as one of three perceptual quality algorithms. As the HVS is more tuned to detecting the luminance variation as opposed to the absolute luminance, the algorithm starts by normalizing the luminance in the images with the mean luminance value $l_{m}$ of each image. To account for the perception nonlinearities, Mannos and Sakrison found it suitable to apply $f(\dot{X})=\dot{X}^{0.333}$, where $\dot{X}$ is the luminance normalized image $\left(\dot{X}=\frac{X}{l_{m}}\right)$. After this initial step, the resulting images are transformed using the fast Fourier transform (FFT) and the resulting coefficients are filtered by a function $A(r)\left(r=\sqrt{u^{2}+v^{2}}, u\right.$ and $v$ are the horizontal and vertical spatial frequency in cycles per degree) simulating the contrast sensitivity of the HVS.

The output of an image $X$ from the algorithm can be expressed as

$$
G=\mathfrak{F}\left(\left(\frac{X}{l_{m}}\right)^{0.333}\right) A(r)
$$

where $\mathfrak{F}$ is the FFT and $A(r)$ is defined as

$$
A(r)=2.6[0.0192+0.114 r] \exp \left[-0.114 r^{1.1}\right]
$$


The distortion between the two images $X$ and $Y$ ( $Y$ is the distorted version of image $X$ ) is taken as the MSE of $G_{X}$ and $G_{Y}$ making it a measure in the difference of the magnitudes of the Fourier transforms.

\subsubsection{Magnitude and phase model based on Gervais et al.}

The magnitude and phase model, which is based on a proposal by Gervais et al. in [51], is different than the model by Mannos and Sakrison (discussed in Sec. 2.6.2) mainly by its use of the phase information of the FFT along with the magnitude information.

The algorithm starts by normalizing the image using its mean luminance $l_{m}$. The FFT is performed on the normalized image producing the magnitude set $m$ and the phase set $p$. The magnitude information is filtered using an anisotropic filter function producing the values $G$. The anisotropic filter used was the one presented by Campbell et al. in [23].

The quality index of the distorted image $Y$ is taken as the distance between it and the reference image $X$ using

$$
\operatorname{Dist}(X, Y)=\frac{1}{N} \sum\left\{\left[\left(\log G_{X}+1\right)-\left(\log G_{Y}+1\right)\right]\left(1+p_{X}-p_{Y}\right)\right\}^{2}
$$

where $N$ is the total number of coefficients from the $\mathrm{FFT}^{\prime}$.

\subsubsection{Visual difference predictor}

Proposed by Scott Daly in [36], the visual difference predictor (VDP) is a well known distortion metric that accounts for HVS characteristics such as contrast sensitivity and masking. 

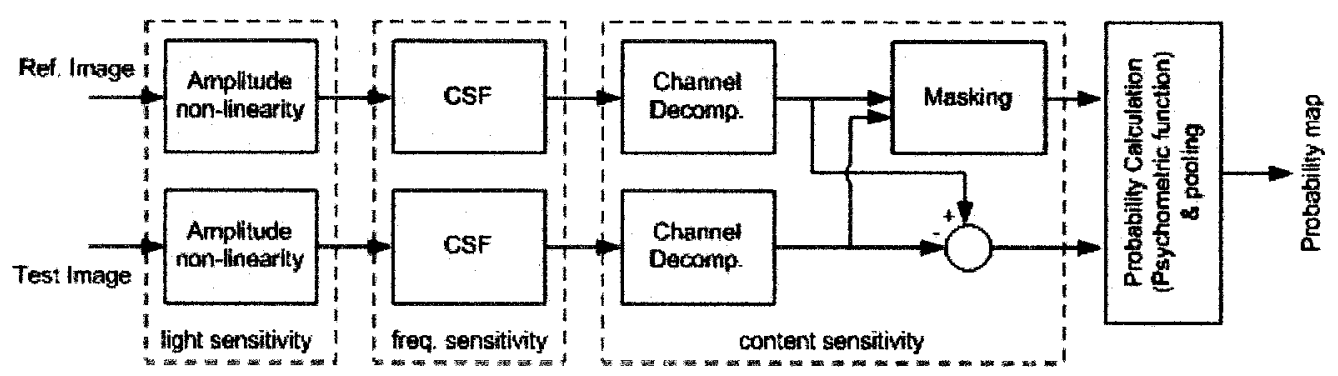

Figure 2.6: Block diagram representation of the VDP highlighting the three main HVS simulation stages.

The algorithm takes in two images and outputs an error detection probability map representing the probability of error detection at each image location. In simulating the HVS, the algorithm passes the images through three major steps: light sensitivity, frequency sensitivity, and content sensitivity. See Figure 2.6 for a block diagram representation of the VDP algorithm.

The light sensitivity, which refers to the amplitude non-linearity response of the HVS, is accounted for by transforming the image using a simplified retinal response function, transforming the input luminance image $X$ to $\dot{X}$. The transform is done using

$$
\dot{X}=l_{i j} /\left(l_{i j}+12.6 l_{i j}^{0.63}\right)
$$

where $l_{i j}$ represent the luminance value at pixel location $i, j$.

The second block, the frequency sensitivity, is simulated by calculating the FFT of the amplitude non-linearity image $\dot{X}$ and then filtering the resulting magnitudes by the CSF $A(r)$, where

$$
A(r)=\left(\frac{0.008}{r^{1.5}+1}\right)^{-0.2} 1.24 \sqrt{r} \exp (0.3 \sqrt{r}) \sqrt{1+0.06 \exp (0.3 \sqrt{r})}
$$

with $r=\sqrt{u^{2}+v^{2}}$ and $u$ and $v$ are the horizontal and vertical spatial frequencies. 
The final HVS simulation block, accounting for the content in the image, is the most complicated of the blocks. The content sensitivity is accounted for by applying contrast masking which requires perceptual channel decomposition. The perceptual decomposition is performed using a cortex transform similar to the one proposed by Watson [30] and masking is applied on a channel-by-channel and pixel-by-pixel basis.

After masking has been applied to all the channels, the detection probability is calculated for each pixel using a psychometric function, resulting in a detection probability map for each channel. The probability maps from all the channels are combined to generate one map for the entire image.

\subsubsection{Model after Daly}

An implementation of the VDP discussed in Sec. 2.6.4 was modeled by [50]. In their implementation, the images are first transformed to account for non-linearity using the retinal response function (2.6). The output of the retinal response function is transformed to the frequency domain using the FFT. An output $G$ is obtained for each of the reference and test images by applying the CSF expressed in (2.7) on the magnitudes from the FFT. The distance between the reference image $X$ and the test image $Y$ is taken as the MSE of $G_{X}$ and $G_{Y}$.

This implementation, which differs from the VDP in that it does not apply contrast masking nor does it build a detection probability map, was tested and compared by [50] along with the implementations discussed in Sec. 2.6.2 and Sec. 2.6.3. The model after Daly's VDP showed better correlation with subjective scores than the other two tested algorithms. 


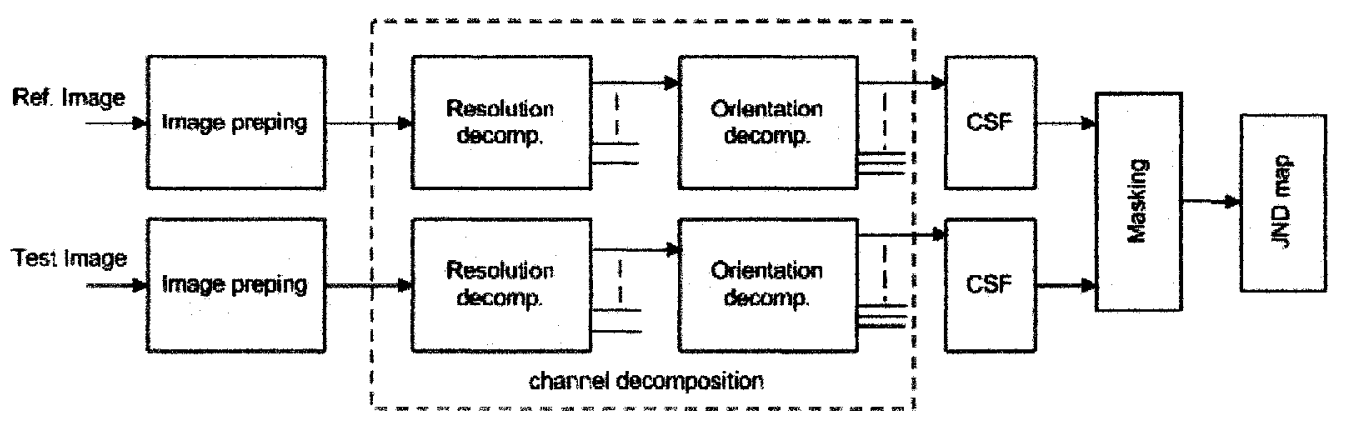

Figure 2.7: Block diagram representation of the VDM.

\subsubsection{Visual discrimination model}

The visual discrimination model (VDM) operates in the spatial domain, unlike Daly's VDP which operates in the FFT domain. The VDM starts off by preparing the image through convolving it with a point spread function, which simulates the optics in the human eye, and then re-sampled to reflect the sampling of the retina's receptors.

Once the preparation step is complete, the image is decomposed into different frequency and orientation channels. The decomposition is achieved by separating the image into different resolution bands using a Laplacian pyramid followed by orientation decomposition of each band into different channels. The orientation decomposition is done through steerable filters as discussed by Freeman and Adelson [52].

A CSF is applied to each channel followed by a masking step which is implemented using a sigmoid non-linearity function. A just noticeable difference map is generated from the masked responses and used to calculated the distance between the image and its reference as a Minkowski sum ( $L_{p}$-norm). Figure 2.7 shows a simple block diagram of the VDM algorithm. 


\subsubsection{Gabor pyramid visible difference predictor}

Inspired by the the VDP [36] (Sec. 2.6.4), another VDP algorithm was proposed in [45]. Taylor et al. proposed a VDP algorithm that uses the Gabor pyramids to decompose the image into the perceptual channels. The selection of the Gabor filters is due to their success in simulating the perceptual channels in the visual cortex. Also, this transform was chosen as it was successfully used in predicting image sharpness [53] among other applications.

The algorithm, illustrated in Figure 2.8, takes two luminance images and performs a Gabor pyramid channel decomposition on them. The channel decomposition is accomplished in two steps; resolution pyramid step and orientation channel step. The resolution pyramid is done through iteratively using a low-pass filtering and decimation. Gabor decomposition is applied to the base images (a base image is the output of one of the pyramids resolution levels) to get different orientation channel images. [45] uses eight Gabor decomposition channels, each tuned to a different orientation.

The parameters for the Gabor wavelets were determined using psychophysical experiments. Also, a look up table (LUT) was constructed to contain the psychometric functions that will be used in generating the visible distortion maps. To generate the distortion maps, information from the base image, the channel images, and the masked error image of each channel is used to select the appropriate psychometric function to be used for that channel. The final VDP map of the image is built through probability summation of all the channels' prediction probability maps.

\subsubsection{Wavelet image quality measure}

The HVS is sensitive to the frequency changes in its visual cortex's different bandlimited visual channels which have been successfully simulated by a set of Gabor 


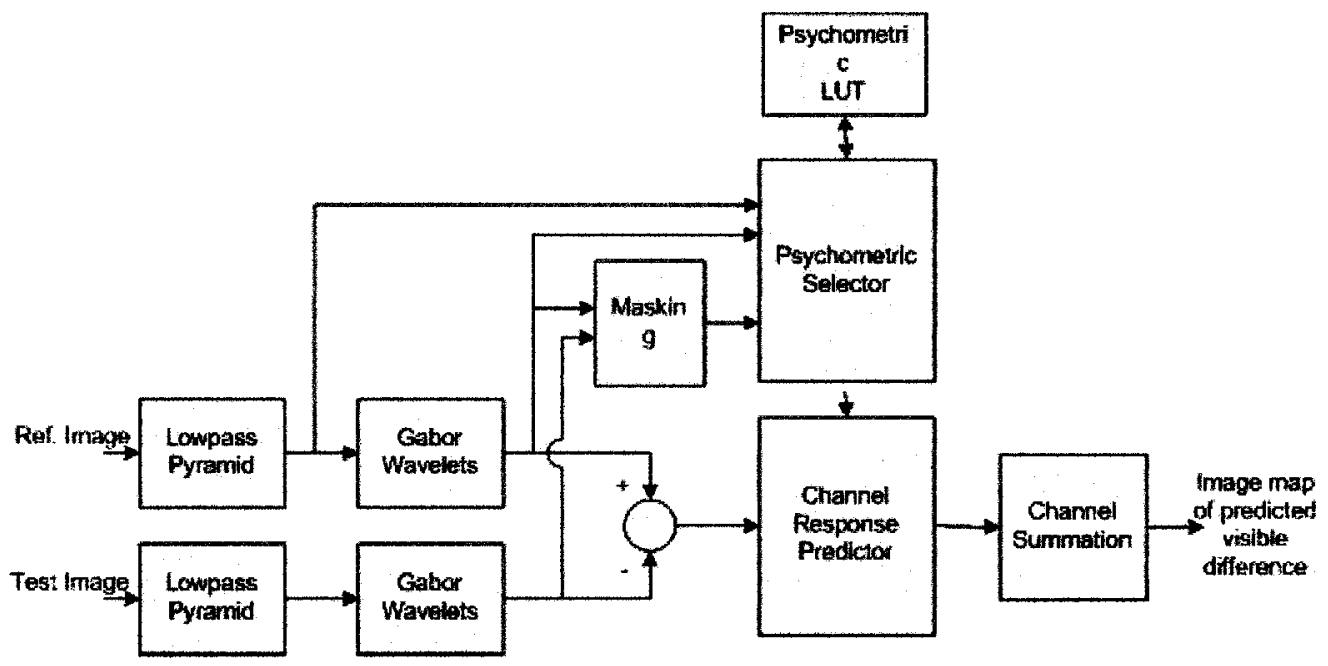

Figure 2.8: Block diagram representation of the Gabor pyramid VDP as in [45].

filters. However, although Gabor filters have been deemed to simulate the HVS's visual channels well $[9,10,45,53]$, selecting the Gaussian parameters poses a challenge when trying to obtain the best filter set.

As an alternative, [11] proposed the use of the wavelet transform to transform the image to the frequency-like domain as the means for perceptual channel decomposition. The wavelet transform was selected due to its multi-level frequency and orientation decomposition, ability to perform an inverse transform, and it's increased use in image and video coding over the use of Gabor filters. In [11], both the reference and distorted images are transformed using a Haar wavelet transform and a suprathreshold is derived form the contrast information from each channel. The threshold is modified based on information in neighboring channels to account for the inter-channel threshold effect. Also, the suprathreshold is modified to account for inter-channel masking as the perceptual channels are not completely independent. Once the suprathreshold values have been calculated, it is used to obtain the perceptual distortion as a weighted sum of the frequency mismatch of each transform band and the total distortion is the sum of all the band's distortions. 
Predicting the quality of images using the DWT perceptual channel decomposition was evaluated recently be Ninnassi et al. in [39]. The evaluation indicated that using DWT in quality assessment algorithms is worth the little reduction in the algorithm's prediction performance, as DWT reduces the decomposition computation time and complexity.

\subsubsection{Wavelet visible difference predictor}

Similar to [11], [12] recognized the value of working in the wavelet domain and proposed a wavelet visible difference predictor to estimate the amount of visible error in images. The algorithm, which was also based on the VDP algorithm [36] (Sec. 2.6.4), works by transferring both images, the reference and the test images, to the wavelet domain. The amount of tolerable noise is calculated using the error signal and wavelet based contrast masking. This information is used to estimate the detection probability for each coefficient which in turn is combined to obtain the error detection probability at each pixel. This algorithm does not model the CSF. However, the CSF is accounted for when estimating the wavelet contrast masking threshold.

\subsubsection{Multistage perceptual quality assessment}

Oh et al. [48] proposed an extension to the VDP algorithm [36] (Sec. 2.6.4), the multistage perceptual quality assessment (MPQA), and used it to evaluate the quality of lossy compressed X-ray cardio-angiogram images [48]. The algorithm, illustrated in Figure 2.9, uses the same amplitude non-linearity function and cortex transform used in the original VDP algorithm. However, this algorithm uses CSF and background uncertainty information based masking.

As the HVS is more tolerant to errors in highly textured areas compared to smooth 


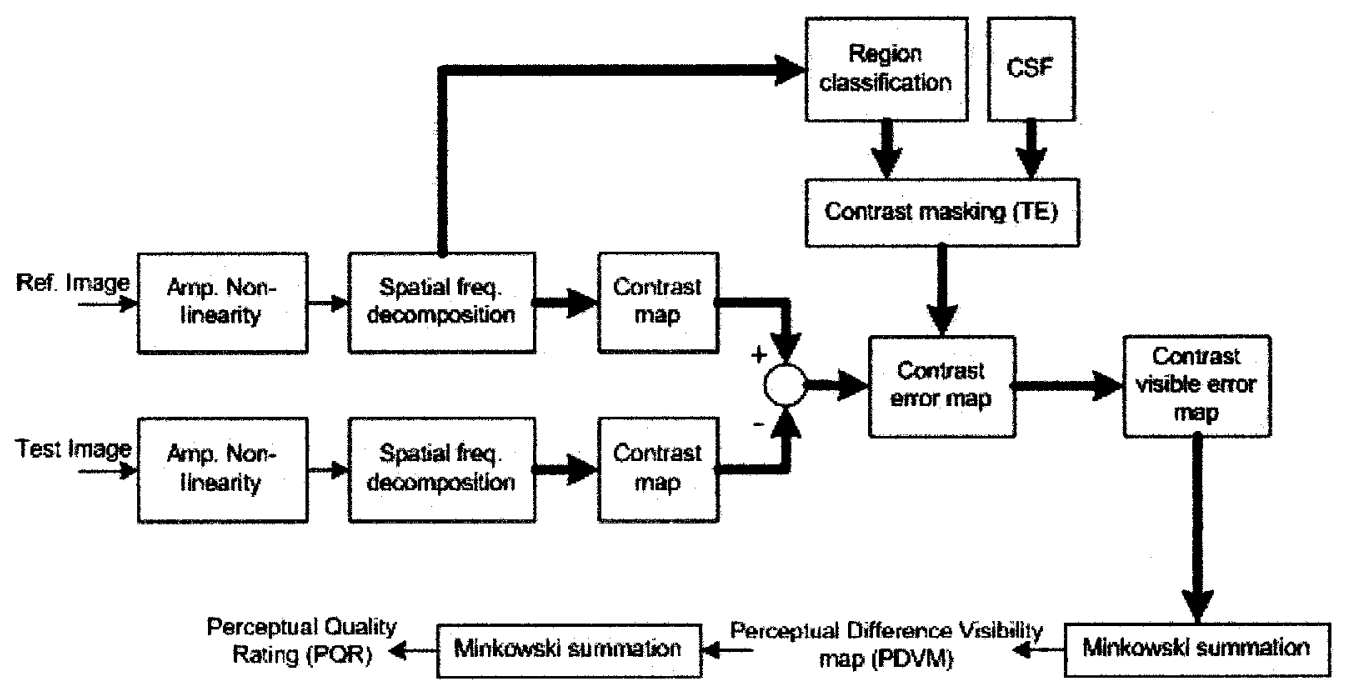

Figure 2.9: Block diagram representation of the multistage perceptual quality assessment algorithm as in [48].

areas, the contrast masking used in the MPQA algorithm adjusts the detection threshold to reflect the region the noise is in. This required the algorithm to run a region classification process before masking is applied. The regions in the decomposed image are classified as flat, edge or texture regions. Flat regions were selected as the areas where all the contrast values are less than the minimum threshold given by the CSF. Edge regions were identified using a Sobel edge detector. Areas that did not fit the criteria of the first two classification were considered texture regions.

Channel contrast error visibility maps are generated by masking the contrast error maps (the difference between a reference image's channel and test image's channel) which are used to generate the perceptual visibility difference map (PVDM). A single perception quality rating (PQR) value is calculated from the PVDM through a Minkowski error summation step. 


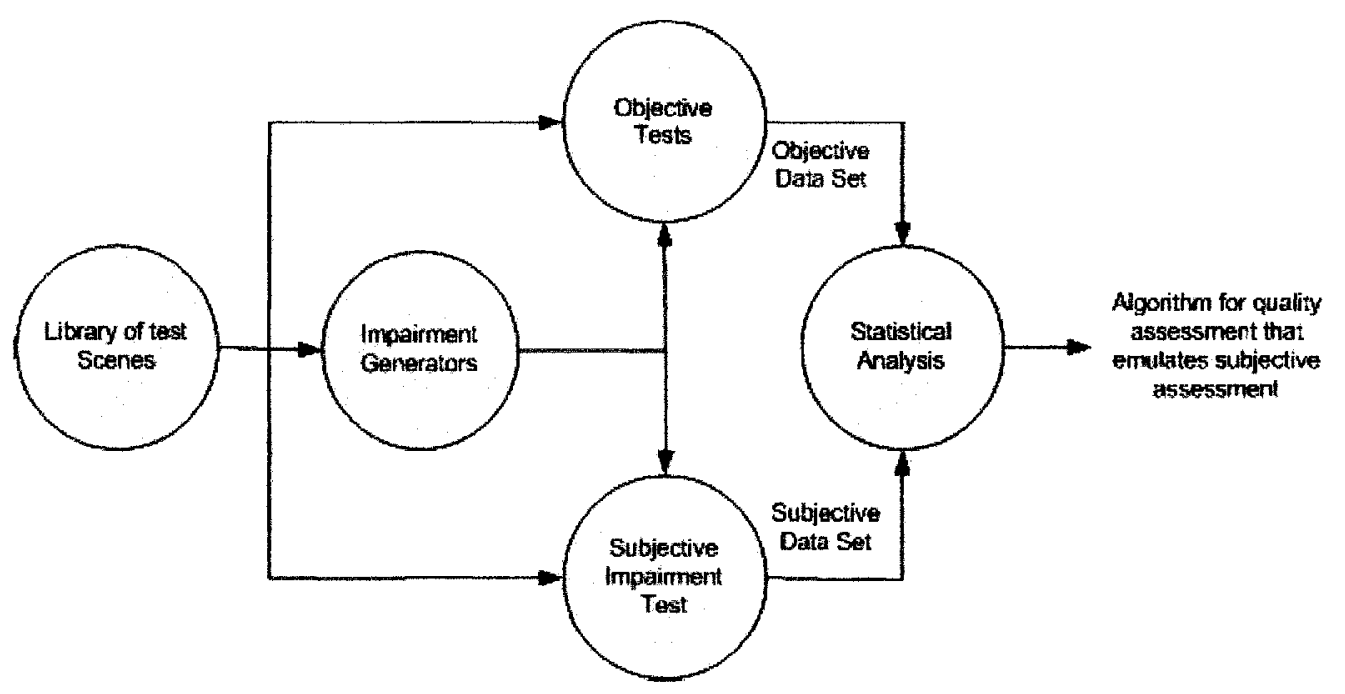

Figure 2.10: Voran's objective quality algorithm derivation process, based on that illustrated in [7].

\subsection{Video Quality Assessment}

Any image quality prediction algorithm can be used for video quality assessment on a frame by frame basis. However, such implementations are not desired as it does not account for motion or temporal artefacts. In this section, we will present some of the algorithms designed for perceptually assessing and predicting video quality.

\subsubsection{Emulating human perception with objective video quality measures}

Stephen Voran, in [7], discusses a methodology used to derive an objective test algorithm by emulating the subjective test results. The method, illustrated in Figure 2.10, operates on digital video in a FR manner. Initially, a collection of test video sequences is collected that includes a set of reference videos and a number of distorted version for each reference. The videos are scored subjectively under specific test conditions which are discussed in [7]. 
The objective testing part of the process works on the sequences on a frameby-frame basis. Every pair of video sequences (a distorted video and its reference sequence) undergoes a spatial and temporal alignment step before conducting any objective quality tests. As there could be frame repetitions in the distorted video, the temporal alignment works on a frame by frame bases, matching each frame from the distorted video with the closest frame to it, the frame with the smallest squared pixel error, in the reference video sequence.

It is known that the human visual system has a higher resolving power with still scenes as apposed to moving scenes. Therefore, before the objective tests begin, still and motion pixels are identified and separated. To identify the different pixels in frame $f_{k}$, the absolute difference frame $\left|f_{k+1}-f_{k-1}\right|$ is calculated first and a threshold is applied to the difference frame. All pixels with values smaller than the threshold are considered still pixels and the rest are motion pixels.

After the preprocessing step, a number of different objective tests are independently run to predict the quality of the test sequence. The objective tests selected have to meet certain requirements that include being functionally independent, stable, and have good computational efficiency. Voran suggested the use of and evaluated his approach using the quality tests outlined in [54].

Once the subjective and the objective test results are collected, a correlation analysis is carried out to determine the subset of the objective test combination that yields the best quality prediction algorithm.

The algorithm was tested on a small set of video sequences and showed high correlation results with subjective scores. Also, similar tests to those conducted in [7] were performed in [8], using only three measures for the objective tests that makes use of spatial and temporal information extracted form the sequence's frames. This smaller subset of objective tests used in [8] was chosen in an attempt to make Voran's 
algorithm more efficient and executable in real-time.

\subsubsection{The moving pictures quality metric}

The algorithm which is known as the moving pictures quality metric (MPQM) $[9,10]$ uses a spatio-temporal model of the HVS. As Gabor filter banks have a profile close to that of the perceptual channels, they were used in this multi-channel approach to simulate the perceptual channels of the HVS.

The algorithm uses a spatio-temporal contrast sensitivity function and intrachannel contrast masking to evaluate the amount of response the visual neurons have to the errors in the distorted sequence. Masking is done by taking the error sequence with the original sequence as a masker and going pixel-by-pixel and channel-by-channel to adjust the contrast detection threshold. The corrected threshold values are used with the error signal to generate just noticeable difference (JND) maps.

Since the human viewer does not process the entire scene as one unit, the signal is divided into 3D blocks with varied sizes; temporally based on the image's persistence on the retina and spatially to cover 2 degrees of visual angle. Probability summation is used to calculate the quality of each of the 3D blocks using the JND information. The global quality is obtained by averaging all the blocks qualities.

Due to its spatio-temporal filtering design, complexity, and memory requirement, the algorithm is slow and not very efficient in calculating the quality of large video sequences. Lindh and Lambrecht in [55] proposed the normalization video fidelity metric (NVFM) as an enhancement to the MPQM. NVFM was built as an extension of the normalization model by Teo and Heeger [56]. The algorithm uses steerable pyramid transform instead of Gabor filters and accounts for the normalization of the cortical receptive field responses. The new algorithm showed to be more efficient and 
gave better quality prediction than its original version.

\subsubsection{Sarnoff's just noticeable difference (JND)}

The Sarnoff just noticeable difference (JND) algorithm was introduced by Lubin and his colleagues at the Sarnoff corporation. Sarnoff's JND was considered by many a de-facto standard after commercializing its application and the introduction of Tektronics picture quality assessment instruments [13].

The JND algorithms operates in the opponent color space of Luminance (B-W) and colors (R-G) and (B-Y). Both reference and test video sequences are transformed from their input color space to cone long-medium-short (LMS) responses which in turn are converted to the opponent color space coordinates.

Once videos are pre-processed and are in the correct color space, a spatio-temporal decomposition is performed to simulate the HVS's perceptual channels. The decomposition, which results in two temporal channels with seven frequency resolution and four orientations each, is achieved by first applying a temporal decomposition using the two filters expressed in [13] as

$$
\begin{aligned}
& h_{1}(t)=a \exp (-a t) u(t) \\
& h_{2}(t)=b\left[\left(b t^{3} / 3 !\right)-\left(b t^{5} / 5 !\right)\right] \exp (-b t) u(t)
\end{aligned}
$$

where $u(t)$ is a unit step and both $a$ and $b$ are constants calibrated to fit the HVS's CSF.

A spatial decomposition follows the temporal decomposition and is computed using a Gaussian pyramid transform followed by a Laplacian pyramid. This is done to simulate local contrast computing while obtaining the different spatial resolutions. 
Freeman and Adelson's steerable filters [52] are used to obtain the four orientation channels. The CSF is not applied directly to the video sequences, since all the filters used in the spatio-temporal channel decomposition of the JND algorithm are calibrated to simulate the HVS contrast sensitivity.

Both intra- and inter-channel masking is accounted for in the JND algorithm. First, the coefficient values of each channel are squared and pooled then normalized by the squared detection threshold. After that, the contrast gain is controlled by dividing each filter's output by outputs from other filters in different temporal, resolution or orientation channels, accounting for inter-channel effects. This is followed by a point non-linearity function to account for the dips in the contrast curve. The final sequence's difference values are obtained as the $l_{2}$-norm of the difference sequence (difference between the reference and test sequence channels).

\subsubsection{Watson's digital video quality metric}

Andrew B. Watson in [57] proposed a video fidelity metric, dubbed the digital video quality (DVQ) metric. Unlike other algorithms discussed, Watson's DVQ works in the discrete cosine transform (DCT) domain.

The DVQ metric starts with a preprocessing step that may include cropping and registration. Also, before the algorithm starts, the video sequences are converted from their color space to the $\mathrm{YOZ}$ color space. After preprocessing and color transformation, the videos are decomposed using $8 \times 8$ DCT blocks, making the spatial decomposition of the sequence equivalent to 64 different frequency and orientation channel decomposition. The local contrast of the decomposition blocks is adjusted based on their DC values. A single temporal channel is obtained after the spatial decomposition using a single $2^{\text {nd }}$-order IIR filter, designed to minimize the time and memory requirements of the algorithm. A JND sequence is generated by dividing the 
decomposed video channels by their maximum threshold then a difference sequence is obtained.

Watson used only intra-channel masking in his algorithm. To apply masking to the difference sequence, a mask is created from decomposing the reference sequence and filtering it temporally using a $1^{\text {st }}$-order IIR filter with gain $g$, and time delay $\tau$ (Watson used $\tau=0.04$ second to fit data from Georgeson and Georgeson [58]). The result is then raised to the power of $m$ and any values less than 1 is replaced with 1 , mimicking traditional masking where values less than the threshold are not affected and those above it are raised to the $m^{\text {th }}$ power [33]. Similar to other algorithms, the final quality metric is obtained through Minkowski's pooling of the masked difference errors.

\subsubsection{Winkler's perception difference metric}

The perception difference metric (PDM) was proposed by Stefan Winkler in 1999 [32] as an algorithm for colored video quality prediction. The algorithm, shown in Figure 2.11, starts by transforming the color space of the video sequence to the blackwhite (B-W), red-green (R-G) and blue-yellow (B-Y) color space. This opponentcolors space was chosen by Winkler since pairs of color hues can coexist in a single color sensation, as first indicated by [59] and later supported by psychological and physiological experiments such as $[60,61]$.

After the color space pre-processing step, the sequence is tempo-spatially decomposed. The decomposition is achieved by first decomposing the sequence into two temporal channels (a sustained low frequency channel and a higher frequency transient channel) followed by a five level four orientation spatial decomposition. The temporal decomposition was computed through the use of IIR filters designed to minimize time delay. The lowpass sustained channel filter is used to decompose all 


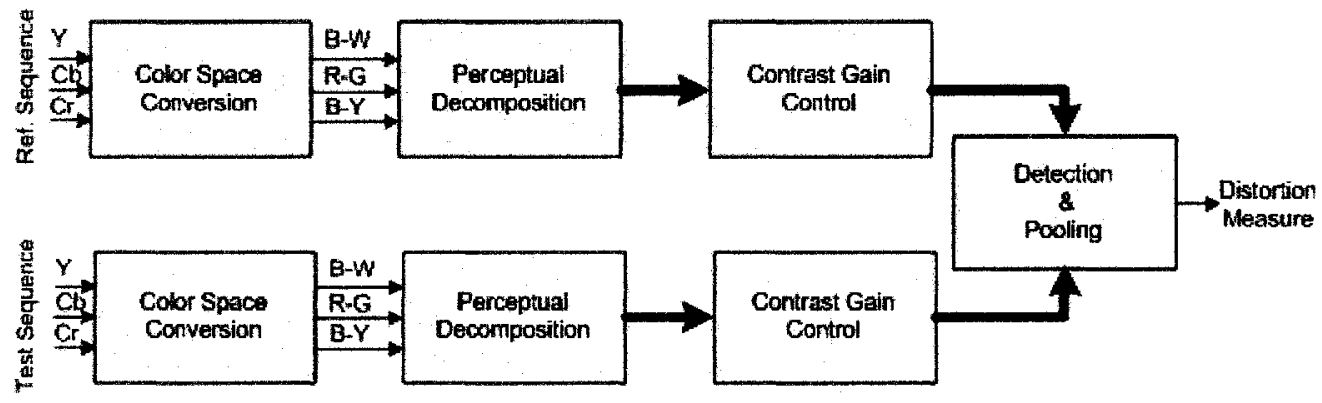

Figure 2.11: Block diagram representation of Winkler's perception difference metric $[32]$.

color information. However, the transient channel filter is only applied to the B-W color information, to save computation time since the HVS is less sensitive to color in high frequencies. The spatial decomposition is achieved using Simoncelli et al.'s steerable pyramids [62] and is applied to all the temporal decomposition channels from all colors.

The decomposed sequences are weighted to approximate the contrast sensitivity of the HVS. An error signal is generated after applying masking to all the orientation channels of each decomposed frame. To get the final distortion metric, two pooling steps are used. An $L_{2}$-norm is calculated for the pixels of all channels, to get the distortion metric of each individual frame. Once the frame distortions are calculated, an $L_{4}$-norm is used to get the video's distortion metric from the different frames' distortions.

Winkler's PDM, Sarnoff's JND (Sec. 2.7.3) and Watson's DVQ metric (Sec. 2.7.4) were tested and reported in the VQEG phase I report [63] to have very good correlation with subjective tests results. For a thorough comparison between the three algorithms, see [13]. 


\subsubsection{NTIA general video quality assessment model}

The general video model (GVM) was developed in 2000-2001 by the National Telecommunication and Information Adminstration (NTIA) and International Telecommunication Union (ITU) as part of their video quality assessment models. Three other models were developed along with the GVM; television model, video conferencing model and a developer's model. The GVM was targeted to be a general video quality assessment algorithm, covering a wide range of video impairments. Although NTIA/ITU's GVM is considered to be a reduced reference (RR) model, it was proponent $\mathrm{H}$ in the VQEG's phase-II full reference television test [41]. The GVM was developed as a RR model to allow for real-time quality assessment of transmitted video sequences, given that a separate transmission channel for the extracted information can be provided.

The algorithm, which operates in the YCbCr color space, starts by a calibration process for the test sequence before extracting any quality assessment parameters. The calibrating stage takes care of spatial alignment, temporal alignment, gain and level offset compensation and valid video region (VVR) estimation. The VVR refers to the area of the video that is displayed and perceived by viewers. It is the region that contains valid picture information. Defining the VVR is important, as the test video should not be penalized for information corruption or distortions in areas that will not be displayed or perceived by viewers, hence, not affecting the viewer's opinion of the video sequence. In the GVM, the spatial alignment must be taken care of before any of the other calibration variables. However, the four mentioned calibration variables are interdependent. This means that if we are to accurately estimate the gain offset, for example, we have to have correctly corrected for the spatial and temporal shifts as well as correctly identified the sequences VVR. Since performing a full exhaustive search over all the variables is inefficient, slow and computationally expensive, the 
GVM uses an iterative search technique to find the best matched frame spatially. The iterative search is composed of a broad search step, a narrow search step and fine-tuning step allowing for accuracy of up to 0.5 pixel shifts. Once spatial shifts are compensated for, the other calibrating variables are accounted for as discussed in [15]. It is important to note here that although spatio-temporal shifts and luminance gain are corrected, gains in the chromatic color information are not corrected as they are considered impairments for which the sequence should be penalized.

At its core, and after the videos are calibrated, the GVM video quality metric (VQM) is obtained by combining different parameters generated from the video sequences. The parameters used are obtained through a process that can be generalized in the following five steps: (1) a perceptual filter is used to enhance certain perceptual features, then the sequence is divided into small spatio-temporal blocks, (2) features are generated and extracted from the perceptually enhanced sequence via simple mathematical functions (normally the mean or the standard deviation function is used), (3) perceptual thresholding is applied, (4) reference and test sequence information are compared, and (5) a spatial and temporal collapsing step is used to combine the individual spatio-temporal blocks' parameters into a single parameter. For the comparison step, one of the following three comparison functions is used, the Euclidian distance (2.9), ratio comparison (2.10) or the log comparison function (2.11) is used.

$$
\begin{gathered}
p=\sqrt{\left(f_{r}+f_{t}\right)^{2}+\left(f_{r 2}+f_{t 2}\right)^{2}} \\
p=\left(f_{t}+f_{r}\right) / f_{r}
\end{gathered}
$$




$$
p=\log _{10}\left(\frac{f_{t}}{f_{r}}\right)
$$

where $f_{r}$ and $f_{r 2}$ are reference sequence's features information, and $f_{t}$ and $f_{t 2}$ are corresponding test sequence feature information.

The GVM uses seven parameters that were calibrated with subjective scores and weighted to produce the best quality prediction. The VQM, which has a lowest quality score of 0 and highest quality score of 1 , is calculated as

$$
\begin{aligned}
V Q M= & -0.2097 P 1+0.5969 P_{2}+0.2483 P_{3}+0.0192 P_{4} \\
& -2.3416 P_{5}+0.0431 P_{6}+0.0076 P_{7}
\end{aligned}
$$

where the parameters

- P1 accounts for spatial information loss, such as blurring, through measuring the changes in the perceptually significant edge information.

- P2 detects information shift from horizontal and vertical edges to the diagonal orientation, detecting if the horizontal or vertical edges were blurred more than the diagonal edges. This is important since the HVS is less sensitive to diagonal artefacts than it is to vertical or horizontal ones.

- P3 working in the opposite direction of $P_{2}$, detects information shift from diagonal orientation to the vertical and horizontal edges, allowing for detecting blocking artefacts.

- P4, which is generated by measuring the Euclidian distance between the chromatic features of the reference and test sequences, detects changes in the spread 
and distribution of the 2D color information between the test and reference sequences.

- P5, differing from other parameters in that it is used to increase the quality rating. This parameter rewards for enhancements made to the test sequence's perceptual features. Some constraints are added to the parameter to prevent it from giving the test sequence a higher quality than its reference.

- P6 is used to adjust the quality based on the spatial and temporal details. The parameter was included as the HVS sensitivity to impairments changes based on the amount of spatial features or amount of motion in the scene. To calculate $P 6$, a product of two streams, one for motion information and one for contrast information, is generated for each of the two video sequences. $P 6$ is taken as the ratio between the reference and test sequence's streams.

- P7 is used to account for localized color impairments that might occur due to transmission errors.

The algorithm showed high correlation results in the VQEG's phase-II tests. NTIA's GVM was not tested and compared using a wide set of video impairments. However, as the algorithm was designed and calibrated using a wide range of video impairments, it is expected to perform in a similar manner to that in the VQEG phase-II testing for any video impairment. For more information about the calibration process, the parameters' description and the features extraction and parameter generation steps, we refer the reader to the algorithm's detailed discussion in [64]. A summaries discussion of the algorithm and its results can also be found in [15]. 


\subsubsection{Wavelet-based video visible distortion measure}

Proposed by Yao et al. in [65] as a wavelet-based video visible distortion measure (WV-VDM). The algorithm uses the Cohen-Daubechies-Feauveau 9/7 (CDF 9/7) bi-orthogonal wavelet transform in decomposing the video sequences. Also, similar to $[10,57]$, the algorithm uses the just noticeable difference profile in assessing the quality of the given video sequence. The WV-VDM, at its core, is the sum of the sequence's visible error adjusted based on different masking functions.

The HVS is less sensitive to artefacts in high frequency bands (fast motion), diagonal orientation bands (close to $45^{\circ}$ ), extreme luminance values (very bright or very dark) or regions with very high content/activity. For that, three masking functions were used to enhance the quality algorithm; contrast masking, luminance masking and temporal masking. The contrast masking function, $C(x, y)$, used was Winkler's isotropic local contrast function proposed in [35]. Luminance masking, $L(x, y)$, for each decomposition level was calculated through a power function, using the coefficients of the $L L$ band of that level and the band's mean luminance value. To account for interframe differences/motion (higher motion means higher masking effect), a temporal masking function, $T(x, y)$, is calculated from interframe luminance differences of consecutive frames.

Once the JND profile and the masking functions have been defined, the perceived visual quality (PVQ) of each frame is calculated over all the frame decomposition levels $(\lambda)$, orientations $(\theta)$ and coefficient locations $(x, y)$ using

$$
P V Q=\sum_{\lambda, \theta, x, y} \frac{\left[\left(\left|w_{\lambda, \theta}(x, y)-\hat{w}_{\lambda, \theta}(x, y)\right|-J N D_{\lambda, \theta}\right)^{2} \cdot \delta(x, y)\right]^{0.5}}{L_{\lambda}(x, y) C_{\lambda}(x, y) T_{\lambda}(x, y)}
$$

where $w_{\lambda, \theta}$ and $\hat{w}_{\lambda, \theta}$ denote the wavelet coefficients of the reference and test video, 
respectively, and

$$
\delta(x, y)= \begin{cases}1, & \text { if }\left|w_{\lambda, \theta}(x, y)-\hat{w}_{\lambda, \theta}(x, y)\right|>J N D_{\lambda, \theta} \\ 0, & \text { otherwise }\end{cases}
$$

The overall visual quality is calculated over all of the sequence's frame $P V Q$ values through Minkowski pooling. The performance of the WV-VDM algorithm was evaluated using the test video sequences of the VQEG's phase-I test [63]. The proposed algorithm, as reported in [65], showed better performance than all the proponents used in VQEG's phase-I test.

The approach used in WV-VDM is similar to that used in the wavelet-based image quality algorithms proposed in [11] and [12], both discussed briefly in Sec. 2.6.8 and Sec. 2.6.9, respectively. Although the WV-VDM algorithm was developed for and tested using digital video sequences, it cannot be considered a true video quality assessment algorithm. This is because the algorithm uses a 2D spatial wavelet decomposition with no temporal decomposition and both contrast and luminance masking are derived from information based on each individually evaluated frame. Also, the only temporal element in the algorithm is the temporal masking which, based on temporal information between consecutive frames, is used to reduce the contribution of each frame's spatial errors to the final frame's quality. Another very important issue making the WV-VDM more of an image than video quality assessment algorithm is the lack of a temporal distortion evaluation mechanism needed to account for errors between the temporal changes of the reference and test sequences. We have included this algorithm in our discussion for two reasons: the algorithm's reported good quality prediction and the algorithm's simplicity of design. The simplicity of design allows for algorithm integration with little overhead to most wavelet compression algorithms. 
We believe that the algorithm can be further enhanced through the incorporation of temporal decomposition and/or a temporal artefact evaluation process.

\subsubsection{Motion models based video structural similarity metric}

Inspired by the structural similarity (SSIM) algorithm proposed by Wang et al. in $[3,17]$ and its complex wavelet extension (CW-SSIM) $[20]$ (both are detailed in the next chapter), Seshadrinathan and Bovik in [2] proposed a FR extension to the image SSIM index, dubbed V-SSIM, that moved the algorithm to the spatio-temporal complex wavelet domain and utilized motion models, to assess digital videos quality. Motion models were used in the V-SSIM algorithm, as it is believed that introducing meaningful motion information can enhance the performance of the video quality assessment algorithm.

Complex motion can be represented as simple translations when considering small sections of the video sequence. Also, as discussed in [2], when working in the frequency domain, simple motion can be seen as shearing to the magnitude of the spatial frequencies and not as a change in their values. This, along with the frequency based perceptual channel decomposing of the HVS and the success of the CW-SSIM algorithm, made working in the frequency domain, for calculating the motion models and predicting the video quality, an attractive choice. The motion models selected to be incorporated in the V-SSIM algorithm were the optical flow information, as they can be calculated using phase information in the frequency domain.

The V-SSIM algorithm uses a family of 22 Gabor filters to perform the channel decomposition. The Gabor filters were designed to have the same geometry as the ones used by Fleet and Jepson [66]. With this filter bank selection, the same filter family can be used to decompose the video sequence into different channels/frequency planes, and to calculate the optical flow information for the sequence. The algorithm works by 
first filtering both the reference and test video sequences using the Gabor filter family, resulting in 22 different frequency coefficient planes (one for each filter). Optical flow information, which estimates the orientation of the plane containing the frequency spectrum of that part of video sequence, is calculated. Optical flow information is used to select the Gabor filter that is the closest, within 1 standard deviation, to the motion plane. Once the filter is identified, the coefficients are extracted from it to be used in the quality assessment algorithm. The extracted coefficient set can be considered as a motion compensated filtered video sequence.

V-SSIM uses a function similar to that proposed in [2]. However, the V-SSIM function defers in that it does not include the phase information. The phase information represents translations in the image information. However, in videos, translations are motion. Since motion is compensated for with the optical flow calculation, phase information is not included in the final quality assessment function. Let $\vec{R}=r_{k}, i=1,2, \ldots, N$ denote the set of coefficients from the Gabor filter family. Similarly, let $\vec{T}=t_{k}, i=1,2, . ., N$ be the corresponding coefficients from the test video sequence. Now, let $C$ be the set of filter indices of selected filters using the optical flow information. Then the quality index of the video sequence is given by

$$
\operatorname{V-SSIM}(\vec{R}, \vec{T})=\frac{2 \sum_{k \in C}\left|r_{k}\right|\left|t_{k}\right|+K}{\sum_{k \in C}\left|r_{k}\right|^{2}+\sum_{k \in C}\left|t_{k}\right|^{2}+K}
$$

where $K$ is a small stabilizing constant.

Fleet and Jepson's algorithm [66], which is used to calculate the optical flow information used in the V-SSIM algorithm, does not yield $100 \%$ optical flow density. To overcome this problem, spatial quality was calculated for regions with no optical flow information and areas with no motion. The algorithm is limited due to the filter design and is unable to predict the quality of video sequences with fast motion. 
Nonetheless, the algorithm showed good correlation with subjective scores, when evaluated with a subset of the video sequences used by VQEG tests (the sequences with fast motion were not used in the algorithm's evaluation). The V-SSIM algorithm depends on the accuracy of the optical flow estimation, as the wrong optical flow information will result in the use of the wrong subset of video coefficients. Also, the denser the generated optical flow information obtained for the sequence, the better the prediction of the algorithm is expected to be. A multi-scaled version of the algorithm might be under development, as mentioned in [2], to allow the algorithm to work with sequences containing fast motion. 


\section{Chapter 3}

\section{The Structural Similarity Index}

Since the final receiver of processed images and videos are human viewers, it comes natural that the HVS is considered in the design of image or video quality assessment algorithms. HVS based algorithms are built to incorporate the known characteristics of the HVS such as the perceptual channel decomposition, contrast sensitivity and distortion masking (see Chapter 2, Sec. 2.1 and Sec. 2.5). However, aside form the fact that known HVS characteristics still need more study, including such characteristics in a quality assessment algorithm generally result in a slow and very complicated system.

Along side the complexity and time requirement of perceptual based quality assessment algorithms, they suffer from a number of limitations. Some of the limitation of perceptual algorithms, as outlined in [3], are:

- The Quality Definition Problem: A fundamental problem to any quality assessment algorithm. This problem is about defining the quality relation with the visible errors in the image/video. In particular, the problem is in defining the amount of effect a certain visible error is to have on the final quality. Not all visible errors should be treated the same way, as some errors may be annoying (blocking) and other may not affect the viewer's opinion of the viewed scene 
(shift in luminance). To our knowledge, there has not been any experiments that tested and quantified the effect of each catcgory of visible errors on the general opinion score.

- The Suprathreshold Problem: Error sensitivity measures, such as the CSF and masking, are derived from psychophysical experiments designed to calculate the barely noticeable error thresholds. However, such experiments do not study the effects that distortions significantly above the near-visible threshold might have nor do they analyze suprathreshold effects between different perceptual channels.

- The Natural Image Complexity Problem: This is more of a concern than a problem. Psychophysical experiments use simple patterns, spots and bars, when testing HVS characteristics which are used to derive visual models (CSF normally is derived using simple sinusoidal functions and masking is derived by superimposing a few simple patterns). A concern can be raised in the sufficiency of these simple/limited tests in deriving models that represent more complex natural images. Efforts are being made to build a database with images containing both simple and more complex patterns which will facilitate studies that might shed more light on this issue [67].

- The Decorrelation Problem: This limitation is a result of the choice of the pooling method used in perceptual algorithms. Almost all assessment algorithms use Minkowski's summation in combining the different errors into a single error value. The use of Minkowski's summation assumes that errors are independent from each other. It is known that errors in the different perceptual channel are not independent and there are relationships between intra- and inter-channel coefficients, especially when a linear channel decomposition is used such as the 
wavelet transform. To overcome this limitation, a different pooling method, that accounts for the dependencies of errors across channels, must be defined and used. Other methods that can be used to reduce the effect of this limitation is: the use of a well defined inter-channel masking, and the use of non-linear gain control models to reduce the dependencies between different channel's coefficients/errors.

- Cognitive interaction Problem: This problem relates to the effect the cognitive processing of the scene has on its perceived quality. The quality of some image or video can be different depending on the viewer's expectation, the prior knowledge of the information in the scene, the exposure time (some impairment might become less noticeable with time while others might become more noticeable). This behavior of cognitive processing is not included in quality models due to the lack of its definition and the difficulty in quantifying it. We believe that a cognitive study, designed to assess different impairment's effects on perceived quality under different viewer preparation and exposure time, might result in the design of better performing quality systems.

To address the limitation associated with current quality assessment approaches and with the need for simpler yet effective quality assessment algorithms, a different approach of looking at the HVS emerged. Recognizing that the HVS is more adapted to extract structure information from the viewed scene than detecting absolute differences in intensities, Wang et al. proposed the universal image quality index [17] which was later developed to become the structural similarity (SSIM) index [3]. The SSIM index showed to have very good image quality prediction and led to the development of a family of assessment algorithms [2,18-20,68].

In this chapter, we will discuss the SSIM index and its extension to the complex 
wavelet transform $(\mathbb{C W T})$ domain. Also, the chapter will discuss the use of the SSIM index in objective video quality assessment.

\subsection{SSIM Index: An Image Quality Assessment Algorithm}

By changing the direction quality algorithms are looked at from a bottom-up to a top-down direction, a new ideology of developing algorithms is developed. Using the top-down approach, the overall HVS is looked at, instead of trying to quantify the early steps used by the HVS in processing a perceived scene. When considering the overall perceptual system, it can be seen that it is more adapted towards extracting the structural information from the viewed scene rather than trying to quantify each stimulus or evaluating individual intensity differences/errors .

Certain perceived quality behavior can be better explained using the structure information of a scene instead of using visible error differences. The negative effect on the perceived quality of a scene due to artifacts, such as blurring or added noise, can be explained using an absolute error approach, as a differenc/error in the scene, as well as using a structural based approach, as a change in the scene's structure. However, a luminance shift artifact, for example, not affecting the perceived quality of the overall scene can be better explained through a structure approach rather that an error based one. Figure 3.1 shows quality results from an error based approach, using MSE, and the structure based SSIM index.

Utilizing the structure information in a top-down approach when assessing a scene's quality can reduce or eliminate some of the quality assessment problems and 


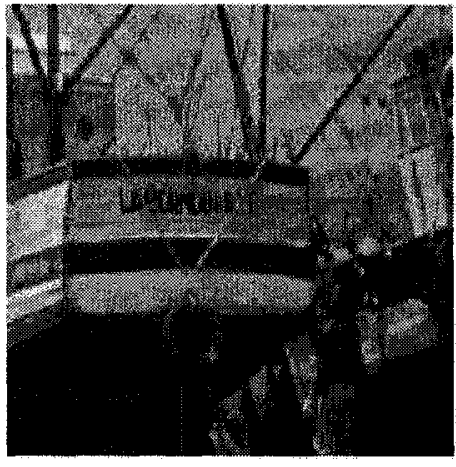

(a)

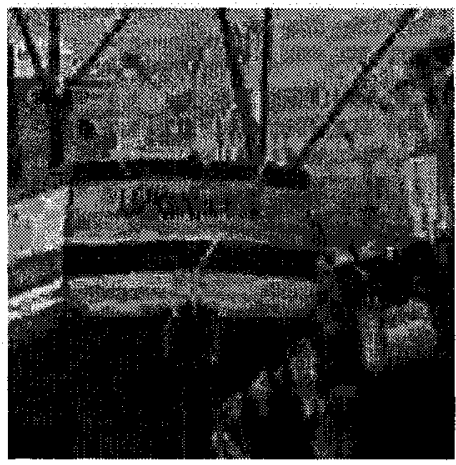

(d)

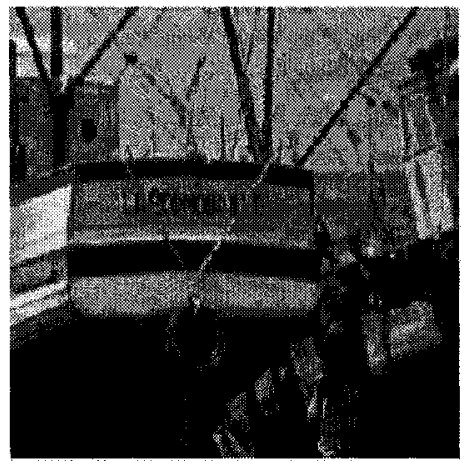

(b)

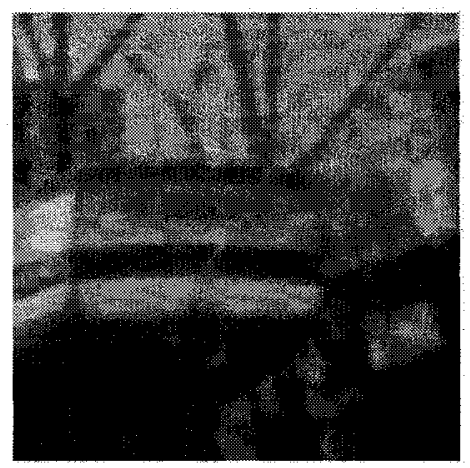

(e)

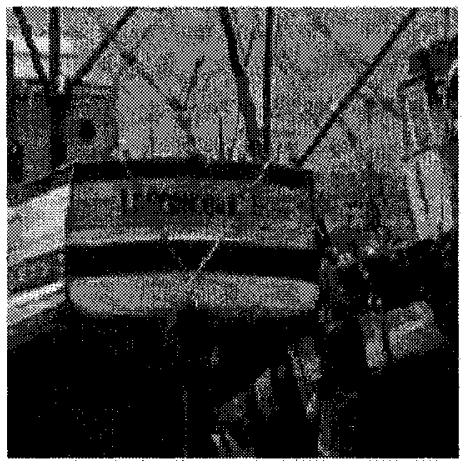

(c)

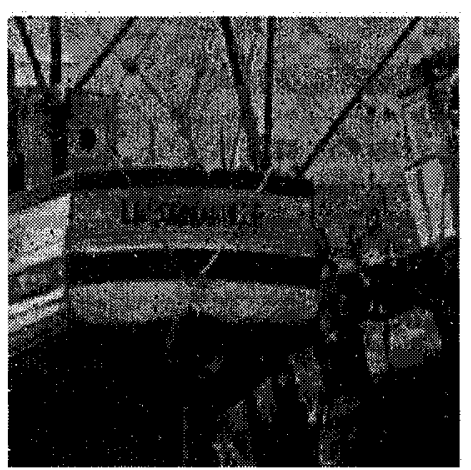

(f)

Figure 3.1: Comparison of Boat images with different types of distortions, all with $\mathrm{MSE}=210$. (a) Original image ( $8 \mathrm{bits} /$ pixel; cropped from $512 \times 512$ for visibility). (b) Contrast-stretched image, mean SSIM (MSSIM) $=0.9168$. (c) Mean-shifted image, MSSIM $=0.9900$. (d) JPEG compressed image, MSSIM $=0.6949$. (e) Blurred image, MSSIM $=0.7052$. (f) Salt-and-pepper impulsive noise contaminated image, MSSIM $=0.7748$. (all from [3]). 


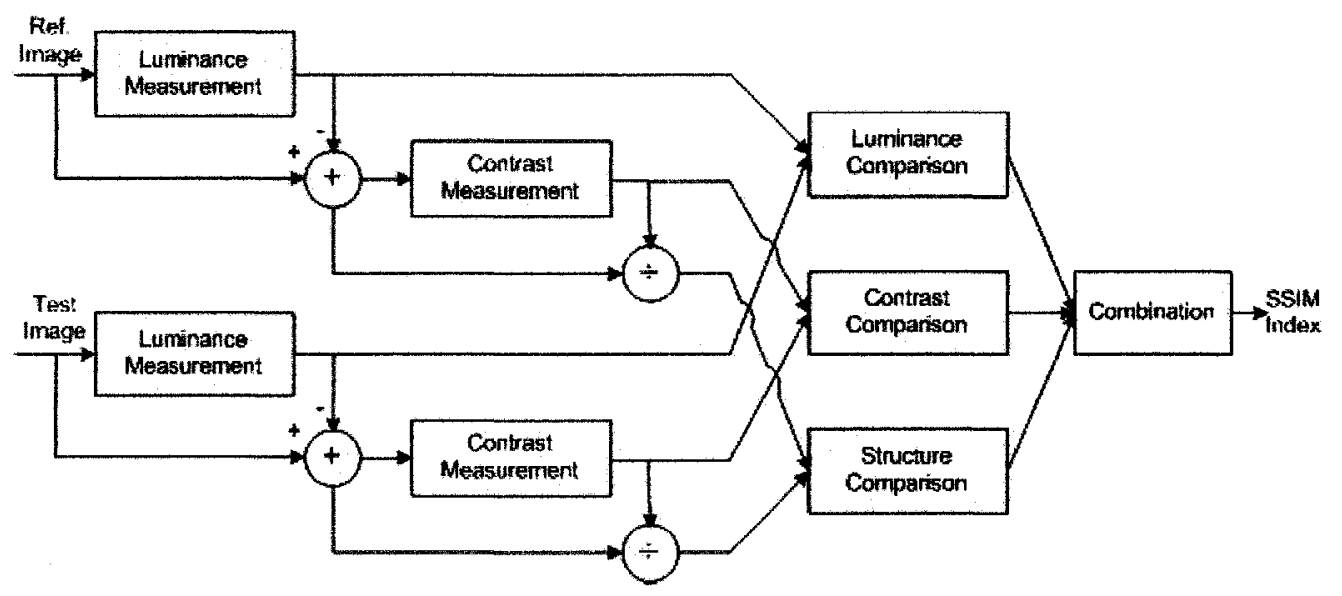

Figure 3.2: Diagram of the structural similarity (SSIM) measurement system [3]

limitations. Since the scene is not evaluated based on absolute perceived errors, problems such as the suprathreshold is eliminated. Also, the cognitive problem can be reduced when evaluating the quality of the scene from its complex structure instead of evaluating it from errors obtained using the understanding of the HVS response to simple patterns.

Exploiting the fact that the HVS is more adapted towards extracting structure information from the viewed scene, Wang et al. developed the structural similarity (SSIM) index [3]. The SSIM algorithm estimates the quality of an image based on the differences between its structure information and that of its reference image (considered to be of perfect quality).

The structure information is defined as the attributes of the scene that are independent from the scene's luminance and contrast. This means that to be able to compare the structure information in a given scene, the luminance and contrast values have to be removed and the structure information extracted. Since the luminance and contrast values vary through out the scene, local luminance and the contrast are used in the SSIM algorithm. 
The SSIM algorithm, as illustrated in Figure 3.2, calculates the local luminance first as the local mean of the scene.

$$
\mu_{x}=\frac{1}{N} \sum_{i=1}^{N} x_{i}
$$

Second, the luminance is removed from the scene and the local contrast is estimated using the standard deviation function.

$$
\sigma_{x}=\left(\frac{1}{N-1} \sum_{i=1}^{N}\left(x_{i}-\mu_{x}\right)^{2}\right)^{0.5}
$$

Finally, the luminance shifted scene, $x-\mu_{x}$, is normalized using $\sigma_{x}$ and the remaining attributes are taken as the structure information. With that, the structure information of a scene is defined as

$$
\varsigma_{x}=\left(x-\mu_{x}\right) / \sigma_{x}
$$

For two images $x$ and $y$, where one is considered to be the perfect quality image and the other is the distorted version of the same image, the SSIM quality index is calculated using three comparisons: luminance, contrast, and structure. All three components were considered in calculating the SSIM quality index as they are relatively independent (for example changing the luminance will not change the contrast or the structure information),

The SSIM index, $\operatorname{SSIM}(x, y)$, for a local scene is taken as a function of the luminance comparison function $l(x, y)$, a contrast comparison function $c(x, y)$ and structure comparison function $s(x, y)$. The function is defined to meet three constraints: (1) symmetry: $\operatorname{SSIM}(x, y)=\operatorname{SSIM}(y, x)$, (2) boundedness: $\operatorname{SSIM}(x, y)<1$ and (3) unique maximum: $\operatorname{SSIM}(x, y)=1$ if and only if $x_{i}=y_{i}$ for all $i=1,2, \ldots, N$, $N$ is the number of pixels in the scene. 
The luminance comparison function, $l(x, y)$, was defined as

$$
l(x, y)=\frac{2 \mu_{x} \mu_{y}+C_{1}}{\mu_{x}^{2}+\mu_{y}^{2}+C_{1}}
$$

and similarly the contrast comparison function, $c(x, y)$, was defined as

$$
c(x, y)=\frac{2 \sigma_{x} \sigma_{y}+C_{2}}{\sigma_{x}^{2}+\sigma_{y}^{2}+C_{2}}
$$

where $C_{1}$ and $C_{2}$ are small constants used for stability.

The definition used for $l(x, y)$ and $c(x, y)$ meets the three requirements. It can be seen that this definition makes $l(x, y)$ consistent with Weber's law that is widely used for light adaptation. Also, the definition makes $c(x, y)$ less sensitive to the same amount of contrast change $\Delta \sigma=\sigma_{y}-\sigma_{x}$ in high base contrast $\sigma_{x}$ than low base contrast. This feature is very attractive as it simulates the masking behavior of the HVS.

The structure comparison function is taken as the correlation (inner product) between the structure information, given by (3.3) of the reference and test scene's structure attributes. The correlation is used as it is an effective yet simple measure of the structure similarity. The structure comparison function is defined as

$$
s(x, y)=\frac{\sigma_{x y}+C_{3}}{\sigma_{x} \sigma_{y}+C_{3}}
$$

where $C_{3}$ is a small positive constant used for algorithm stability and

$$
\sigma_{x y}=\frac{1}{N-1} \sum_{i=1}^{N}\left(x_{i}-\mu_{x}\right)\left(y_{i}-\mu_{y}\right)
$$

The three functions are combined to calculate the local SSIM index as 


$$
\operatorname{SSIM}(x, y)=l(x, y)^{\alpha} \cdot c(x, y)^{\beta} \cdot s(x, y)^{\gamma}
$$

where $\alpha, \beta$ and $\gamma$ are positive constant weights. The SSIM quality index has a range of 0 to 1 with the maximum value of 1 achieved only when $x$ and $y$ are identical. Setting $\alpha, \beta$, and $\gamma$ to 1 for simplicity and substituting $l(x, y), c(x, y)$, and $s(x, y)$ in (3.8) with their definitions from (3.4), (3.5) and (3.6), respectively, we get:

$$
\operatorname{SSIM}(x, y)=\frac{\left(2 \mu_{x} \mu_{y}+C_{1}\right)\left(2 \sigma_{x y}+C_{2}\right)}{\left(\mu_{x}^{2}+\mu_{y}^{2}+C_{1}\right)\left(\sigma_{x}^{2}+\sigma_{y}^{2}+C_{1}\right)}
$$

The SSIM index is applied and calculated for local image windows. Although large windows allow for accurate statistical analysis of the image, they make the algorithm less sensitive to fine distortions. On the other hand, small window sizes will make the algorithm inaccurate in calculating the distortions and predicting the quality. The SSIM algorithm is applied to images locally using $8 \times 8$ overlapping sliding window fashion. The SSIM index, local-quality, is calculated for each small window using (3.9). The overall SSIM quality index for the entire image is calculated as the mean SSIM (MSSIM) of all the local values using

$$
\operatorname{MSSIM}(X, Y)=\frac{1}{M} \sum_{i=1}^{M} \operatorname{SSIM}\left(x_{i}, y_{i}\right)
$$

where $X$ and $Y$ are the reference and distorted images respectively, $x_{i}$ and $y_{i}$ are the images blocks at the $i^{\text {th }}$ window, and $M$ is the number of local windows in each image.

The SSIM algorithm gave higher correlation values using person's linear correlation and Spearman's rank-order correlation test than PSNR [3,69,70]. Also, the dense intermediate local-index quality values, generated from the overlapping windows, are used to build a quality map of the distorted image. The SSIM map highlights the 


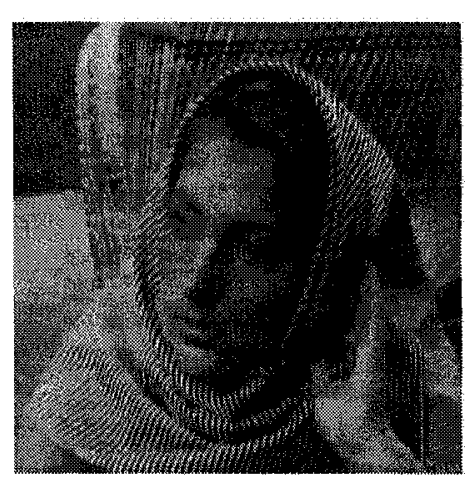

(a)

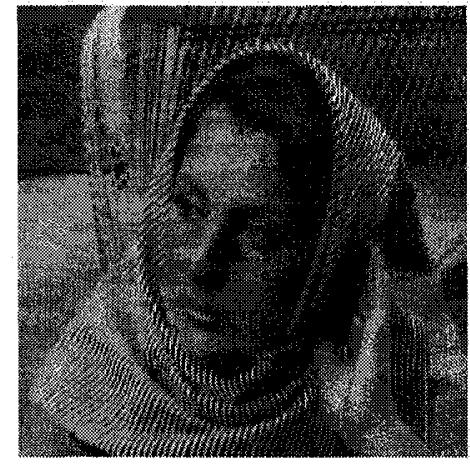

(b)

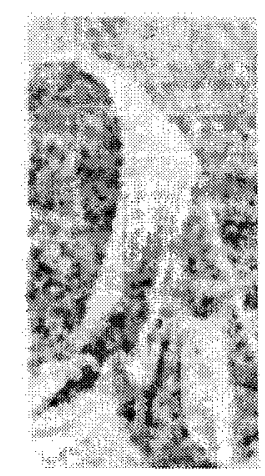

(c)

Figure 3.3: Comparison of Barbara images showing the resulting SSIM quality map. (a) Original image ( $8 \mathrm{bits} /$ pixel, original size of $256 \times 256$ ). (b) $30 \mathrm{dBW}$ white Gaussian noise added only to the right half of the image, MSSIM $=0.8432$. (c) SSIM map of the noisy image. Brightness indicates the magnitude of the local SSIM index, with bright indicating high quality and dark indicating poor quality.

high and low quality regions using bright and dark luminance values, respectively. Figure 3.3 shows an example of SSIM map generated from the "Barbara" image with white Gaussian noise of power $30 \mathrm{dBW}$ added only to the right half of the original image.

\subsection{Moving SSIM to the Complex Wavelet Do- main}

The SSIM algorithm, although capable of detecting a wide rang of distortions, has shown that it is not very sensitive to changes in the image's luminance or contrast, as such distortions are not structural and do not affect the perceptual quality of the image (see images (b)-(g) in Figure 3.4). Geometrical distortions (translation, rotation and scaling) are not structural distortions and do not affect the perceived quality when present in the image in small amounts. Nonetheless, quality assessment 
algorithms, including the structural based SSIM index, are highly sensitive to such geometric distortions as shown with images (h)-(l) in Figure 3.4.

Since geometric distortions should not affect the final quality predicted by the assessment algorithm, one of two approaches can be used [20]: (1) registration approach which is normally performs as a pre-processing step, before the actual quality assessment algorithm is applied, to estimate the amount of translation, rotation and/or scaling in the signal and apply its inverse. (2) using an invariance approach. An invariance approach is one where the quality parameter is derived using a set of measurements that are invariant to such geometric distortions.

To enhance the performance of SSIM, non-structural geometric distortion have to be accounted for without affecting the algorithm's low sensitivity to luminance and contrast changes. Including a registration step to the current implementation of the SSIM algorithm provides a solution to the high geometrical distortion sensitivity problem. However, accurately defining the translation, rotation or scaling parameters can be computationally intensive and time consuming. The registration complexity is further elevated if the image contains more than one category of geometric distortion and/or contains artifacts that affect the structure of the image, which also would reduces the registration's accuracy. Due to the overhead associated with image registration, moving the SSIM algorithm from its spatial domain which is very sensitive to geometric artifacts, to one that is more robust to such distortions becomes attractive.

Recognizing that any changes in an image can be represented as a magnitude and/or phase change in the complex frequency domain, Wang et al. proposed moving the SSIM algorithm from its spatial domain to the complex wavelet transform (CWT) domain. The $\mathbb{C W T}$ was chosen since it retains the feature localization of the wavelet transform, allowing for a multi-scale analysis similar to that in the spatial domain, 


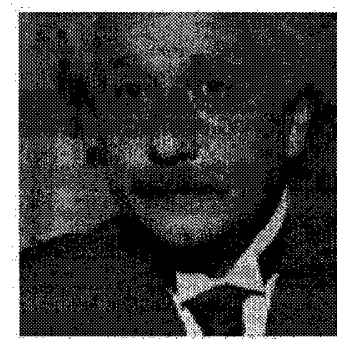

(a) MSE $=0, \mathrm{SSIM}=1$ CW SSIM=:

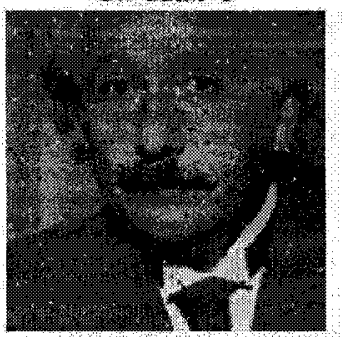

(6) MSE $=313, \mathrm{SSiM}=0730$ CW-SSIN=0811

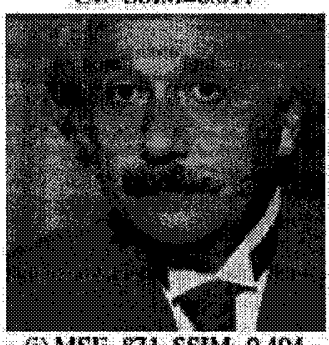

(1) MSE $=871,5 \mathrm{sIM}=0.404$ cw ssiM $=0.933$

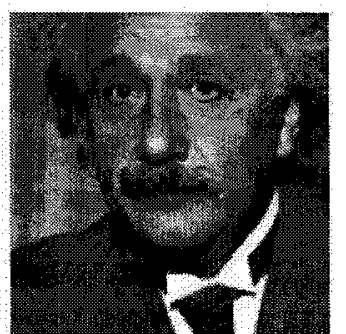

(b) MSE $-306,5614=0928$ CV ssm $=0.988$

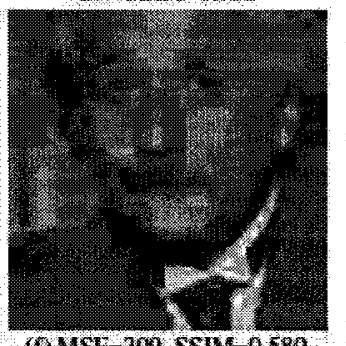

WMSE $=36$, SSIM $=0.580$ CW SSM $=0.633$

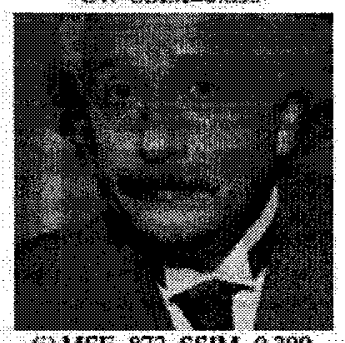

6) MSE $=873.551 \mathrm{M}=0.399$ CW SSIM $=0.933$.

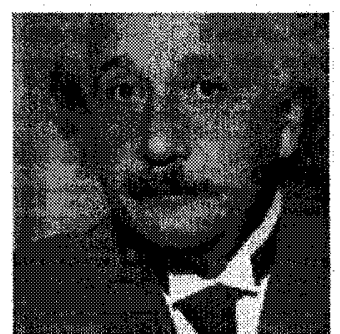

(c) MSE $-309.5 \mathrm{SIM}=0.987$ CW SSIM $=1.000$

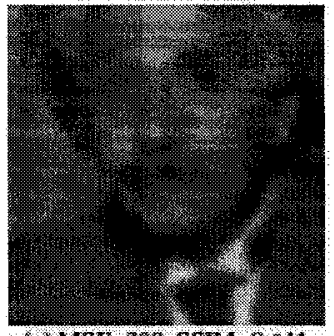

(1) MSE-308, $\operatorname{sSTM}=0.64$ CW-ssiM=06a

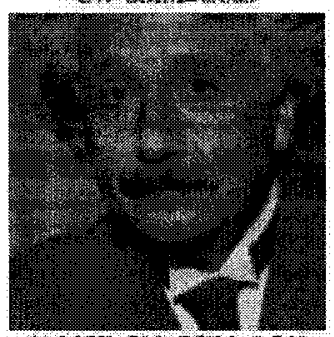

(X)MSE=590, SSMM $=0549$ CW-SSIM-0917

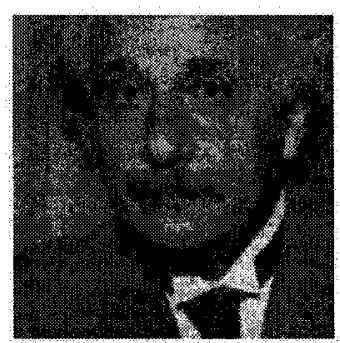

(d) MSE $=309, \operatorname{sSM}=0576$ CW-SSIM $=0.814$

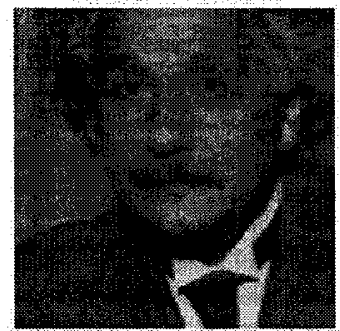

(h) MSI $=694.5 \mathrm{sM}=0505$ CW $\mathrm{SSIM}=0.925$

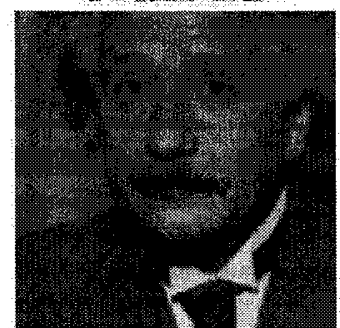

(D) MSE $=577$, SIIM $=055$ CW $\$$ sIM-0.916

Figure 3.4: Comparison of image similarity measures for images with different types of distortions (Images were cropped from $256 \times 256$ to $128 \times 128$ for visibility). (a) reference image ( 8 bits/pixel, assumed to have perfect quality); (b) contrast stretch; (c) mean luminance shift; (d) Gaussian noise contamination; (e) impulsive noise contamination; (f) JPEG compression; (g) blurring; (h) spatial scaling (zooming out); (i) spatial translation (to the right); (j) spatial translation (to the left); (k) rotation (counterclockwise); (l) rotation (clockwise). Note that images (b)-(g) have approximately the same MSE values but drastically different visual quality, which is better predicted by both SSIM and CW-SSIM. Also note that MSE and SSIM are both sensitive to geometrical distortions (images (h)-(l)), which $\mathbb{C W - S S I M ~ i s ~ r o b u s t ~ t o . ~ ( t a k e n ~ f r o m ~ [ 2 0 ] ) ~}$ 
and adds to it a level translation insensitivity to geometric distortions that do not affect the structure of the image.

In the complex wavelet domain, let $\mathbf{c}_{x}=\left\{c_{x, i} \mid i=1,2, \ldots, N\right\}$ and $\mathbf{c}_{y}=\left\{c_{y, i} \mid i=\right.$ $1,2, \ldots, N\}$ be a set of complex wavelet coefficients obtained from the same spatial locations in corresponding complex wavelet decomposition bands of images $x$ and $y$, respectively. The $\mathbb{C W}$-SSIM algorithm is defined as

$$
\mathbb{C} W-\operatorname{SSIM}\left(\mathbf{c}_{x}, \mathbf{c}_{y}\right)=\frac{2 \sum_{i=1}^{N}\left|c_{x, i}\right|\left|c_{y, i}\right|+K}{\sum_{i=1}^{N}\left|c_{x, i}\right|^{2}+\sum_{i=1}^{N}\left|c_{y, i}\right|^{2}+K} \cdot \frac{2\left|\sum_{i=1}^{N} c_{x, i} c_{y, i}^{*}\right|+K}{2 \sum_{i=1}^{N}\left|c_{x, i} c_{y, i}^{*}\right|+K}
$$

where $c^{*}$ denotes the complex conjugate of $c,|\cdot|$ denotes the magnitude operator, and $K$ is a small positive constant for the stability of the algorithm. Similar to the spatial SSIM algorithm, the CW-SSIM index achieves a maximum value of 1 when the two images are identical.

The first part of (3.11), which is used to detect luminance and contrast changes, is completely determined by the magnitude information and has a maximum of 1 if only if $\left|c_{x, i}\right|=\left|c_{y, i}\right|$ for all $i$ 's. Luminance and contrast changes can be roughly modeled as a point-wise linear transform of pixel intensities. When later transformed using a $\mathbb{C W T}$, due to the transform's linear and bandpass nature, can even be represented as $c_{y, i}=$ $a c_{x, i}$, for all $i$ 's. Substituting in (3.11) we obtain $\mathbb{C} W$-SSIM $=(2 a+S) /\left(1+a^{2}+S\right)$, where $S=K / \sum_{i=1}^{N}\left|c_{x, i}\right|^{2}$. From that, keeping in mind that $K$ is a small constant and that $S$ can be ignored for large magnitude values, it can be seen that the algorithm is robust to luminance and contrast changes (a 10\% gain making $a=1.1$ for example, roughly reduces the quality to 0.9955$)$.

The second part of (3.11) is completely determined by the consistency of phase changes and has a maximum of 1 only if the phase differences between $\left|c_{x, i}\right|$ and 
$\left|c_{y, i}\right|$ are constant for all $i$ 's. Since an image's structure is contained in the phase information which is not changed by constant phase shifts, this part of the algorithm is used to measure the structural similarity. Small geometric distortions can be locally estimated by a small translation: $y(u)=x(u)+\Delta u$. This small local translation is represented by a phase shift in the Fourier domain: $Y(\omega)=X(\omega) e^{j \omega \Delta u}$. The same effect is seen in the complex wavelet domain and the local translation is represented by phase shifts in the corresponding wavelet decomposition bands. This means, in the complex wavelet domain, that one of the images is only a phase shifted version of the other.

The CW-SSIMin (3.11) can be simplified by combining the two parts to become

$$
\mathbb{C W S S I M}\left(c_{x}, c_{y}\right)=\frac{2\left|\sum_{i=1}^{N} c_{x, i} c_{y, i}^{*}\right|+K}{\sum_{i=1}^{N}\left|c_{x, i}\right|^{2}+\sum_{i=1}^{N}\left|c_{y, i}\right|^{2}+K}
$$

The CW-SSIM algorithm is shown in related literature, $[5,6,20]$,to have better image qulaity assessment performance that the SSIM algorithm. However, there has not been any work that formally tested and compared the performance of both algorithms using a scored set of varied distortion images. To that, we ran a full quality test using both algorithms. The results are reported in Sec. 5.3.

The CW-SSIM is insensitive to small geometric distortions, as seen in Figure 3.4. However, we must clarify here that the $\mathbb{C W}$-SSIM is only insensitive to small translations, rotations and scaling. This means that if the evaluated image has a moderate to large geometric distortion value (e.g. more the a few pixels of translation or a few degrees of rotation), the algorithm will require a registration step for accurate quality prediction. However, since the algorithm is insensitive to small amounts of geometric distortions, the registration process does not have to be fine-tuned and a rough estimate can be used. 


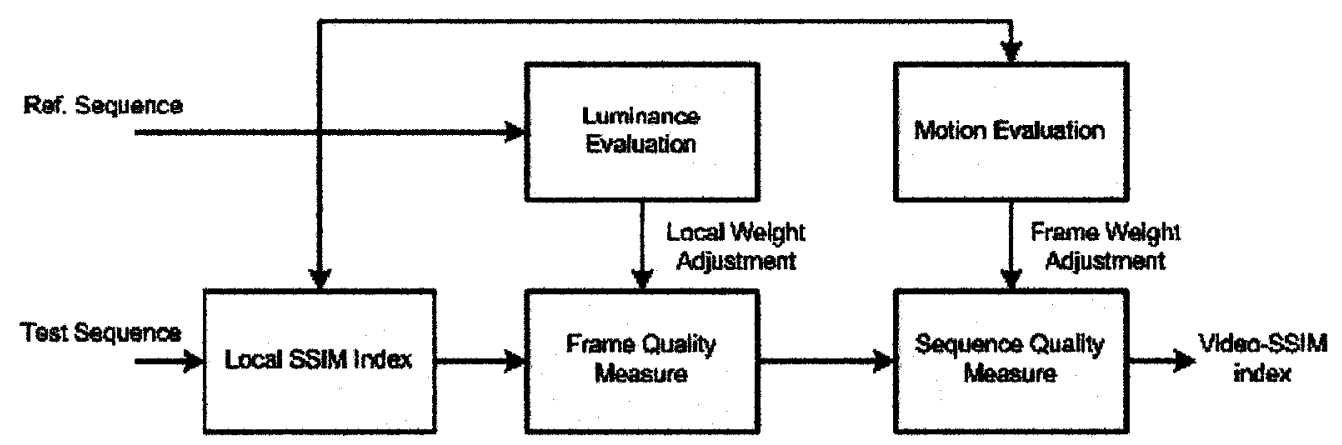

Figure 3.5: Block diagram of the video-SSIM algorithm [19]

\subsection{SSIM in Video Quality Assessment}

The SSIM index, although simple, has shown very good performance in predicting image quality values. The simplicity of the algorithm and its good correlation with subjective scores makes extending the algorithm for video assessment a natural step. To achieve that goal, Wang et al. extended the algorithm to calculate the quality of colored digital video sequences [19].

The video quality index, illustrated in Figure 3.5, is derived as the correlation between the digital video frames in terms of their luminance, contrast, and structure information. However, the Video-SSIM differs from the image SSIM index algorithm by accounting for luminance changes and motion in the video sequence. The HVS attention is focused more towards the brighter regions in the video sequence as well as being more tolerant to artifacts in fast moving objects. To that effect, the video-SSIM uses weights derived from the luminance and motion information in the video frame when combining local SSIM values into an overall video quality index.

Similar to the SSIM index, the video-SSIM is a FR quality assessment algorithm. The algorithm operates on the video in a frame-by-frame fashion. video-SSIM starts by calculating the SSIM index for a set of randomly chosen $8 \times 8$ windows. This is different from the original SSIM algorithm, which uses a sliding window approach to 
evaluate all possible windows in the image. As indicated in [19], a careful selection of the number of random sampling windows used $\left(R_{s}\right)$ can significantly reduce the algorithm computation time, while having little effect on the algorithm robustness. The video-SSIM algorithm operates in the YCbCr color space. The SSIM algorithm is applied to each color component of each sampling window independently and then combined into a local SSIM index using a weighted summation. Let SSIM $\underset{i j}{Y}, \operatorname{SSIM}_{i j}^{C b}$ and SSIM $_{i j}^{C r}$ be the local SSIM values for the $\mathrm{Y}, \mathrm{Cb}$ and $\mathrm{Cr}$ components of the $j$-th window in the $i$-th frame, respectiviely, then the local SSIm value is given by:

$$
S S I M_{i j}=W_{Y} S S I M_{i j}^{Y}+W_{C b} S S I M_{i j}^{C b}+W_{C r} S S I M_{i j}^{C r}
$$

where $W_{Y}, W_{C b}$ and $W_{C r}$ are weights. The weights were set to 0.8 for the luminance (Y) component and 0.1 for each of the chrominance components. Setting the luminance weight to 1 and 0 for chrominance weights makes the algorithm a gray scale video assessment algorithm.

To obtain the overall frame's SSIM index, the local window's SSIM values are weighted based on the average luminance (the Y component) of each window. SSIM values from windows with high luminance average are given a larger weight value than those with low average luminance (dark regions). This is because the HVS's attention is directed more towards bright areas than to dark ones when viewing a video sequence. The quality $Q_{i}$ of the $i^{\text {th }}$ frame is taken as

$$
Q_{i}=\frac{\sum_{j=1}^{R_{s}} w_{i j} S S I M_{i j}}{\sum_{j=1}^{R_{s}} w_{i j}}
$$

where $R_{s}$ is the number of local sampling windows in the frame and $w_{i j}$ is the weight used with the SSIM value of the $j^{\text {th }}$ window in the $i^{\text {th }}$ frame. Although more complex HVS derived weights can be used, [19] used the local luminance mean of the window 
$\left(\mu_{i j}\right)$, as described in (3.1), and derived the local weights using

$$
w_{i j}= \begin{cases}0, & \mu_{i j} \leq 40 \\ \left(\mu_{i j}-40\right) / 10, & 40<\mu_{i j} \leq 50 \\ 1, & \mu_{i j}>50\end{cases}
$$

To obtain the video sequence's quality, the frames' quality indices are weighted based on the amount of motion in each frame, before combining them in a single value. The motion based weights were used since the HVS is more tolerant to artifacts in fast moving objects. The overall video-SSIM index is calculated as

$$
\text { Video-SSIM }=\frac{\sum_{i=1}^{F} W_{i} Q_{i}}{\sum_{i=1}^{F} W_{i}}
$$

where $F$ is the number of frames used, $Q_{i}$ the $i^{\text {th }}$ frame's quality given by (3.14) and $W_{i}$ is the motion based quality weight of the $i^{\text {th }}$ frame.

To calculate $W_{i}$, the average motion of the frame is estimated first from all the local sampling windows' motion vectors. Let $m_{i j}$ be the motion vector length of the $j^{\text {th }}$ sampling window in the $i^{\text {th }}$ frame, then the motion level of the $i^{\text {th }}$ frame is estimated as

$$
M_{i}=\frac{\left(\sum_{j=1}^{R_{s}} m_{i j}\right) / R_{s}}{K_{M}}
$$

where $K_{M}$ is a constant used for normalizing the frame's motion level. $K_{M}$ was set to 16 in [19]. Using the motion level of each frame, the motion based frame quality weight $W_{i}$ can be calculated using 


$$
W_{i}= \begin{cases}\sum_{j=1}^{R_{s}} w_{i j}, & M_{i} \leq 0.8 \\ \left(\left(1.2-M_{i}\right) / 0.4\right) \sum_{j=1}^{R_{s}} m_{i j}, & 0.8<M_{i} \leq 1.2 \\ 0, & M_{i}>1.2\end{cases}
$$

Although the video-SSIM algorithm has good correlation values with subjective scores when tested against the VQEG Phase-I video sequences, it must be clarified that the algorithm does not account for temporal artifacts. The motion information estimated from the video sequence's frames is only used for weighting the quality index of each frame when combined into the final video quality. If the test video sequence had temporal errors such as multiple repeated frames replacing a fast motion segment of the video sequence, then the motion derived weights from those frame will reduce or even discard the effect of that distortion on the overall video quality. Also, since the video-SSIM algorithm uses the spatial SSIM algorithm for local window quality estimation, the problems of the SSIM algorithm sdiscussed previously are inherited.

Many research efforts worked in developing video quality assessment algorithms based on SSIM. Recent work by Lin and Chau [21] proposed using a hierarchal version of the SSIM index in the video-SSIM. The hierarchal SSIM was proposed to allow the video-SSIM to better detect spatial artifacts normally present in low-bit rate video system. Another algorithm that was motivated by the SSIM index is the V-SSIM algorithm proposed in [2]. V-SSIM decomposes the video sequence using a family of Gabor filters and incorporates motion models in its video quality evaluation. The quality index is calculated in the complex frequency domain and bases its quality assessment on the CW-SSIM algorithm. The algorithm was detailed in Sec. 2.7.8 of this document. 


\section{Chapter 4}

\section{Dual-Tree Complex Wavelet Transform}

\subsection{Wavelet Transform}

The wavelet transform (WT) is another algorithm used to transform a signal from the time domain to a frequency-like domain. Like the Fourier transform (FT) and the discrete cosine transform (DCT), the WT is used for signal analysis, design, digital communication and processing.

Unlike the FT and the DCT, the WT provides us with good time-frequency localization. Also, by its nature and the way it is constructed, the WT yields a multiresolution signal analysis. The basis used in the WT, $\Psi(t)$, can be any function that represents a compact oscillatory wave which satisfies both conditions:

$$
\int_{-\infty}^{\infty} \Psi(t) d t=0
$$

and

$$
\int_{-\infty}^{\infty}|\Psi(t)|^{2} d t<\infty
$$

Once a basis function is selected as the transform's basis, the continuous wavelet transform (CWT) of a function $f(t)$ at a scaling factor $s$ and translation $\tau$, denoted 
as $W(s, \tau)$, can be calculated as follows

$$
W(s, \tau)=\int_{-\infty}^{\infty} f(t) \Psi_{s, \tau}^{*}(t) d t
$$

where $\Psi_{s, \tau}^{*}(t)$ is the complex conjugate of the scaled and translated wavelet basis obtained from the mother wavelet as

$$
\Psi_{s, \tau}(t)=\frac{1}{\sqrt{s}} \Psi\left(\frac{t-\tau}{s}\right)
$$

Because the transform is calculated by continuously shifting a continually scaled function over the signal, the coefficients from the transform are highly redundant. To make computation more practical, the discrete wavelet transform (DWT), obtained by sampling the CWT, is used. In practice, there is no need to explicitly compute the shape of the wavelets and the scaling functions. The transform is carried out by designing a filter bank using the coefficients to obtain perfect reconstruction low and high pass filters. With a special choice of the mother wavelet, the discrete wavelets are made orthogonal to dilations and translations. Orthogonality is not an essential condition in constructing the wavelet transform. However, imposing the orthogonality condition on the selection of the wavelet allows us to obtain our filter bank from a single low-pass filter $h(t)$. The filter bank will consist of the low-pass $h(t)$ filter and a corresponding high-pass filter $g(t)$. Two more reconstruction filters $(\hat{h}(t)$ and $\hat{g}(t))$ for signal synthesis are obtained from the low and high pass filters used for signal analysis. The low-pass filter will output the approximation of the signal and the high-pass will provide us with the details lost between approximations. Multi-resolution analysis is obtained by applying the filter bank in a recursive fashion to the approximations from the previous pass with the number of iterations depending on the application. 

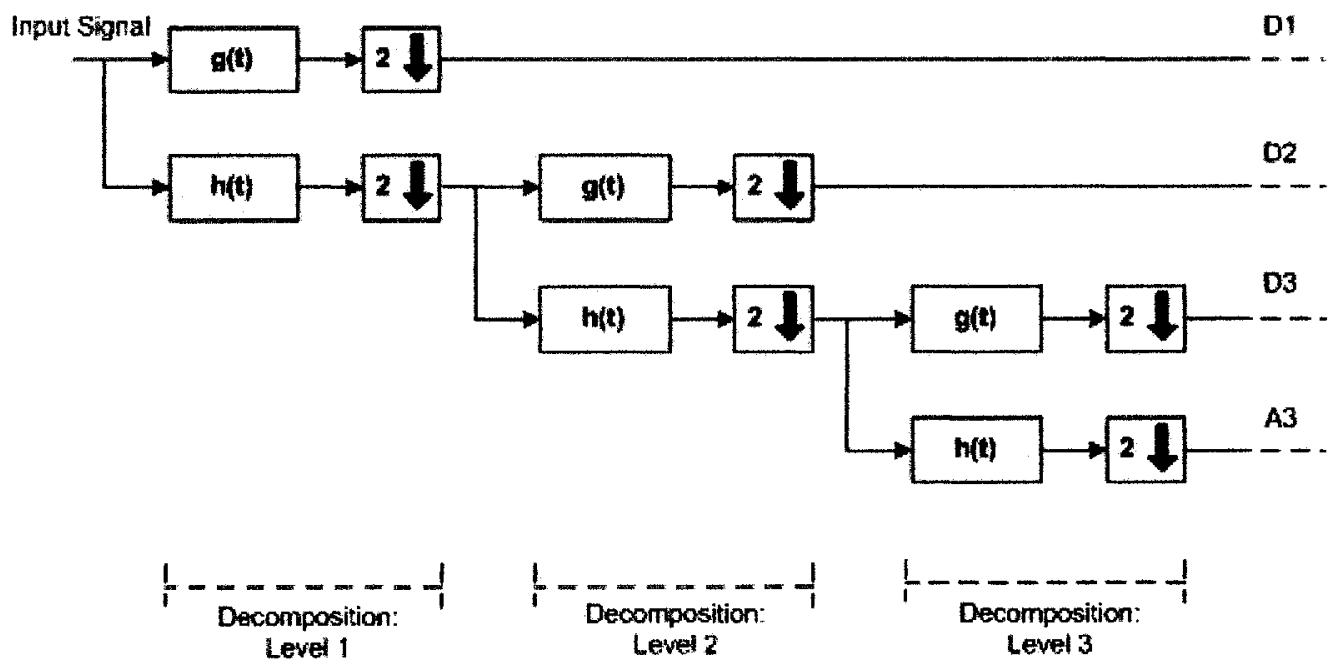

(a) Signal analysis

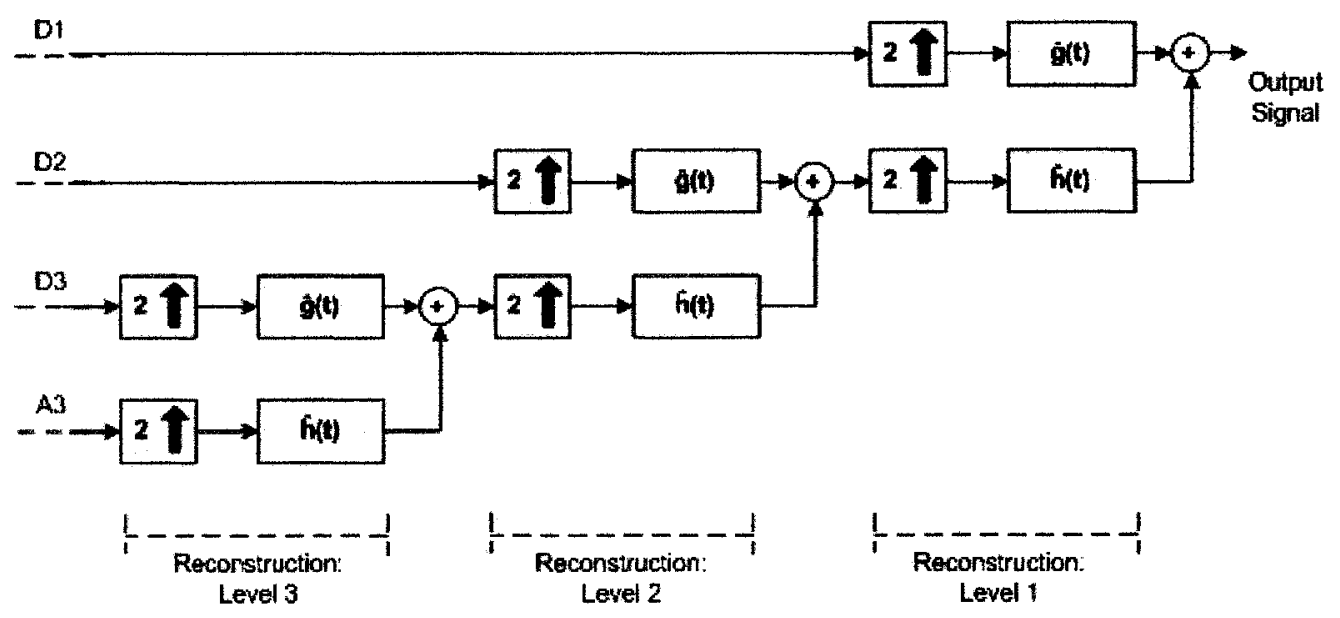

(b) signal synthesis

Figure 4.1: Multi-scale discrete wavelet transform using a wavelet filter bank, where $\mathrm{A} n$ and $\mathrm{D} n$ are the approximation and detail coefficients from decomposition level $n$, respectively. (a) signal decomposition/analysis, (b) signal reconstruction/synthesis. 
The transform using an analysis and synthesis filter bank is shown in Figure 4.1.

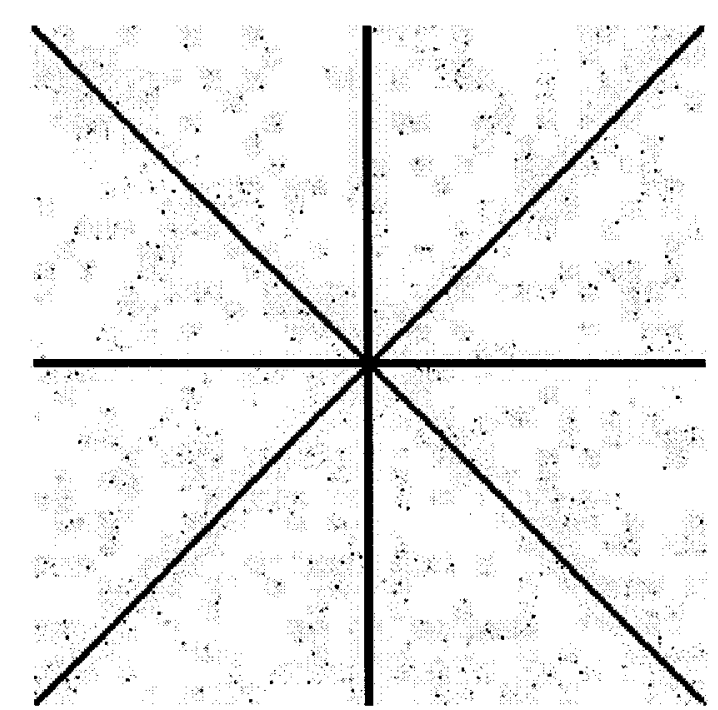

(a)

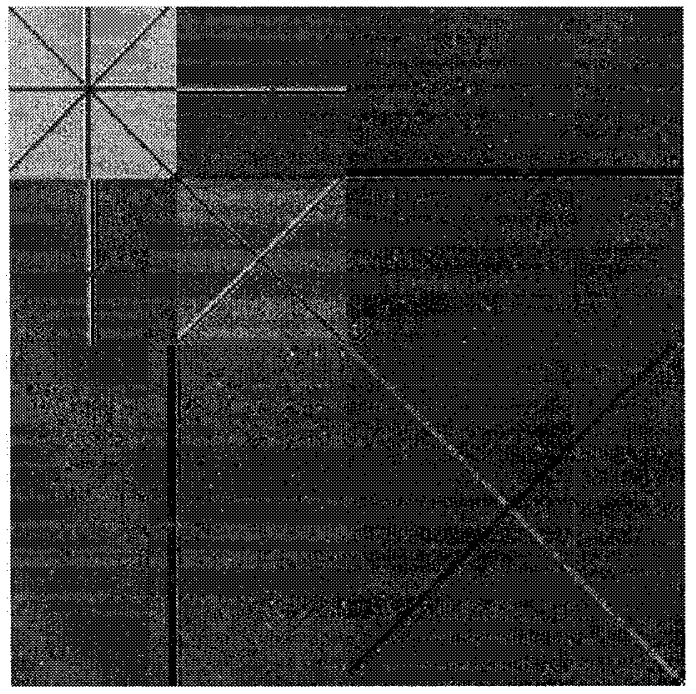

(b)

Figure 4.2: 2 level DWT image decomposition. (a) is the original star image and (b) is the 2-scale DWT decomposition bands from the star image.

Note that: The DWT was carried on using the Daubechies 8 tap wavelet. Also, the image coefficients' bands were normalized individually, for better visibility, before being organized and displayed

The above discussion is for the 1-D DWT. However, higher dimension transforms can be obtained by applying the transform in each dimension in a separable fashion. The 2-D transform is achieved by filtering the signal horizontally then vertically by the same filter bank. To perform a 2-D DWT, the 2-D signal's rows are filtered with the low- and high-pass filters, and then down-sampled vertically resulting in a low frequency band (L) and a high frequency band $(\mathrm{H})$. Both the $(\mathrm{L})$ and $(\mathrm{H})$ bands are then filtered horizontally with the same filter bank used before then down-sampled horizontally. The horizontal filtering yields (LL) and (LH) bands from the (L) band and $(\mathrm{HL})$ and $(\mathrm{HH})$ bands from the $(\mathrm{H})$ band. A single level wavelet transform on a 2-D signal will yield four coefficient sets/subbands, an approximation subband (LL) and a vertical (HL), horizontal (LH) and diagonal (HH) detail subbands. Figure 4.2 
shows a 2 scale decomposition of the star image. Notice the strong horizontal and vertical orientation edges present in the horizontal and vertical bands. Also, note that the approximation band represents a smaller, somewhat blurred, version of the original image.

\subsection{Dual-Tree Complex Wavelet Transform}

The dual-tree complex wavelet transform $(\mathrm{DT}-\mathbb{C W T}$ ) is a relatively new wavelet transform [22], designed with a few shortcomings of the DWT in mind. Unlike the DWT, the DT-CWT is designed to be almost shift invariant with good direction selectivity [22,71-74]. The strong directional orientation of the DT-CWT comes from its ability to separate positive from negative frequencies into different bands. Similar to steerable filters, the DT-CWT is an overcomplete wavelet transform with a limited redundancy of $2^{d}$, where $d$ is the number of dimensions in the processed signal [22].

In [71], Nick Kingsbury provides a method to design complex filters, which through the use of them and their complex conjugates, the processed signal can be fully represented. Later in [72], Kingsbury provided Daubechies-like filters that had smaller support, making the transform more feasible than what was presented in [71]. In [73] and [74], Selensnick discussed Kingsbury's work and was able to derive the DT-CWT filters as a Hilbert transform pair, simplifying their design process.

The DT-CW' is designed with two wavelet bases in a Daubechies-like form. The bases $\Psi_{h}(t)$ and $\Psi_{g}(t)$ must form a Hilbert transform pair $\Psi_{g}(t)=\mathcal{H}\left(\Psi_{h}(t)\right)$, to overcome the oscillation, aliasing, shift variance, and lack of directionality problems associated with the DWT as outlined in [22]. Both wavelet bases are real and yield their own set of coefficient bands. However, $\Psi_{h}(t)$ is taken as the real part of the 
transform and $\Psi_{g}(t)$ is treated as the imaginary part, making the overall basis as $\Psi(t)=\Psi_{h}(t)+j \Psi_{g}(t)[22]$. Figure 4.3 shows a filter bank representation of the 1-D DT-CWT.

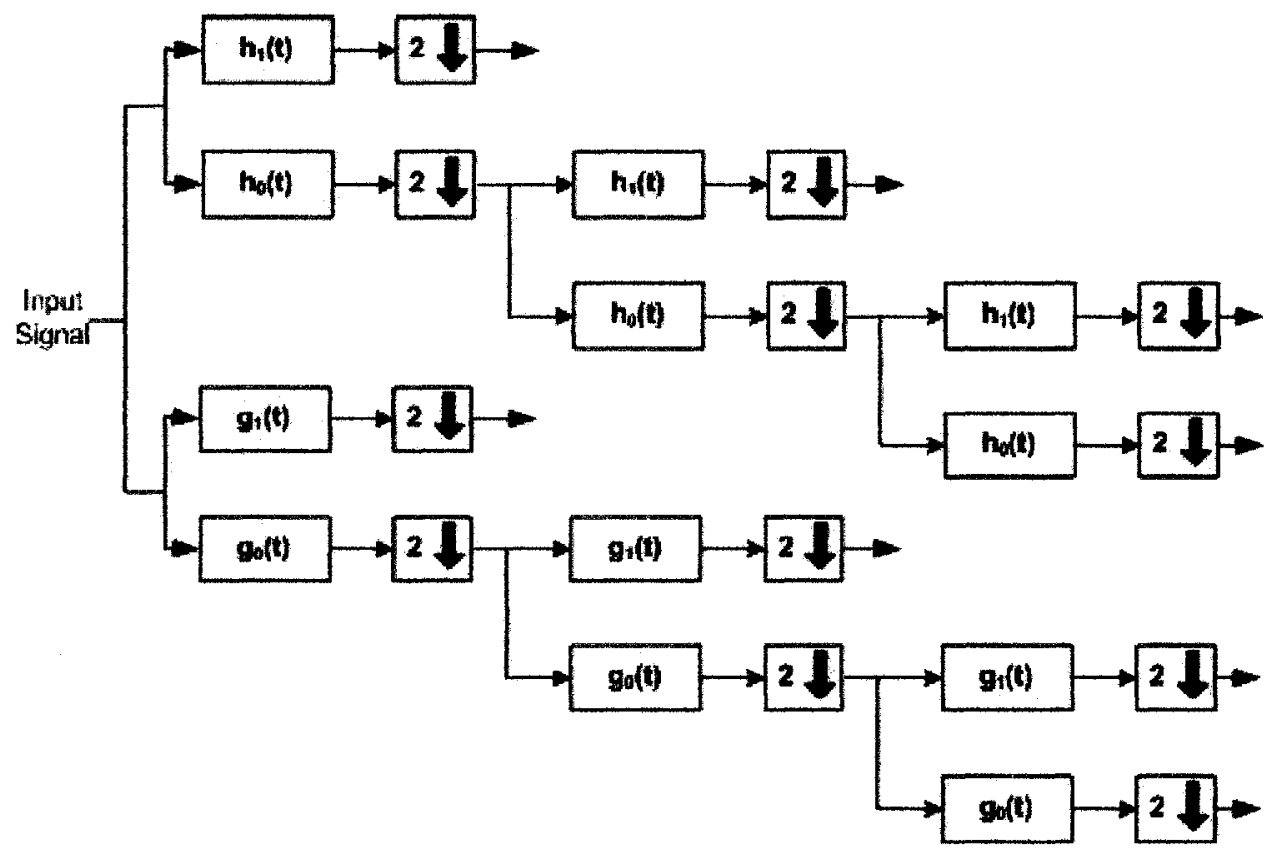

Figure 4.3: Dual-tree complex wavelet analysis filter banks with three levels of decomposition. The signal is transformed by both sets of filters. Each filter bank yields its own set of real coefficients. However, the complex coefficients are achieved by treating one filter bank's output as the real part of the transform and the other as the imaginary part.

By extending the DT-CWT transform to higher dimensions, direction selectivity is added to the transform's near shift invariance property. In 2-D, the transform is able to separate the different edge orientations into different subbands. The 3-D DT-CWT extends this property to he $3-\mathrm{D}$ space. When used with video signals, the 3-D DT-CWT is seen as a motion selective transform, separating different orientation and motion directions into different subbands.

Using a higher dimension DT-CWT can be more attractive than using a separable DWT of the same dimension. This is because the DT-CWT provides non-separable 
wavelets, by linearly combining different separable wavelets, while maintaining the simplicity of a separable transform. Another attractive feature of higher dimensions DT-CWT is the lack of the checkerboard artifact that is observed in the separable DWT. The checkerboard artifact, which results from mixing different directions in one subband, is a serious analytical problem that is elevated as the transform's dimension is increased. The DT-CWT eliminates the checkerboard artifact by separating positive from negative frequencies into different subbands.

To obtain the different orientation wavelets associated with the 2-D DT-CWT, we start by defining the row-column diagonal band (high-high) 2-D wavelet $\Psi(x, y)=$ $\Psi(x) \Psi(y)$. Using $\Psi(t)=\Psi_{h}(t)+j \Psi_{g}(t)$, the 2-D wavelet is expressed as

$$
\Psi(x, y)=\Psi_{h}(x) \Psi_{h}(y)-\Psi_{g}(x) \Psi_{g}(y)+j\left[\Psi_{g}(x) \Psi_{h}(y)+\Psi_{h}(x) \Psi_{g}(y)\right]
$$

With $\Psi_{g}(t)=\mathcal{H}\left(\Psi_{h}(t)\right)$, the real part of this wavelet

$$
\begin{aligned}
\Psi_{r} & =\Re e\{\Psi(x, y)\} \\
& =\Psi_{h}(x) \Psi_{h}(y)-\Psi_{g}(x) \Psi_{g}(y)
\end{aligned}
$$

has a symmetric spectrum capturing only one diagonal direction. For the other diagonal direction, we take $\Psi_{2}(x, y)=\Psi(x) \overline{\Psi(y)}$, where the over line indicates the complex conjugate. With these two wavelets, the 2-D DT-CWT replaces the DWT's diagonal wavelet with two wavelets, each with its own distinct orientation, eliminating the checkerboard artifact present in that subband.

Repeating the process for the other two DWT subbands using; $\Phi(x) \Psi(y)$, $\Psi(x) \Phi(y), \Phi(x) \overline{\Psi(y)}$, and $\Psi(x) \overline{\Phi(y)}$, where $\Phi(t)$ is the scaling function expressed 


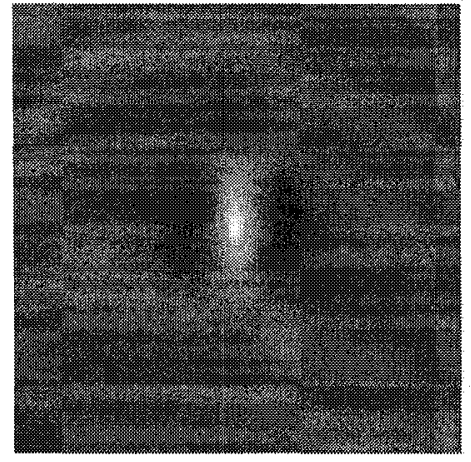

(a)

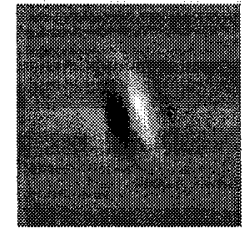

(d)

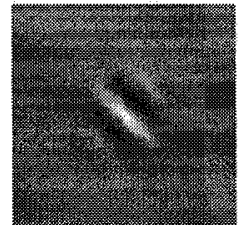

(e)

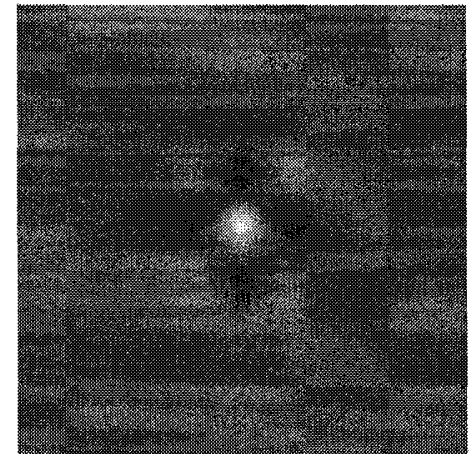

(b)

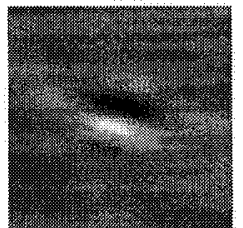

(f)

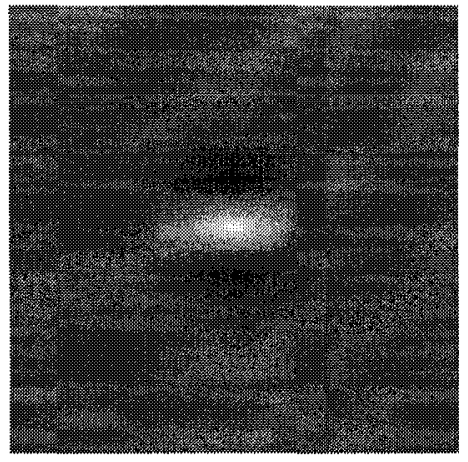

(c)

Figure 4.4: Typical 2-D wavelet bases. ${ }^{a}$ (a)-(c) are the 3 wavelets associated with the separable DWT. (d)-(i) are the 6 real wavelet basis associated with the DT-CWT.

Note the checkerboard artifact visible in the diagonal wavelet of the DWT shown in (b). The DT-CWT eliminates this problem by representing the diagonal subband with the two wavelets shown in $(e)$ and $(h)$.

${ }^{a}$ The different wavelet bases were generated using the MATLAB code available from [75].

as $\Phi(t)=\Phi_{h}(t)+j \Phi_{g}(t)$, adds four more orientation wavelets to the two we already have. With that, the DT-CWT replaces the three DWT wavelets with a total of six distinct non-separable 2-D orientation wavelets. The different wavelets associated with the 2-D DWT and the 2-D DT-CWT are shown in Figure 4.4. Each one of the six 2-D non-separable DT-CWT wavelets can be expressed as a linear combination of two separable $2-\mathrm{D}$ wavelet basis, using

$$
\begin{array}{r}
\Psi_{i}(x, y)=\Psi_{1, i}(x, y)-\Psi_{2, i}(x, y) \\
\Psi_{i+3}(x, y)=\Psi_{1, i}(x, y)-\Psi_{2, i}(x, y)
\end{array}
$$


for $i=1,2,3$, where the six separable 2-D wavelet bases are given by

$$
\begin{aligned}
& \Psi_{1,1}(x, y)=\Phi_{h}(x) \Psi_{h}(y), \quad \Psi_{2,1}(x, y)=\Phi_{g}(x) \Psi_{g}(y), \\
& \Psi_{1,2}(x, y)=\Psi_{h}(x) \Phi_{h}(y), \quad \Psi_{2,2}(x, y)=\Psi_{g}(x) \Phi_{g}(y), \\
& \Psi_{1,3}(x, y)=\Psi_{h}(x) \Psi_{h}(y), \quad \Psi_{2,3}(x, y)=\Psi_{g}(x) \Psi_{g}(y) .
\end{aligned}
$$

The DT-CWT can be extended to 3-D in a similar fashion to that of the 2-D extension. Using 3-D DT-CWT, the diagonal 3-D separable DWT subband (highhigh-high) is represented by first defining $\Psi(x, y, t)=\Psi(x) \Psi(y) \Psi(t)[76]$, with its real part represented as

$$
\begin{aligned}
\Psi_{r} & =\Re e\{\Psi(x, y, t)\} \\
& =\Psi_{a}(x, y, t)-\Psi_{b}(x, y, t)-\Psi_{c}(x, y, t)-\Psi_{d}(x, y, t)
\end{aligned}
$$

with

$$
\begin{aligned}
& \Psi_{a}(x, y, t)=\Psi_{h}(x) \Psi_{h}(y) \Psi_{h}(t), \\
& \Psi_{b}(x, y, t)=\Psi_{g}(x) \Psi_{g}(y) \Psi_{h}(t), \\
& \Psi_{c}(x, y, t)=\Psi_{g}(x) \Psi_{h}(y) \Psi_{g}(t), \\
& \Psi_{d}(x, y, t)=\Psi_{h}(x) \Psi_{g}(y) \Psi_{g}(t) .
\end{aligned}
$$

In 3-D, unlike the 2-D extension, four separable wavelet bases are linearly combined to give us a single non-separable 3-D DT-CWT wavelet that has a distinct orientation in space. To capture the other orientations present in the diagonal 3-D DWT subband, we define; $\Psi_{2}(x, y, t)=\Psi(x) \Psi(y) \overline{\Psi(t)}, \Psi_{3}(x, y, t)=\Psi(x) \overline{\Psi(y)} \Psi(t)$, and $\Psi_{4}(x, y, t)=\Psi(x) \overline{\Psi(y) \Psi(t)}$. With the 3-D DT-CWT, each one of the DWT's 7 subbands is replaced by 4 subbands, each with its own orientation and motion 
selectivity, to a total of 28 distinct subbands. See Figure 4.5 for 3-D wavelet basis examples. For a full detailed discussion regarding the 2-D and 3-D DT-CWT, see $[22,76,77]$. 


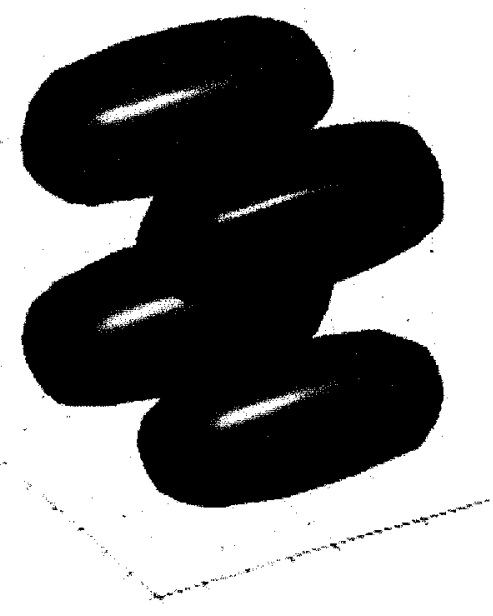

(a)

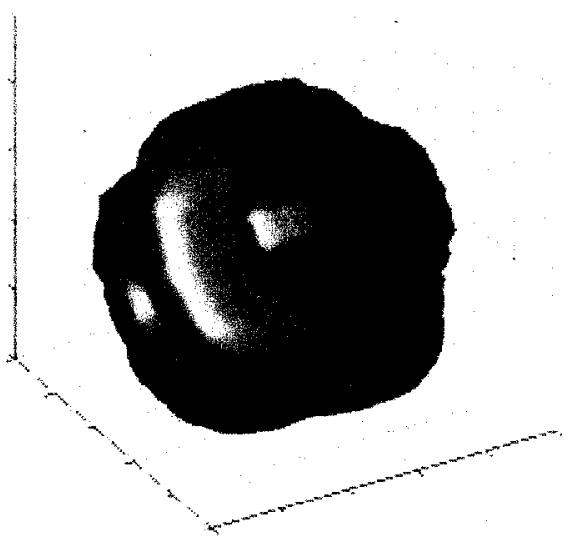

(c)

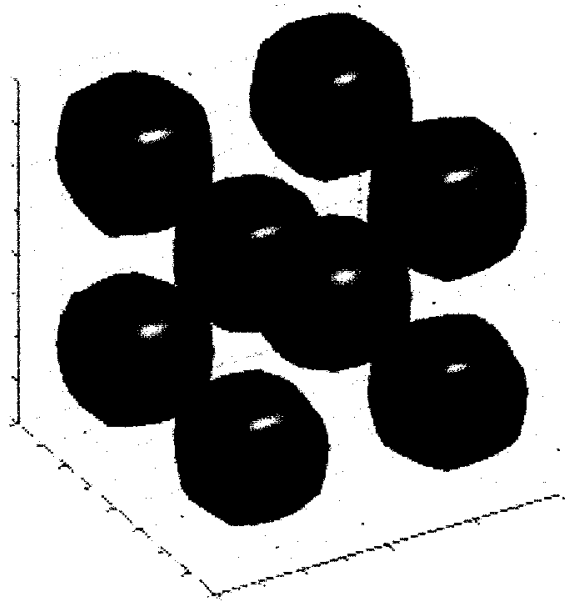

(b)

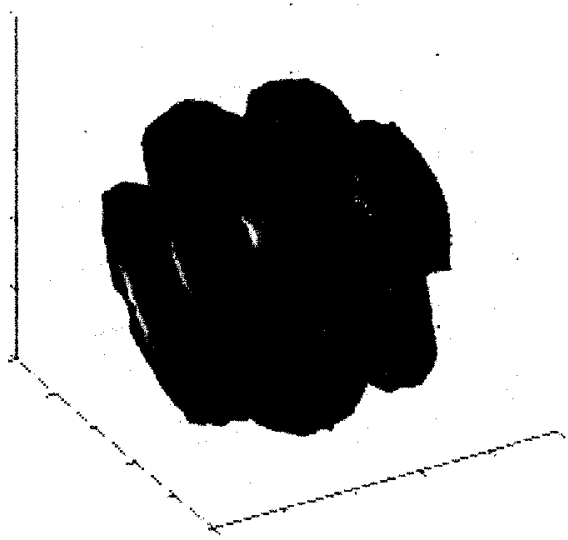

(d)

Figure 4.5: typical 3-D wavelet bases $^{a}$.(a) and (b) are the 2 of the 7 wavelets associated with the separable 3-D DWT. (c) and (d) are 2 of the 28 real wavelet basis associated with the 3-D DT-CWT.

Note the good orientation present in the 3-D DT-CWT wavelets.

\footnotetext{
The 3D wavelets were generated using the DT wavelet code from [75]
} 


\section{Chapter 5}

\section{Image Quality Assessment using the Dual-Tree Complex Wavelet SSIM}

There is a constant need for objective video quality algorithms that can evaluate the performance of video processing algorithms as well as assist in their development. To achieve good quality prediction, many quality assessment algorithms were developed based on the HVS's characteristics (see Chapter 2). However, such implementations result in complex assessment algorithms. Looking at the HVS from a new perspective, Wang et al. developed the SSIM index which has good quality prediction although simple to implement [3]. In recent years, different video applications have been developed successfully using the newly developed DT-CWT. With that in mind, we decided to implement a video quality assessment tool that is based on the CW-SSIM algorithm and uses the DT-CWT at its core.

The CW-SSIM is reported to perform better than its original spatial implementation, the SSIM index. Also, many publications show assessment results obtained using both the SSIM and CW-SSIM algorithms, discussing how the CW-SSIM results agree more with the amount of error visible in the used test images. However, we 
could not find any work that provides a full performance comparison, using a subjectively scored image set, between the two algorithms. For that reason, and before we work in the video domain, in this chapter we evaluate the performance of the CW-SSIM algorithm to validate its suitability. Also, we evaluate the performance of the algorithm with the DT-CWT on images, which hasn't yet been done in the literature, before applying it to video sequences.

\subsection{Image Quality Assessment in the Dual-Tree Domain}

It is highly desirable for quality assessment to have an algorithm that is simple to implement, has high performance, and has a clear physical meaning. Furthermore, the ability to embed the quality algorithm in image and/or video processing algorithms, for optimization and quality control, elevates the usefulness of the quality algorithm.

With the development of wavelet based compression algorithms, different wavelet based quality assessment techniques were developed, see Sec. 2.6.8 and Sec. 2.6.9. As such quality algorithms share the same transforms as the wavelet compression algorithms, they can be incorporated as part of the wavelet compression to enhance their performance.

The $\mathbb{C W}$-SSIM algorithm was developed to maintain the desired characteristics of the SSIM algorithm, while lowering the algorithm's sensitivity to geometric distortions. Although the algorithm is developed to operate using any complex wavelet transform, the literature only shows implementations using the steerable pyramid (SP) transform $[5,6,20,78]$. The SP transform decomposes signals into different reso-

lution bands, each with a number of distinct orientation subbands [62,79]. However, 
the SP filter design can be messy. Also, the space-domain implementation is not perfect reconstruction, and the perfect reconstruction frequency domain implementation exhibits spatial ringing [80].

The DT-CWT, although a fairly new transform, has been successfully used in different applications such as image and video denoising $[22,77]$ and image and video compression $[76,81,82]$. Also, the DT-CWT has simpler filter design and lower redundancy than that of the SP transform [71]. With that in mind, we propose implementing the $\mathbb{C W}$-SSIM algorithm using the DT-CWT. Using the DT-CWT as opposed to the SP transform should allow the algorithm to

- be embedded into dual-tree based image denoising, image coding and noise shaping algorithms, for optimization and quality control; and

- be extended for video assessment since the DT-CWT is extendable as a 3-D transform.

For this implementation, we will only substitute the SP transform with the DT$\mathbb{C W T}$ and will not change the algorithm's overall design. We will refer to this implementation as the DT-CW-SSIM and to the original implementation, using the SP transform, as SP-CW-SSIM.

\subsection{LIVE Image Database}

The $\mathbb{C W}$-SSIM was developed to enhance the performance of the SSIM algorithm. However, our literature survey found no published work that properly verified the performance enhancement gain from working in the complex transform domain. For that reason, we wanted to test the performance of the $\mathbb{C W}$-SSIM algorithm before using it in a 3-D video context. 
To be able to verify the $\mathbb{C W}$-SSIM algorithm's performance and compare it with that of the SSIM index, we required a scored image database that contains a variety of image information and a number of different image distortions.

For our algorithm evaluation tests, we used the release 2 images of the LIVE image quality assessment database [83]. The database consists of 982 subjectively scored images created by distorting 29 high-resolution reference images with a variety of distortions at different levels. The reference images are 24-bit/pixel RGB color images, with thumbnails of the database's reference images shown in Figure 5.1. The distortions used to build the database are JPEG2000 compression, JPEG compression, White Noise, Gaussian Blur, and transmission errors in the JPEG2000 bit stream using a fast-fading Rayleigh channel model. For more information about the LIVE quality assessment database see [83].

To test the different algorithms we used 2 collections of test images, Coll-I and Coll-II, constructed from the images in the LIVE database. The images in Coll-I contain only the JPEG and JPEG2000 compressed images. We used this collection as a performance evaluation of the different algorithms against compression related artifacts. The images in Coll-II are simply the full image set from the LIVE database. Using Coll-II allows for a full performance evaluation of the different algorithms against a varied range of distortion types and levels,

It is important to note here that the LIVE image database does not contain images with geometric distortions. However, as the reduced sensitivity of the CW-SSIM algorithm to geometric artifacts was discussed and compared with that of the SSIM algorithm in [20], the lack of geometrically distorted images will not negatively affect our algorithm performance evaluation. With no geometrically distorted images in the testbed, our performance evaluation will highlight any performance enhancements gained by moving the SSIM algorithm to the complex wavelet domain, aside from 
that gained by reducing its sensitivity to small geometric artifacts.

\subsection{Implementations and Results}

To verify the performance of the $\mathbb{C W}$-SSIM algorithm and to test the effect of replacing the SP transform, initially used with the algorithm, with the DT-CWT, we implemented the CW-SSIM using both transforms.

For both DT-CW-SSIM and SP-CW-SSIM implementations, we used the DTCWT and the SP transform at four dyadic levels of multiresolution decomposition with six orientations each. This decomposition map of both transforms is compliant with the number of resolution levels and orientation subbands of the HVS (see Sec. 2.1.1). Most of the structure information is extracted into the detail subbands of both SP and DT-CWT leaving a blurred down-sampled version of the image in the lowpass subband. With that in mind, we decided to use only the detail subbands in our implementations of the CW-SSIM algorithms.

Another $\mathbb{C W}$-SSIM implementation that we tested used the SP transform with 16 orientation subbands for each decomposition. We included this number of orientation in our testing as it was the number used in [20] to initially test the algorithm and later used in [6]. Also, as this implementation contains a higher number of distinct orientation subbands, we wanted to test the effect the number of orientation bands on the algorithms quality prediction. To distinguish this test from the SP-CW-SSIM implementation, we will refer to this version as SP-CW-SSIM 2 .

Since the image database, used for the algorithm evaluation, is composed of color images, the algorithm will first transform the images from their RGB color space to the $\mathrm{YCbCr}$ color space, resulting in a luminance $(\mathrm{Y})$ and two chrominance $(\mathrm{Cb}$ and Cr) images. After that, the CW-SSIM algorithm decomposes the luminance and the 


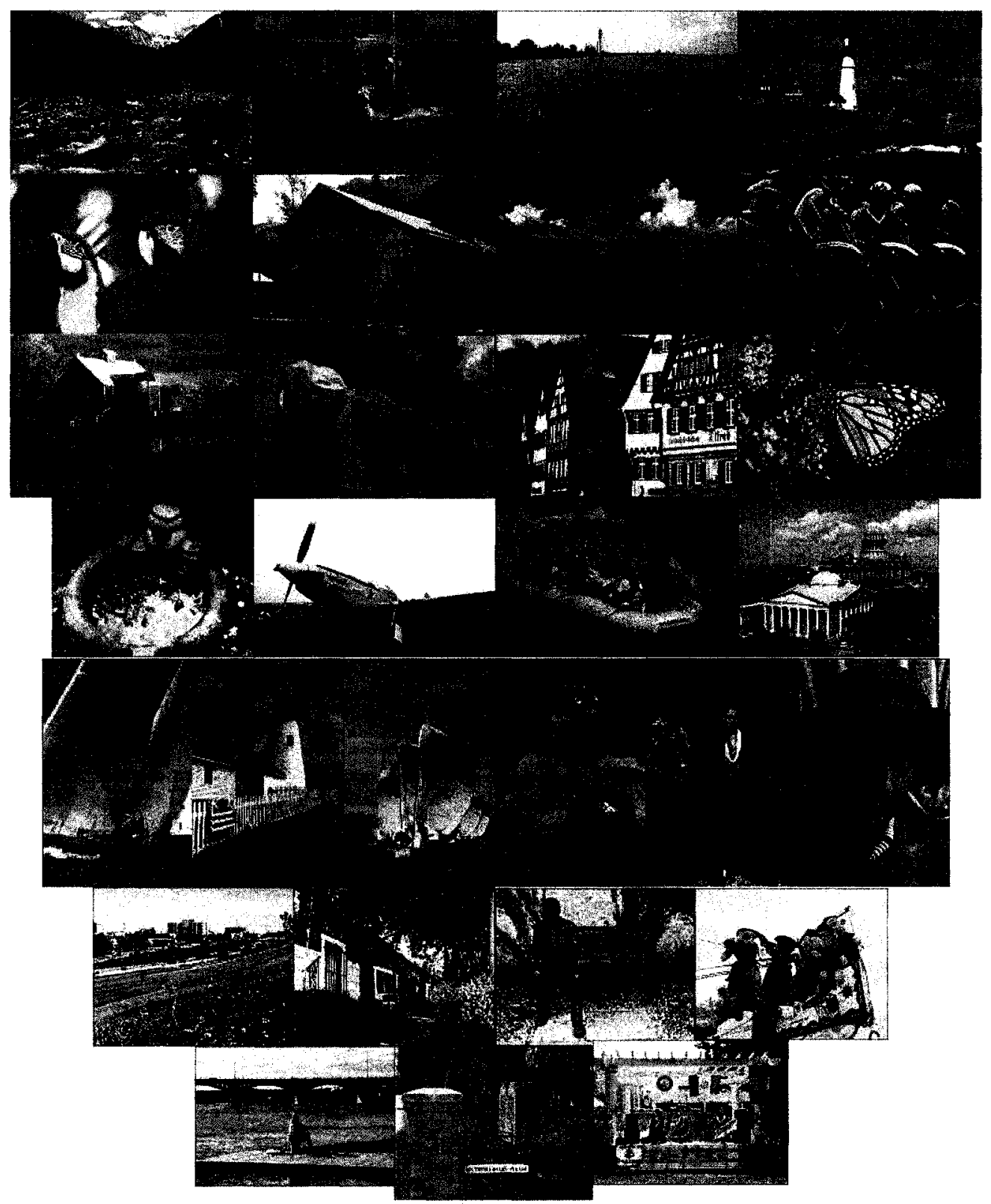

Figure 5.1: Thumbnails of LIVE database reference images. 
two chrominance images of both reference and test images, as described in [20], using the specified transform. Following the decomposition, the CW-SSIM is calculated locally for small subsets of the coefficients extracted from the different decomposition subbands. A $7 \times 7$ sliding window is used to extract coefficient subsets from the same spatial location in corresponding decomposition subbands of corresponding reference and test images. A recent study by Brooks et al. investigated the effect of the window size on the performance of the quality assessment. The study concluded that a window size of $7 \times 7$ yields results that correspond well with the human perception over a wide range of image distortions [6]. For each decomposition subband, the local windows' CW-SSIM values from the $\mathrm{Y}, \mathrm{Cb}$, and $\mathrm{Cr}$ images are combined as a weighted sum to obtain the color image's local window quality index. Each colored window quality index value $(Q)$ is taken as $Q=0.8 Q_{Y}+0.1 Q_{C b}+0.1 Q_{C r}$, where $Q_{Y}, Q_{C b}$ and $Q_{C r}$ are the $\mathbb{C W}-$ SSIM quality index for the corresponding local $7 \times 7$ windows of the same decomposition subband from the $\mathrm{Y}, \mathrm{CB}$, and $\mathrm{Cr}$ images, respectively. The resulting CW-SSIM values of the different sub-blocks are averaged similar to (3.10), used with the SSIM index, to obtain an orientation subband's quality index. Each level's orientation subband's quality indices are averaged to get the decomposition level's quality. The final quality index of the overall image is taken as the mean quality index of all the level's qualities, see Algorithm 1.

For our analysis, all structure similarity results, from the different algorithms, were converted to difference quality results (DQR). The DQR values are obtained by adjusting the quality index scale to match that of the DMOS values. This changes the quality index from having a highest quality of 1 and worst quality of 0 , to having a best quality when the DQR is 0 and a worst reported quality when the DQR is 


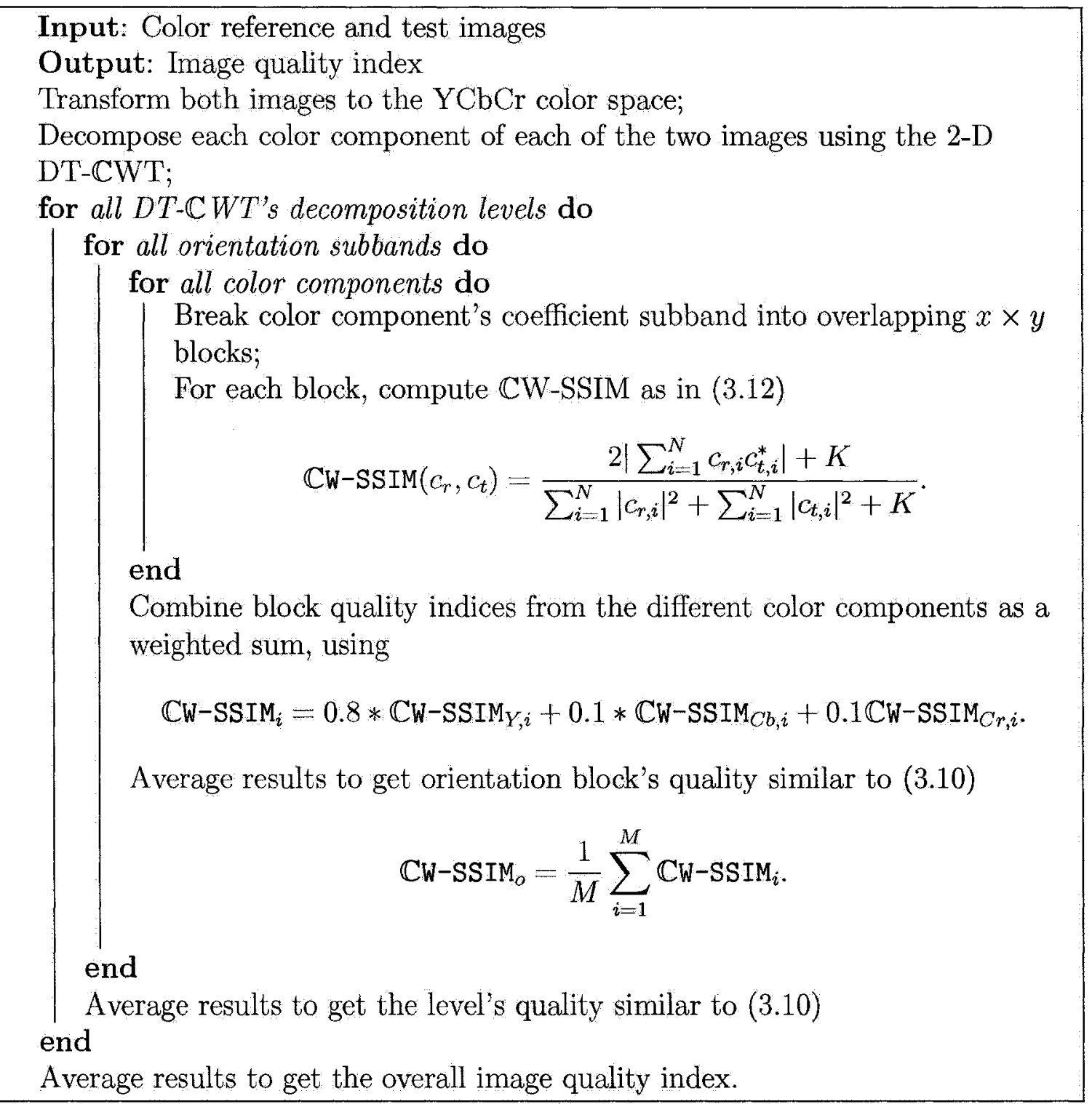

Algorithm 1: Image DT-CW-SSIM 
100. To be able to compare the performance of the three CW-SSIM implementations, we decided to calculate three correlation coefficients between the algorithms' DQR and the subjective database's DMOS values. The three coefficients used are: Pearson's linear correlation coefficient $(C C)$, Spearman's rank order correlation coefficient $(R O C C)$, and the correlation coefficient after transforming the results using non-linear regression $\left(C C_{2}\right) . C C_{2}$ is taken as Pearson's linear correlation coefficient between the set of predicted mean opinion scores $\left(\mathrm{DMOS}_{P}\right)$ and the viewer's DMOS values. The set of $\mathrm{DQR}$ results are transformed to a set of $\mathrm{DMOS}_{P}$ values through a nonlinear regression function which is fitted to the [DMOS, DQR] data set using the logistic function, given as

$$
\operatorname{DMOS}_{P}=\frac{b_{1}}{1-\exp \left(-b_{2}\left(\mathrm{DQR}-b_{3}\right)\right)}
$$

as discussed in the VQEG phase II report [41]. Both $C C$ and $C C_{2}$ are used to measure the model's prediction accuracy were the $R O C C$ is used to predict the model's monotonicity (the degree of agreement between the algorithm's prediction results and the subjective scores [41]).

All the test results from the different $\mathbb{C W}$-SSIM implementations were compared with PSNR and SSIM results from the same image sets. The different quality assessment algorithms were evaluated against the two test image collections described in Sec. 5.2. First, we evaluated the algorithms using images in Coll-I, which are the JPEG and JPEG2000 distorted images. The results of this test are presented in Table 5.1. Scatter plots of the objective DQR and the images' DMOS values are shown in Figure 5.2.

To evaluate the different algorithms against a more varied range of distortion types 


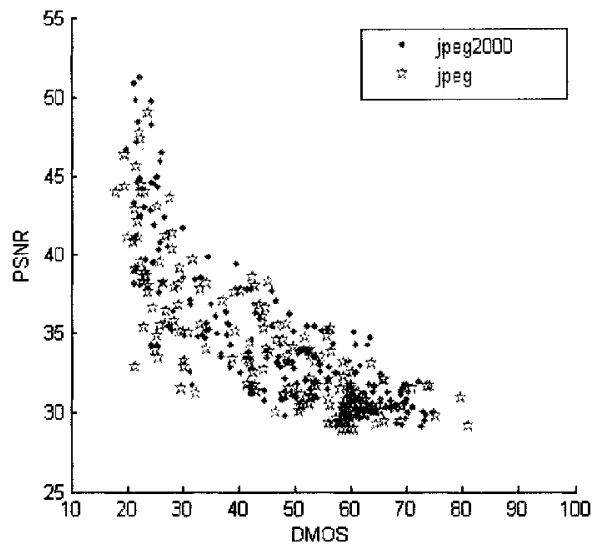

(a) PSNR

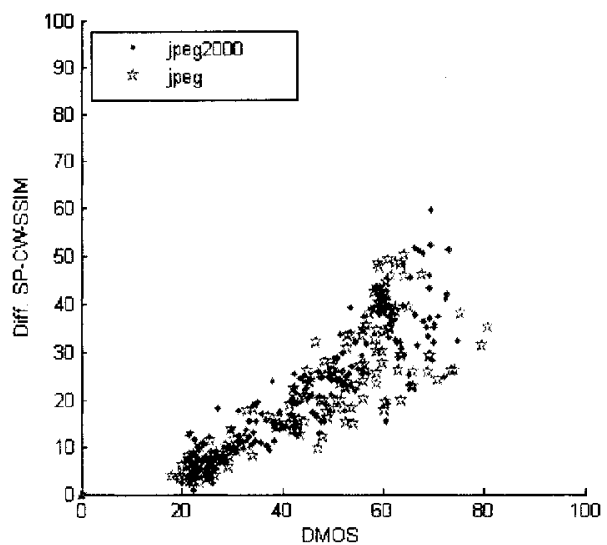

(c) SP-CW-SSIM

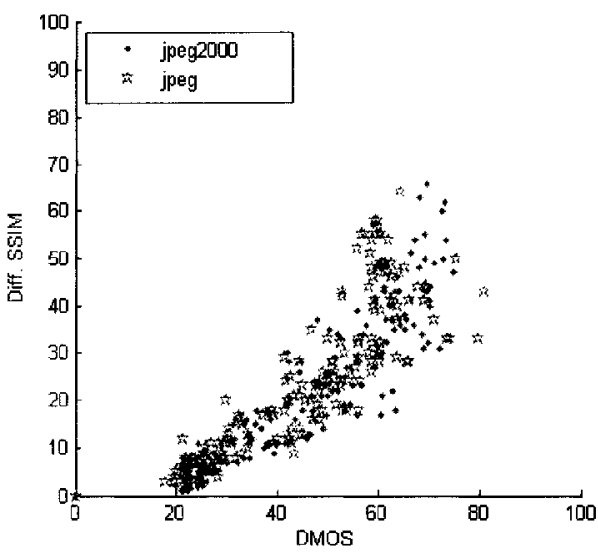

(b) SSIM

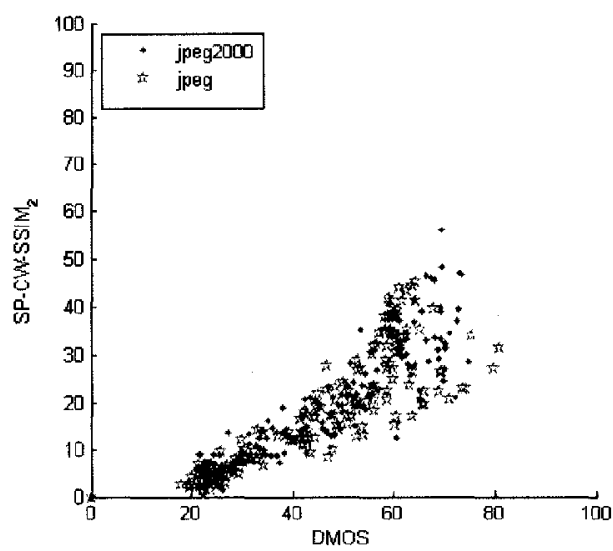

(d) SP-CW-SSIM

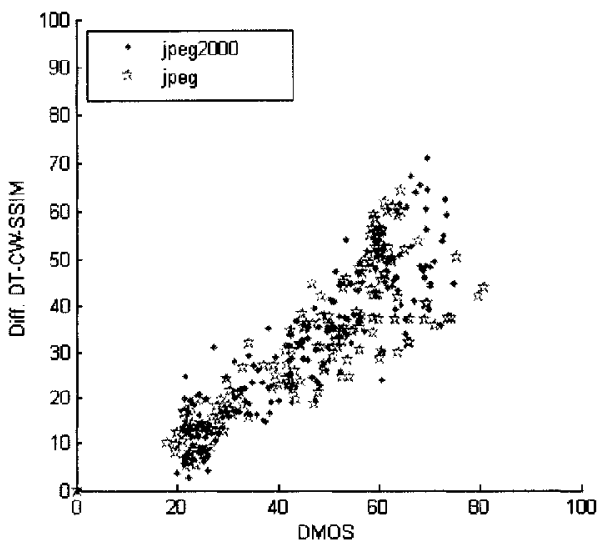

(e) DT-CW-SSIM

Figure 5.2: Scatter plots of the different difference quality results vs. the DMOS values for all the JPEG and JPEG2000 images in the LIVE image database. 
Table 5.1: Correlation coefficient $(C C)$, non-linear mapped correlation coefficient $\left(C C_{2}\right)$ and rank order correlation coefficient $(R O C C)$ of the objective quality results vs. DMOS for JPEG and JPEG2000 compressed images in release 2 of the LIVE image database.

\begin{tabular}{|c||ccc|}
\hline Objective test & $C C$ & $C C_{2}$ & $R O C C$ \\
\hline \hline PSNR & 0.8600 & 0.9116 & 0.9293 \\
SSIM & 0.8907 & 0.9495 & 0.9639 \\
SP-CW-SSIM & 0.9096 & 0.9559 & 0.9573 \\
SP-CW-SSIM & 0.9155 & 0.9539 & 0.9612 \\
DT-CW-SSIM & 0.9350 & 0.9570 & 0.9527 \\
\hline
\end{tabular}

Table 5.2: Correlation coefficient $(C C)$, non-linear mapped correlation coefficient $\left(C C_{2}\right)$ and rank order correlation coefficient $(R O C C)$ of the objective quality results vs. DMOS for all images in release 2 of the LIVE image database.

\begin{tabular}{|c||ccc|}
\hline Objective test & $C C$ & $C C_{2}$ & $R O C C$ \\
\hline \hline PSNR & 0.7883 & 0.8881 & 0.8930 \\
SSIM & 0.7506 & 0.9079 & 0.9040 \\
SP-CW-SSIM & 0.8262 & 0.9140 & 0.9334 \\
SP-CW-SSIM & 0.8124 & 0.9150 & 0.9234 \\
DT-CW-SSIM & 0.8866 & 0.9309 & 0.9378 \\
\hline
\end{tabular}

and levels, the images in $C o l l-I I$ were used. The results of this test are presented in Table 5.2. Figure 5.3 shows the scatter plots of the different algorithms' results against the DMOS values. 


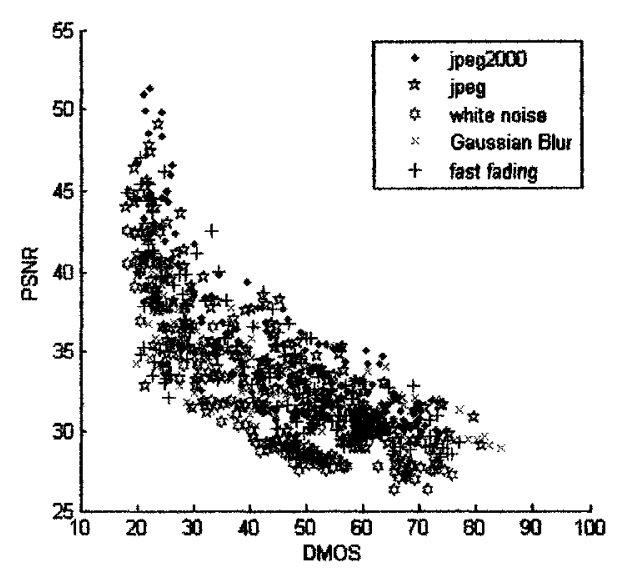

(a) PSNR

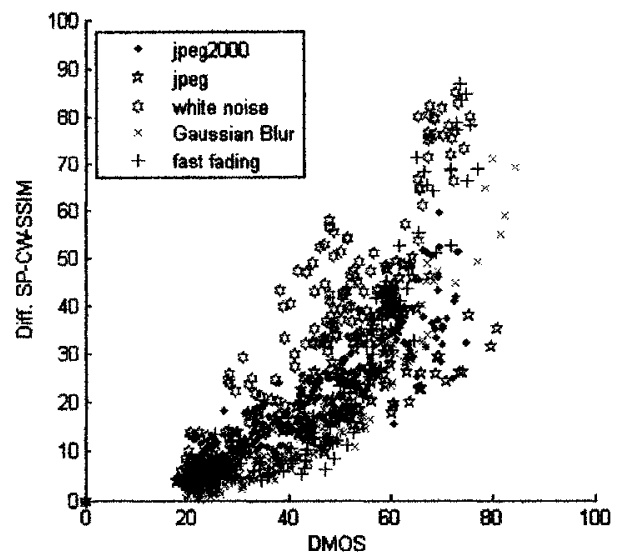

(c) SP-CW-SSIM

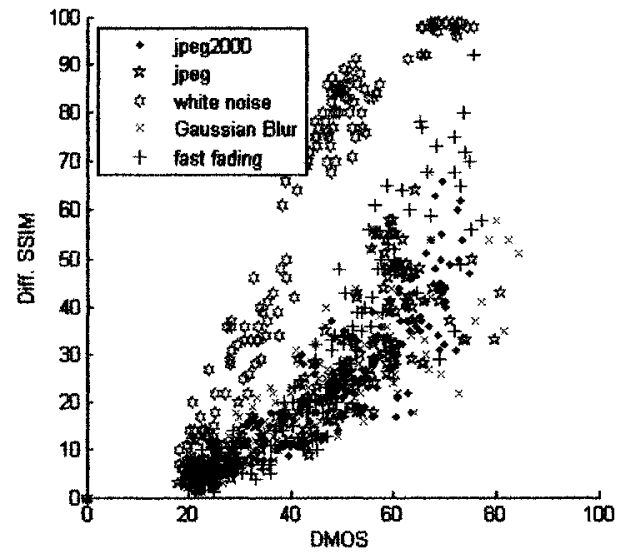

(b) SSIM

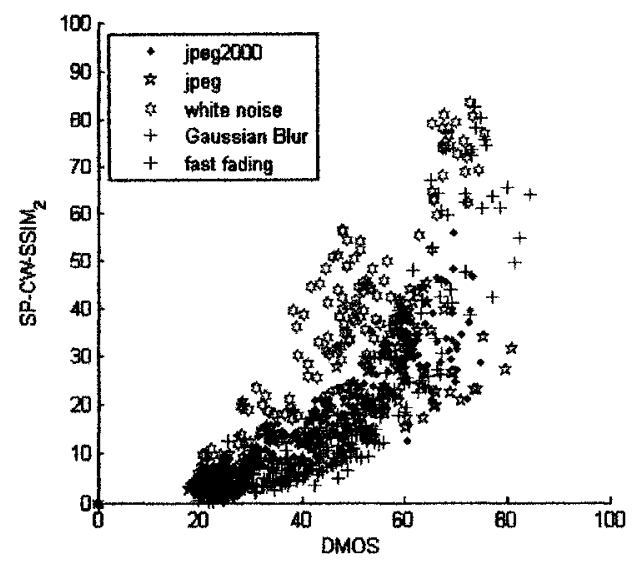

(d) SP-CW-SSIM 2

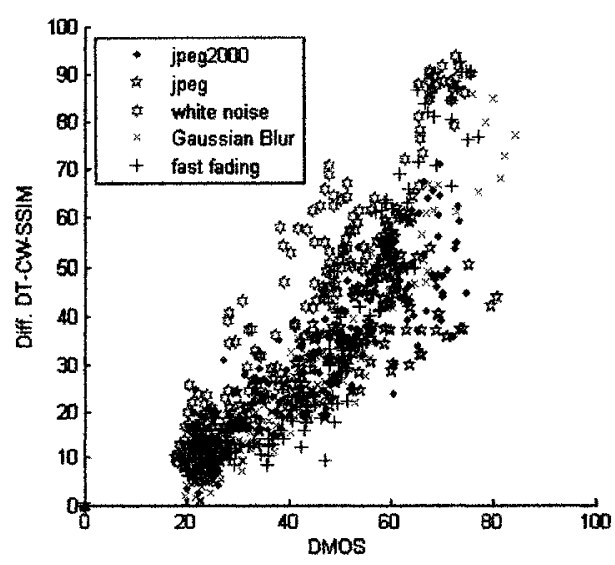

(e) DT-CW-SSIM

Figure 5.3: Scatter plots of the different difference quality results vs. the DMOS values for all the images in the LIVE image database. 


\subsection{Perceptually Weighted $\mathbb{C W}$-SSIM Index}

Since the HVS is less sensitive to artifacts in the higher frequency channels, Brooks et al. proposed the use of a perceptually weighted $\mathbb{C W}$-SSIM algorithm (W-CWSSIM) $[6,84]$. The W-CW-SSIM uses a perceptually weighted sum as opposed to using a simple average in calculating the overall image quality. Although the approach was discussed and results were presented in [6], the approach was not tested against a scored image database to truly compare its performance against the original $\mathbb{C W}$ SSIM algorithm.

To test the use of perceptually driven weights, we implemented the two algorithms W-SP-CW-SSIM and W-DT-CW-SSIM by adding perceptual weights to the SP-CWSSIM and DT-CW-SSIM algorithms, respectively. The algorithms follow the same steps as described before. However, instead of calculating the final image's quality as the mean of the different decomposition levels' quality values, it was calculated as a weighted sum. We loosely based the weights used in our implementations on those used in [6]. The used weights for both W-SP-CW-SSIM and W-DT-CW-SSIM algorithms were, from low to high spatial frequency, [0.1245, 0.2435, 0.3135, 0.3185] for the 4 level decomposition.

Both W-CW-SSIM algorithms were evaluated using the two test image collections Coll-I and Coll-II, with their results shown in Table 5.3 and Table 5.4, respectively. The scatter plots are presented in Figure 5.4 and Figure 5.5. 
Table 5.3: Correlation coefficient $(C C)$, non-linear mapped correlation coefficient $\left(\mathrm{CC}_{2}\right)$ and rank order correlation coefficient $(R O C C)$ of the weighted $\mathbb{C W}$-SSIM algorithms vs. DMOS for JPEG and JPEG2000 compressed images.

\begin{tabular}{|c||ccc|}
\hline Objective test & $C C$ & $C C_{2}$ & $R O C C$ \\
\hline \hline SP-CW-SSIM & 0.8262 & 0.9140 & 0.9334 \\
W-SP-CW-SSIM & 0.8887 & 0.9514 & 0.9521 \\
DT-CW-SSIM & 0.8866 & 0.9309 & 0.9378 \\
W-DT-CW-SSIM & 0.9141 & 0.9538 & 0.9496 \\
\hline
\end{tabular}

Table 5.4: Correlation coefficient $(C C)$, non-linear mapped correlation coefficient $\left(C C_{2}\right)$ and rank order correlation coefficient $(R O C C)$ of the weighted $\mathbb{C W}$-SSIM algorithms vs. DMOS for all images in release 2 of the LIVE image database.

\begin{tabular}{|c||ccc|}
\hline Objective test & $C C$ & $C C_{2}$ & ROCC \\
\hline \hline SP-CW-SSIM & 0.8262 & 0.9140 & 0.9334 \\
W-SP-CW-SSIM & 0.7987 & 0.9001 & 0.9270 \\
D'T-CW-SSIM & 0.8866 & 0.9309 & 0.9378 \\
W-DT-CW-SSIM & 0.8599 & 0.9236 & 0.9386 \\
\hline
\end{tabular}




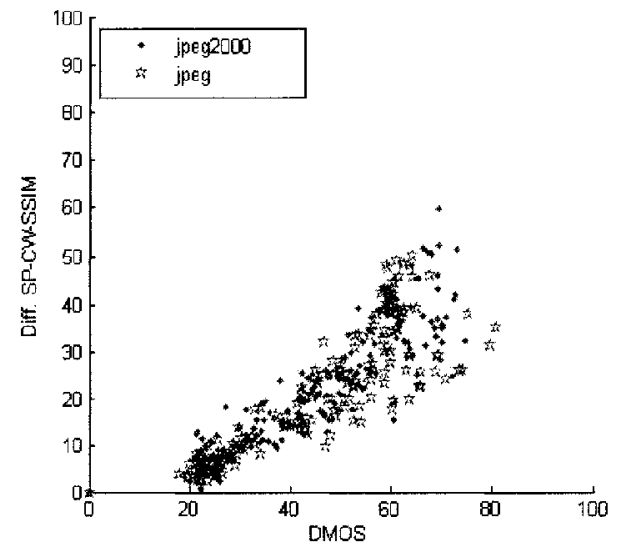

(a) SP-CW-SSIM

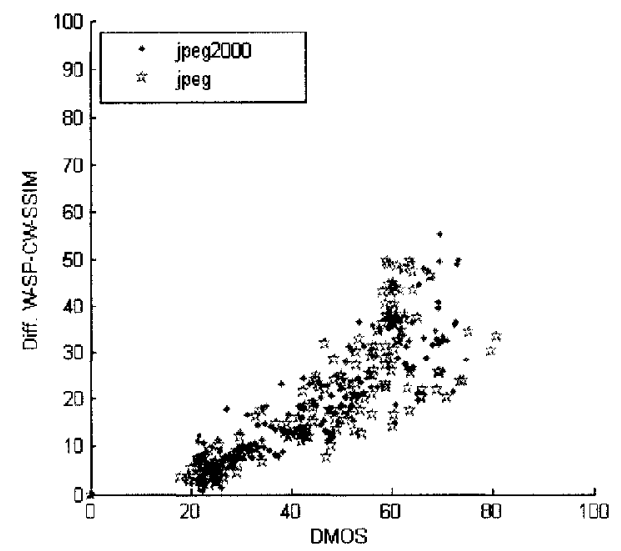

(c) W-SP-CW-SSIM

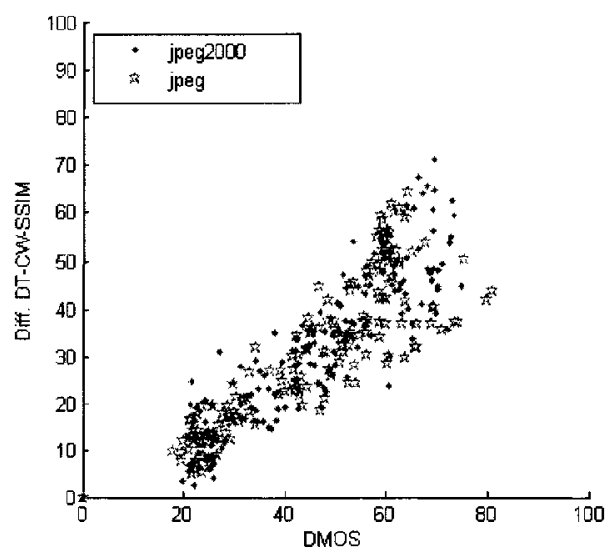

(b) DT-CW-SSIM

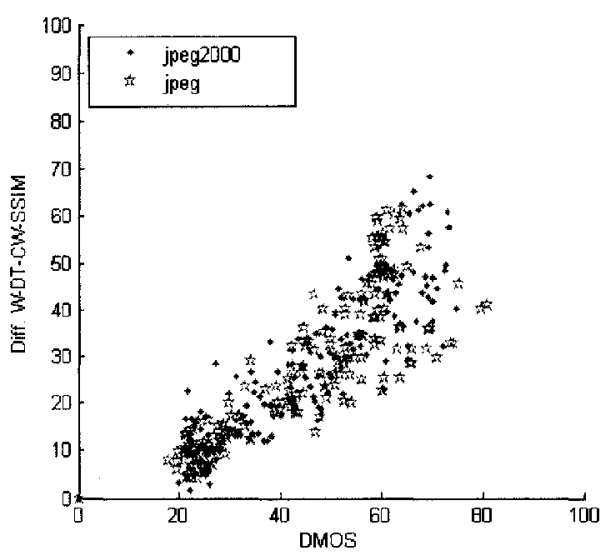

(d) W-DT-CW-SSIM

Figure 5.4: Weighted $\mathbb{C W}$-SSIM scatter plots of the SP-CW-SSIM and DT-CWSSIM algorithm's difference quality results vs. the DMOS values for all the JPEG and JPEG2000 images in the LIVE image database. 


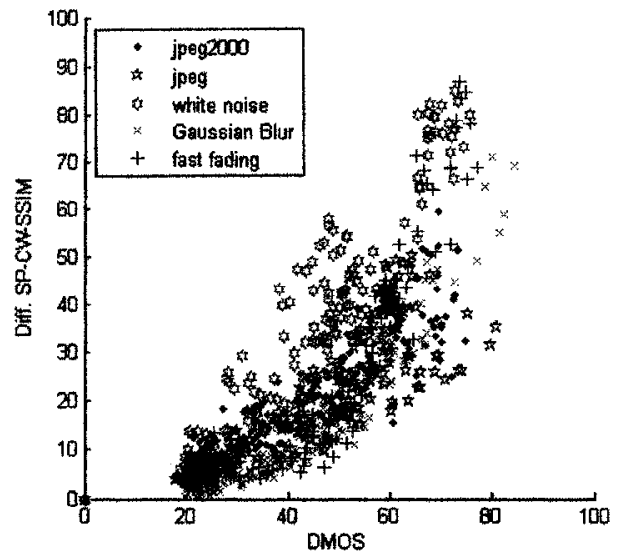

(a) SP-CW-SSIM

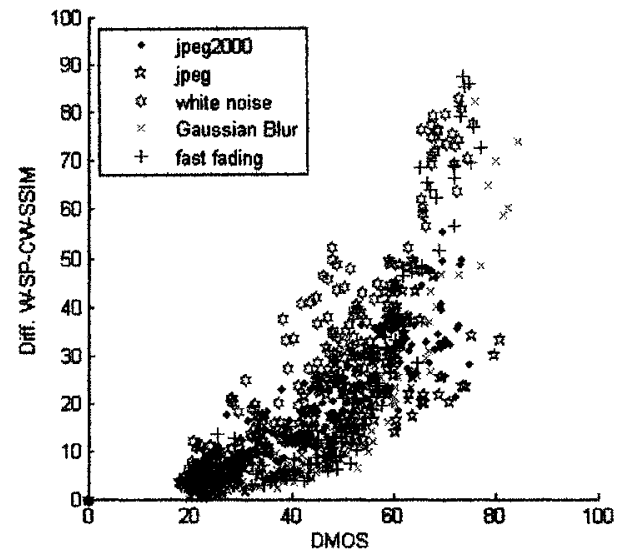

(c) W-SP-CW-SSIM

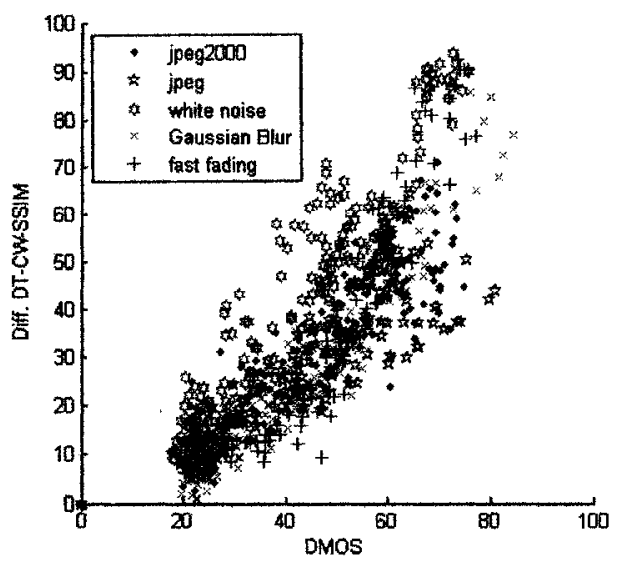

(b) DT-CW-SSIM

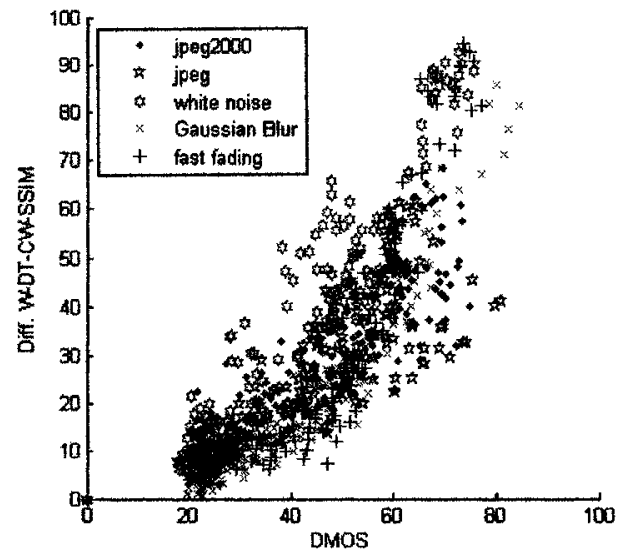

(d) W-DT-CW-SSIM

Figure 5.5: Weighted $\mathbb{C W}-$ SSIM scatter plots of the SP-CW-SSIM and DT-CWSSIM algorithm's difference quality results vs. the DMOS values for all images in the LIVE image database. 


\subsection{Discussion}

Our testing of the different algorithms shows that the SSIM algorithm performs better than the PSNR algorithm in both the compression data set test images, Coll-I, and the general distortion test images, Coll-II. Also, the different correlation results and scatter plots indicate that the CW-SSIM algorithm outperforms the SSIM algorithm as a general quality assessment algorithm.

Comparing the spatial SSIM algorithm with the different CW-SSIM implementations shows that the SSIM algorithms and the different CW-SSIM implementations have similar performance when tested against the JPEG and JPEG2000 images only. The CW-SSIM algorithm was developed to maintain the performance of the SSIM algorithm while increasing its tolerance to geometric distortion. As the used test image set did not contain any geometric distortions, the similarity in performance between the SSIM and the CW-SSIM implementations was expected. However, it can be clearly seen from the results in Table 5.2 that the CW-SSIM outperforms the SSIM index as a general quality prediction algorithm. Also, from the scatter plots in Figure 5.3, we see that the CW-SSIM algorithm behaves in a consistent manner with the different distortion types, unlike the SSIM algorithm. This observation indicates that due to the multi-scale decomposition property of the wavelet transform, the SSIM algorithm has better prediction accuracy in the complex wavelet domain that it does in the spatial domain.

Reducing the number of SP orientations used in the SP-CW-SSIM, from 16 to 6 , didn't negatively affect the overall quality assessment performance of the algorithm. on the other hand, reducing the number of orientation subbands per decomposition level significantly reduced the computation load of the algorithm. The scatter plots of the SP-CW-SSIM and SP-CW-SSIM 2 in Figure ?? and Figure ?? do not show any 
advantage in using a larger number of orientation subbands.

Both SP-CW-SSIM and DT-CW-SSIM implementations used four levels of decompositions with six orientation subbands each. Although both transforms have similar orientation subbands, with only $15^{\circ}$ rotation difference (see Figure 5.6), the DT-CWSSIM algorithm outperformed all the other implementations tested with both test image sets in Coll-I and Coll-II. The higher performance achieved from using the DT-CWT indicates that the DT-CWT can represent the decomposed signal better that the SP transform can, resulting in better overall structural similarity comparison and a better quality prediction.

it is important to note here that although we have only presented the algorithm's aggregated performance results, we have conducted algorithm evaluations using each individual distortion type in the LIVE image database separately. Evaluating the different algorithms using a single distortion type at a time showed that the DT$\mathbb{C W}$-SSIM implementation has a higher quality prediction accuracy, reflected by the results correlation with the images' subjective scores, against any distortion type.

Using perceptual weights with the $\mathbb{C W}$-SSIM algorithms increased all correlation values of both the SP-CW-SSIM and DT-CW-SSIM algorithms, as seen in Table 5.3, when tested only against the JPEG and JPEG2000 images. However, when the perceptually weighted $\mathbb{C W}$-SSIM algorithms were tested against the complete image set, the algorithms didn't show any performance increase over the regular CW-SSIM implementations (see 5.4). It is important to note here that the set of weights used in our experiments were loosely based on those used in [6]. We believe that the performance of the algorithms might still be increased if a study was performed to derive perceptual weights specific to the used transform and the viewing conditions for the images. 


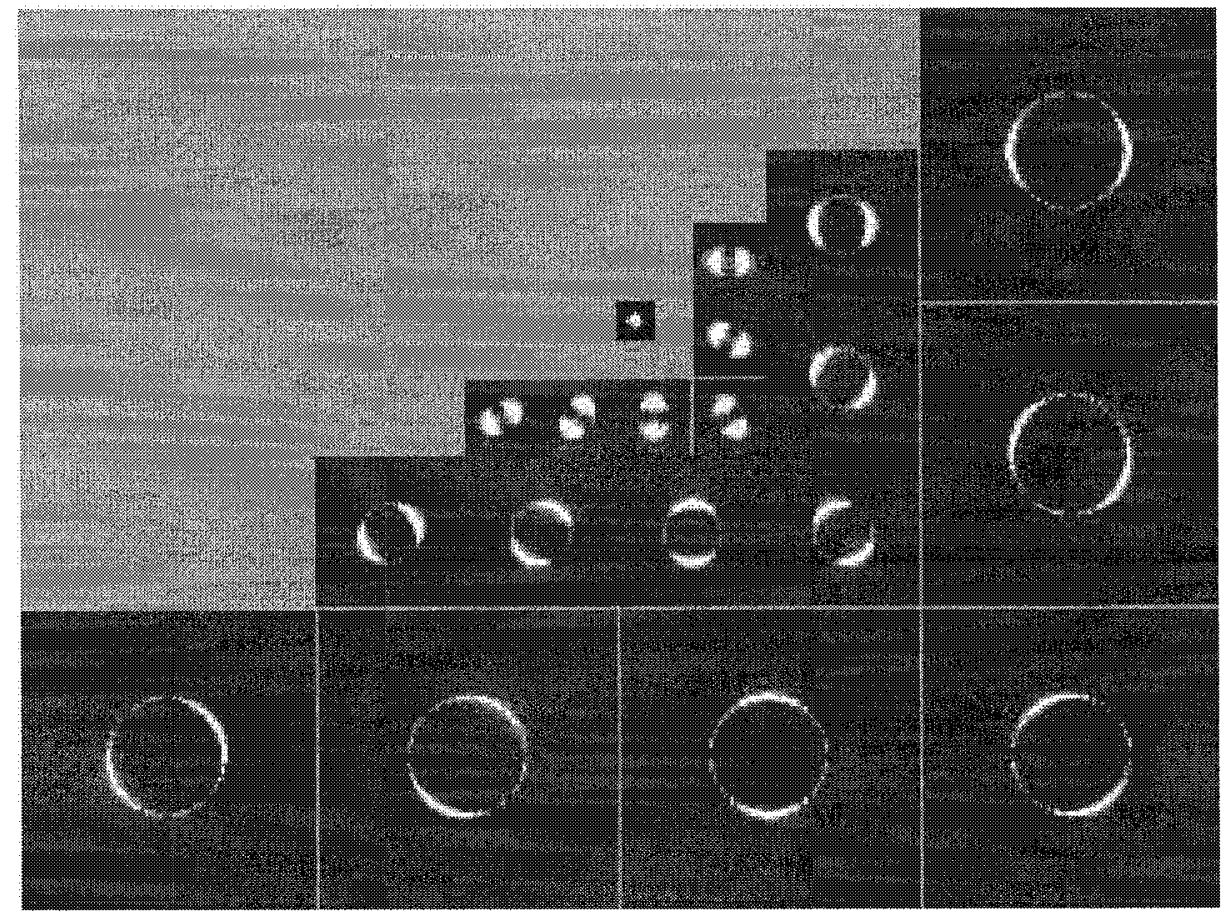

(a) Steerable pyramid decomposition

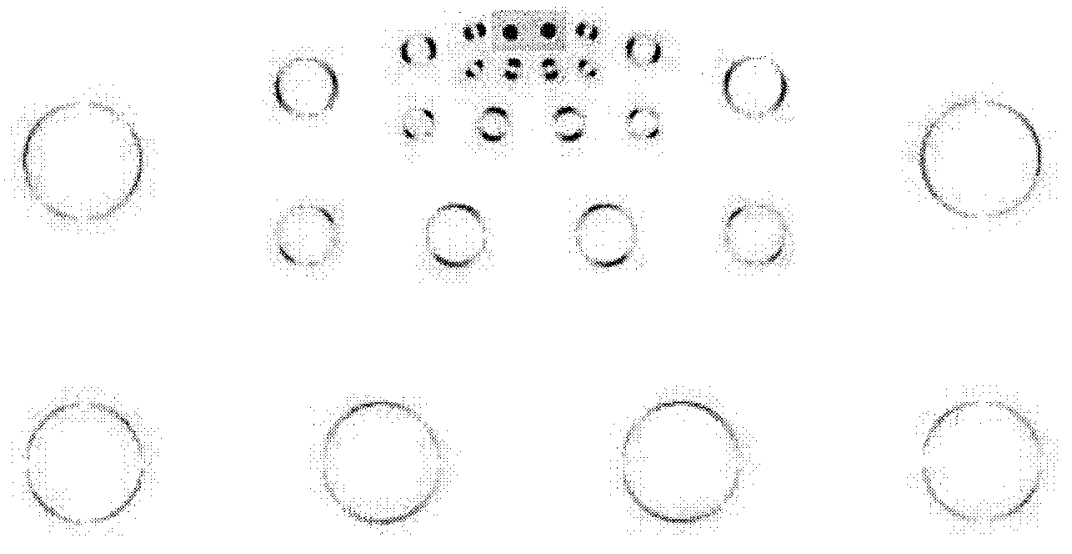

(b) DT-CWT decomposition

Figure 5.6: Disk image discomposed using (a) the steerable pyramid transform and (b) the DT-CWT. Both transforms used four levels of decomposition with six orientations at each level. Note that the original disk image is $256 \times 256$ pixels. The decomposition bands were normalized and scaled down for display purposes. The MATLAB implementations for both the SP and DT-CWT can be downloaded from [80] and [75], respectively. 
Looking at all the results from the different tests, we can clearly see that implementing the $\mathbb{C W}$-SSIM algorithm using the DT- $\mathbb{C W T}$ does not negatively affect the algorithm's prediction monotonicity. However, using the DT-CWT instead of the SP transform increases the algorithm's overall prediction accuracy. The higher performance obtained by using the DT-CWT along with the transform's extendability to higher dimensions makes it a perfect candidate for a video structural similarity assessment algorithm. 


\section{Chapter 6}

\section{Video Quality Assessment using the Dual-Tree Complex Wavelet SSIM}

With the high performance that the CW-SSIM algorithm showed in image quality assessment, and with the increased prediction accuracy gained from using the DT$\mathbb{C W T}$, using the $\mathbb{C W}$-SSIM algorithm with DT-CWT for video assessment becomes a possible option. In this chapter, we propose using the $3-\mathrm{D}$ DT-CWT with the $\mathbb{C W}$ SSIM algorithm for video quality assessment. We start by highlighting the benefit of using the 3D DT-CWT and discussing the proposed approach. After that, we talk about the video sequences used in evaluating the algorithm followed by implementation and testing results. The chapter will end with a discussion of the results.

\subsection{Why use the 3-D DT-CWT?}

The DT-CWT, although a newer transform, has shown potential in different areas of video processing. For instance, the $3-\mathrm{D}$ DT-CWT is reported to have good performance for video denoising [77] and video coding [76,82] algorithms.

The 3-D DT-CWT is a true 3-D non-separable transform (see Sec. 4.2). However, due to its design, the transform maintains the simplicity of the separable wavelet 
transform. With its true $3-\mathrm{D}$ nature, the $3-\mathrm{D}$ DT-CWT is capable of decomposing 3-D video signals into a number of subbands, each with its own distinct resolution and spatio-temporal orientation in space. The orientation bands of the 3-D DT-CWT are considered to be both orientation and motion selective for video. This allows the transform to reduce the overhead associated with video compression algorithms, by eliminating the need for the motion estimation/compensation steps.

Video quality assessment can be achieved by evaluating the sequence one frame at a time then taking the final video's quality as the average of all the frames' quality scores. Although such implementations might perform well for certain spatial distortions, such as pure random noise or general blur, they will not be able to capture and evaluate temporal artifacts in the video sequence. To address this problem, some video algorithms operate on a frame by frame basis but incorporate a motion estimation step to account for the temporal side of the video [19]. On the other hand, true video assessment algorithms operate on the video sequence as a 3-D block. Such algorithms tend to decompose the sequence based on HVS criteria using a separable 3-D transform. Due to simulating the HVS, these assessment algorithms tend to be very complex (see Chapter 2). Also, as they use separable 3-D decompositions, they will have limited feature extraction abilities.

Using the 3-D DT-CWT in the context of video quality assessment allows the quality algorithm to extract features from the video signal into unique subbands, allowing for better feature comparison. Also, as the transform's decomposition subbands are motion selective for video, the algorithm will be able to detect temporal artifacts in the video sequence without the need for explicit motion estimation. Another important advantage of the 3-D DT-CWT is its near shift invariant property. Such property can significantly reduce the assessment algorithm's complexity by reducing, if not eliminating, the need for a pre-processing registration step. 
Combining the features of the 3-D DT-CWT with those of the CW-SSIM algorithm allows us to build a simple, yet effective, video assessment algorithm. Such an algorithm not only operates as a stand-alone quality predictor, but can potentially be incorporated in the increasing number of dual-tree based video processing algorithms, for added quality control and optimization.

\subsection{Proposed Approach}

To combine the features of the DT-CWT with those of the CW-SSIM algorithm, we propose implementing the CW-SSIM algorithm using a 3-D DT-CWT at its core for video quality assessment. The video dual-tree complex wavelet structural similarity (V-DT-CW-SSIM) index, will be able to predict the quality of videos in a FR manner based on the sequences' $3-\mathrm{D}$ structure differences.

The V-DT-CW-SSIM as presented in Algorithm 2, starts by transforming the video sequence into the $\mathrm{YCbCr}$ color space. Each color component of both the reference and test video sequences is decomposed using the 3-D DT-CWT to four decomposition levels. The decomposition will result in a total of 1123 -D detail subbands, 28 from each decomposition level, each with its own unique resolution and orientation, and 4 low frequency subbands. As most of the structure information of the video sequences is extracted into the different detail subbands, we only use these subbands in the algorithm and discard the 4 low frequency subbands. After the decomposition step, the CW-SSIM algorithm is calculated between small 3-D sub-blocks extracted from corresponding locations from the reference and test video's detail subbands in a sliding window fashion. Similar to the implementations in Sec. 5.3, the color subblock's quality index, $Q$, is taken as the weighed sum of the three corresponding quality index values of the $\mathrm{Y}, \mathrm{Cb}$, and Cr components' sub-blocks, and is calculated 
as $Q=0.8 * Q_{Y}+0.1 * Q_{C b}+0.1 * Q_{C r}$. The different color sub-block quality indices are averaged to get the subband's quality index. The CW-SSIM indices of the 28 subbands of each decomposition level are averaged to obtain the level's quality index. The overall video quality index is calculated as the mean of the different level's quality values.

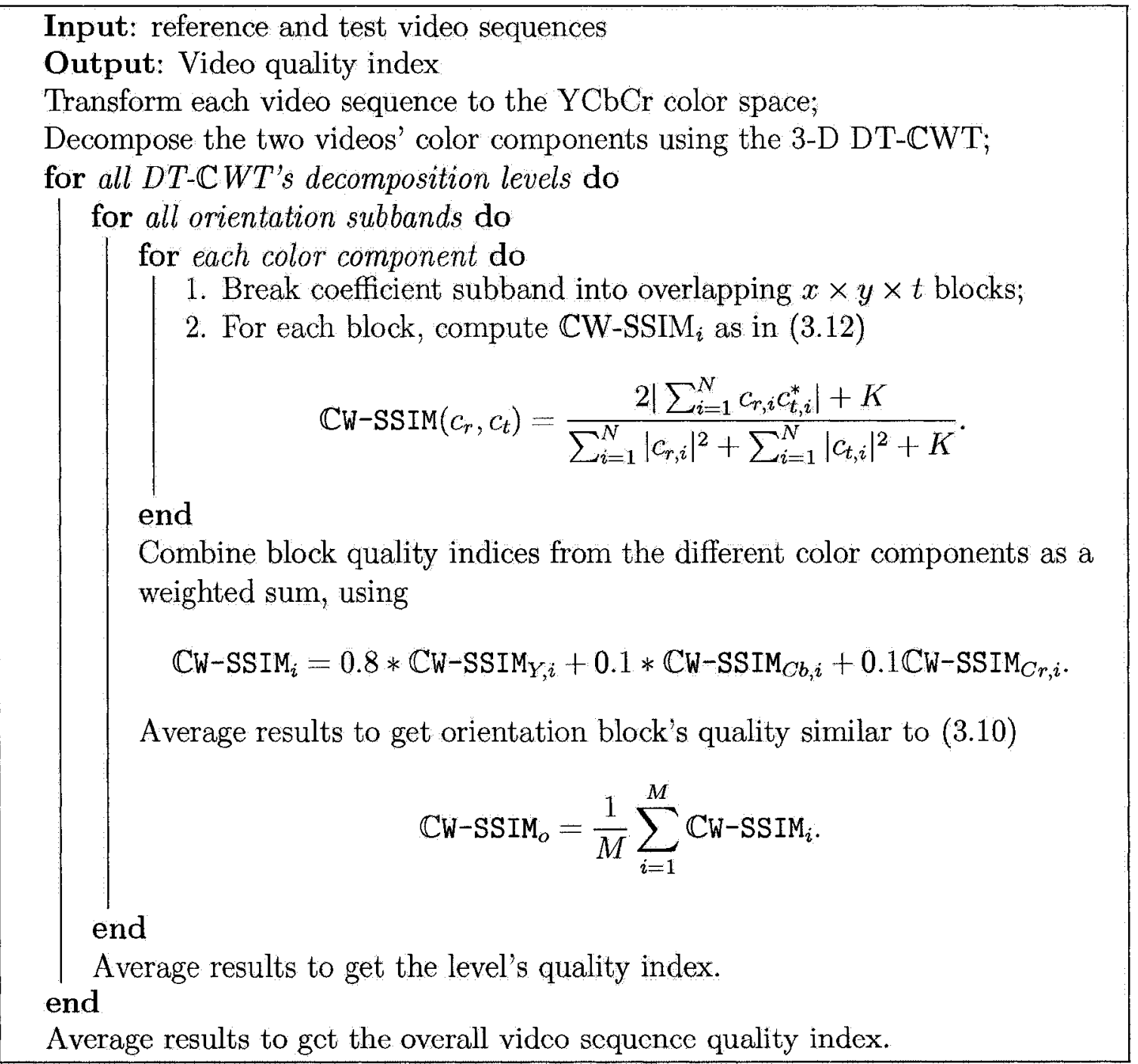

Algorithm 2: Video DT-CW-SSIM

The V-DT-CW-SSIM algorithm differs from the video-SSIM algorithm, discussed in Sec. 3.3, in that it operates on the video as a 3-D block and does not operate on 
a frame by frame basis. This allows the algorithm to detect any distortions in the temporal side of the video sequence. Since the 3-D DT-CWT subbands are motion selective, the algorithm is able to account for temporal artifacts without the need for explicit motion estimation or optical flow calculation as required by the V-SSIM algorithm (Sec. 2.7.8).

\subsection{Test Material}

To test the proposed approach, we obtained a set of 112 subjectively scored common intermediate format (CIF) test videos. The video sequences were processed by Nortel, in a collaborative effort with the video lab at Communications Research Center Canada (CRC), as part of the VQEG Multimedia (MM) project to evaluate objective quality assessment algorithms for CIF and QCIF video sequences ${ }^{1}$.

The set's test video cases were designed to reflect impairments from encoding compression and network transmission impairments (packet loss). The test video sequences used were generated from 7 reference CIF sequences, with 16 impaired sequences from each of the reference videos. The impairments in the test videos were generated through a combination of H.264 compression at bit rates of 720,256 , or $128 \mathrm{bps}$ frame rates of 30,20 , or $15 \mathrm{fps}$; and packet loss percentages of $0.0 \%, 0.5 \%$, $0.1 \%, 0.2 \%, 0.4 \%$, or $0.8 \%$. Not all the possible combinations were used in generating the test sequences, as some combinations were deemed unsensible for the real world. Examples on such combinations are combining low bit rate with high frame rate or high bit rate with low frame rate.

The test video sequences were scored using an absolute category rating (ACR) method. ACR is a single-stimulus method where the processed and unprocessed

\footnotetext{
${ }^{1}$ More on VQEG's multimedia project can be found in the project's Phase I report, [85].
} 
(reference) video sequences are presented alone. The scoring used a hidden reference (HR) approach. With the HR test, the reference video sequences are mixed with and presented among the processed video sequences, not as references but as stand alone sequences to be scored. The sequences were displayed in a random order with a few seconds in between for sequence rating. The scoring sheet used is similar to that shown in Figure 2.3(a). The DMOS values of the sequences were generated by subtracting each sequence's MOS result from that of its corresponding reference sequence. The full description of the subjective scoring method and environment can be found at [85].

\subsection{Implementation and Testing}

To test the proposed approach, we used the 3-D DT-CWT implementation provided by [75]. To reduce the computing and memory requirements, the implementation was designed to read the reference and test video sequences one group of pictures (GoP) at a time. Once a GoP is read, 3-D video blocks are randomly extracted from corresponding locations of the test and reference video sequences. For our test implementation, we set the block's spatial dimension to be $256 \times 256$ with its GoP depth set to 8 frames. The selected 3-D blocks are transformed from their color space to the $\mathrm{YCbCr}$ color space, resulting in one luminance and two chrominance 3-D blocks. The blocks are then decomposed using the 3-D DT-CWT at four levels of decomposition. The $\mathbb{C W}$-SSIM algorithm is applied to sub-blocks extracted from each decomposition subband, as discussed in Sec. 6.2. We used a dynamic sub-block size with a maximum size of $7 \times 7 \times 7$. We chose a dynamic window size to accommodate the size of the lower resolution decomposition subbands, which are smaller. The color sub-block's quality inde is taken as the weighed sum of the three corresponding 
color component quality index values as discussed in Sec. 6.2. The different color quality indices are averaged to obtain the decomposition subband's quality index. The quality index of each decomposition level is taken as the average of the quality indices of the 28 different subbands. The overall video quality index is taken as the mean of the different decomposition level's quality values.

Alongside the proposed 3D-DT-CW-SSIM algorithm, we implemented the videoSSIM algorithm, discussed in Sec. 3.3, and included it in our performance evaluation tests. All the quality prediction results were compared to their corresponding subjective DMOS values. As in the image DT-CW-SSIM evaluation tests, we used the $C C, C C_{2}$, and $R O C C$ to evaluate the algorithm's prediction accuracy and monotonicity. $C C_{2}$ is taken as Pearson's linear correlation coefficient between $\mathrm{DMOS}_{P}$ and DMOS values. DMOS $_{P}$ values are obtained through a nonlinear regression function as recommended by VQEG, [41], and descried in Sec. 5.3

Table 6.1 shows the results obtained by testing the video-SSIM and 3D-DTCW-SSIM algorithms against the $128 \mathrm{CIF}$ sequences described in Sec. 6.3. Due to the selected 3-D DT-CWT implementation's limitation in representing fast motion $[77,86]$, we ran a second test limiting the algorithms evaluation to low motion video sequences of the test video set. With that, the test sequences will consist of 2 video sequences form VQEG's MM project's sequences, CRC_SRC_bench_cif and CRC_SRC_birches1_cif (see Figure 6.1). None of the impaired sequences generated from the selected reference videos were eliminated from our testbed. The results from this test run are presented in Table 6.2. 


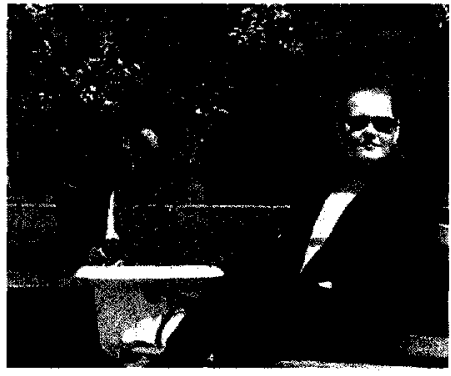

(a)

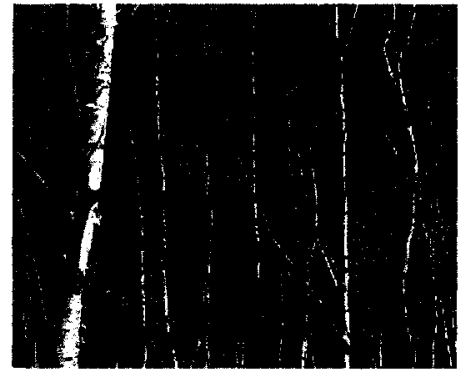

(b)

Figure 6.1: Thumbnails of two low motion video sequences from VQEG's MM project that were used in the algorithm's performance evaluation: (a) CRC_SRC_bench_cif and (b) CRC_SRC_birches1_cif.

Table 6.1: Correlation coefficient $(C C)$, non-linear mapped correlation coefficient $\left(C_{2}\right)$ and rank order correlation coefficient $(R O C C)$ of the 3D DT-CW-SSIM objective quality results vs. DMOS for VQEG's MM test video sequences.

\begin{tabular}{|c||ccc|}
\hline Objective test & $C C$ & $C C_{2}$ & ROCC \\
\hline \hline video-SSIM & 0.3776 & 0.4031 & 0.5111 \\
3D-DT-CW-SSIM & 0.6975 & 0.7096 & 0.7221 \\
\hline
\end{tabular}

Table 6.2: Correlation coefficient $(C C)$, non-linear mapped correlation coefficient $\left(C C_{2}\right)$ and rank order correlation coefficient $(R O C C)$ of the 3D-DT-CW-SSIM objective quality results vs. DMOS for the 2 low motion VQEG's MM test video sequences, the bench sequence and birches sequence.

\begin{tabular}{|c||ccc|}
\hline Objective test & $C C$ & $C C_{2}$ & $R O C C$ \\
\hline \hline video-SSIM & 0.7981 & 0.8007 & 0.7598 \\
3D-DT-CW-SSIM & 0.8095 & 0.8416 & 0.8510 \\
\hline
\end{tabular}




\subsection{Discussion}

Our algorithm evaluation clearly shows that the 3D-DT-CW-SSIM algorithm significantly outperforms the spatial video-SSIM algorithm. After adjusting the testbed video selection, to overcome the algorithm's limitation in representing fast motion, the performance of both tested algorithms increased. However, as shown in Table 6.2, the proposed approach still outperformed the video-SSIM algorithm. With that in mind, if the DT-CWT basis functions were designed to allow for better high motion representation, the algorithm's overall performance can be significantly enhanced.

From the collected test results, it can be concluded that the use of the 3-D DT$\mathbb{C W T}$ with the $\mathbb{C W}$-SSIM allows the algorithm to predict the quality of digital video sequences, while maintaining the simplicity of the $\mathbb{C W}$-SSIM algorithm. The 3D-CWSSIM algorithm, although limited at its current state to low motion sequences, shows great potential as a stand alone quality assessment algorithm or as quality control sub-algorithm, by embedding it into dual-tree video compression algorithms.

One of the research goals was to implement and evaluate a simple video quality assessment algorithm that does not contain a preprocessing registration step, nor does it contain an explicit motion estimation step. For that reason, all of the results obtained in the performance evaluation test process didn't include such steps. As the DT-CWT is a near shift invariant transform and not a full shift invariant one, the algorithm is only insensitive to small geometric distortions (small spatial shifts or rotations and around 2 frame shifts temporally, depending on the sequence motion level). With that in mind, adding a rough registration step to the algorithm, such as is included in a number of video quality assessment proponents being explored by VQEG, should increase the algorithm's quality prediction accuracy. 


\section{Chapter 7}

\section{Conclusions and Future Work}

\subsection{Conclusions}

With the increased number of digital image and video processing algorithms, there is high demand on quality assessment algorithms. To step away from costly subjective quality tests, objective quality assessment algorithms are developed to provide a tool that can be used for quality prediction, quality control, and algorithm optimization.

In general, objective quality assessment algorithms are designed to predict the quality of digital images and digital videos, based on properties of the HVS. As simulating the HVS results in complex algorithms, Wang et al.stepped away from the typical understanding of the HVS and developed the SSIM algorithm for objective image quality assessment. The SSIM algorithm, although a very simple assessment tool, has shown good performance in predicting the quality of digital images.

With the good performance of the SSIM algorithm, two algorithms were developed by others using SSIM for video quality prediction. The first, the video-SSIM algorithm [19], applied the SSIM algorithm to the digital video on a frame-by-frame basis and calculated the overall video's quality index as a weighted sum of the individual frame's

qualities. The frame quality weights used in the video-SSIM algorithm were based on 
the amount of motion of each frame. The second algorithm developed, the V-SSIM [2], was based on the complex wavelet extension of the SSIM algorithm, the CW-SSIM. This more complex algorithm used Gabor filters to decompose videos into HVS like channels and relied heavily on optical flow information in its quality assessment.

Simulating the HVS results in a complex assessment algorithm. However, the motion estimation, used in both available SSIM based video algorithms, adds complexity and overhead. There is a need for a simpler yet effective video assessment algorithm that avoids these complexities.

In this thesis, we proposed using the DT-CWT with the CW-SSIM algorithm for image quality prediction. Also, as the DT-CWT can be extended to provide a non separable 3-D transform with the simplicity of the separable transform, and with the 3-D D'T-CWT's directional and motion selectivity property, we proposed using the 3-D DT-CWT with the CW-SSIM algorithm, 3D-CW-SSIM for video quality assessment. As the 3-D DT-CWT is a motion selective transform, the 3D-CW-SSIM is capable of accounting for temporal artifacts in the video without the need for an explicit motion estimation step.

We evaluated the proposed 3D-DT-CW-SSIM algorithm using the LIVE image database. The performance of the proposed implementation was compared to that of the PSNR, the SSIM index, and the original CW-SSIM implementation using the steerable pyramid transform. Our test results showed that the CW-SSIM algorithm outperforms the original SSIM algorithm. Also, the performance evaluation shows that the dual-tree implementation of the $\mathbb{C W}$-SSIM algorithm has the highest performance with higher prediction accuracy than the steerable pyramid implementation for cross correlation and rank order cross correlation.

The 3D-DT-CW-SSIM algorithm was tested using video sequences from VQEG's MM project. The algorithm was implemented without any sequence registration or 
motion estimation steps. The algorithm's performance was compared to that of the video-SSIM algorithm and has shown a significantly higher performance over the video sequences. Although the performance of the 3D-DT-CW-SSIM algorithm was higher than that of the video-SSIM algorithm, the overall performance was not as high as expected. The reason for the lower than expected performance of the algorithm is due to the 3-D DT-CWT's limited ability in representing fast motion. To that, we have also evaluated the algorithm using videos from the video set that only contained low motion. Limiting the testbed to low motion videos increased the performance of the proposed algorithm, with the algorithm's correlation coefficient being 0.8416 as opposed to 0.8007 of the video-SSIM algorithm.

\subsection{Future Work}

With the complexity added by the HVS simulation, the image or video registration, and motion estimation, our goal was to develop and evaluate an objective video quality assessment tool that steps away from such complex features. To achieve our goal, we proposed and implemented the 3D-DT-CW-SSIM algorithm, resulting in a simple video assessment tool.

To further develop and enhance the performance of the proposed algorithm, research can be conducted to develop dual-tree wavelet basis that can better represent fast motion than the current 3-D implementation. Such transform can enhance the overall performance of the algorithm, allowing it to more accurately predict the quality of video sequences with a wider range of motion.

Another possible area of development is a study aimed at deriving perceptual weights for the 2-D and 3-D DT-CWT. As seen in Sec. 5.4, using a perceptually weighted sum to calculate the final image quality has the potential to increase the 
algorithms performance. To that, perceptual weights designed for the DT-CWT can enhance the performance of both the image and video implementations of the DTCW-SSIM algorithm.

A third avenue of development is adding a registration step to the algorithm. Adding a registration step to the image assessment algorithm will increase the complexity of the algorithm. This complexity is further elevated for video. However, as the DT-CWT is a near shift invariant transform, a rough registration step can be added to the algorithm as a pre-processing step. Such a step increases the algorithms tolerance to geometric distortions, that should not affect the final quality, increasing the overall performance of the algorithm.

One of the goals of the proposed implementation is to have a video assessment algorithm that accounts for temporal artifacts without explicitly estimating motion. However, if the algorithm is to be embedded with a compression algorithm that uses motion estimation, it can be developed to use the available motion information to compensate for and remove fast motion form the sequence, allowing for a good quality prediction. A motion compensated V-DT-CW-SSIM algorithm can be developed also as a stand alone algorithm, using a DT-CWT based motion estimation step. 


\section{List of References}

[1] S. Winkler, "Perceptual video quality metrics - A review," in Digital Video Image Quality and Perceptual Coding (H. R. Wu and K. R. Rao, eds.), ch. 5, pp. 155-180, CRC Press, 2006.

[2] K. Seshadrinathan and A. C. Bovik, "A structural similarity metric for video based on motion models," in Proc. of IEEE Intern. Conf. on Acoustics, Speech and Signal Processing, vol. 1, pp. 869-872, April 2007.

[3] Z. Wang, A. Bovik, H. Sheikh, and E. Simoncelli, "Image quality assessment: From error visibility to structural similarity," IEEE Trans. on Image Processing, vol. 13, pp. 600-612, April 2004.

[4] B. Girod, "What's wrong with mean-squared error?," in Digital Images and Human Vision (A. B. Watson, ed.), ch. 15, pp. 207-220, Cambridge, MA, USA: MIT Press, 1993.

[5] A. C. Brooks and T, N. Pappas, "Using structural similarity quality metrics to evaluate image compression techniques," in IEEE Intern. Conf. on Acoustics, Speech and Signal Processing, vol. 1, pp. I-873-I-876, April 2007.

[6] A. C. Brooks, X. Zhao, and T. N. Pappas, "Structural similarity quality metrics in a coding context: Exploring the space of realistic distortions," IEEE Trans. on Image Processing, vol. 17, pp. 1261-1273, Aug. 2008.

[7] S. Voran, "The development of objective video quality measures that emulate human perception," in Proc. of GLOBECOM, pp. 1776-1781, 1991.

[8] A. A. Webster, C. T. Jones, M. H. Pinson, S. D. Voran, and S. Wolf, "An objective video quality assessment system based on human perception," in SPIE Human Vision, Visual Processing, and Digital Display IV, pp. 15-26, 1993. 
[9] C. van den Branden Lambrecht and O. Verscheure, "Perceptual quality measure using a spatio-temporal model of the human visual system," in Proc. SPIE, vol. 2668, (San Jose, CA), pp. 450-461, Jan. 1996.

[10] C. van den Branden Lambrecht, "A working spatio-temporal model of the human visual system for image restoration and quality assessment applications," in Proc. of IEEE Intern. Conf. on Acoustics, Speech, and Signal Processing, vol. 4, pp. 2291-2294, May 1996.

[11] Y.-K. Lai, J. Li, and C.-C. J. Kuo, "A wavelet approach to compressed image quality measurement," in Conf. Record of the Thirtieth Asilomar Conference on Signals, Systems and Computers, vol. 2, (Pacific Grove, CA, USA), pp. 938-942, Nov. 1996.

[12] A. P. Bradley, "A wavelet visible difference predictor," IEEE Trans. on Image Processing, vol. 8, pp. 717-730, May 1999.

[13] Z. Yu and H. Wu, "Human visual system based objective digital video quality metrics," in Proc. of Intern. Conf. on Signal Processing, vol. 2, pp. 1088-1095, 2000 .

[14] N. Damera-Venkata, T. D. Kite, W. S. Geisler, B. L. Evans, and A. C. Bovik, "Image quality assessment based on a degradation model," IEEE Transactions on Image Processing, vol. 9, pp. 636-650, Apr. 2000.

[15] M. H. Pinson and S. Wolf, "A new standardized method for objectively measuring video quality," IEEE Transactions on Broadcasting, vol. 50, pp. 312-322, Sept. 2004 .

[16] W. Xuehui, L. Junli, and C. Gang, "An image quality estimation model based on HVS," in Proc. of IEEE TENCON, pp. 1-4, Nov. 2006.

[17] Z. Wang and A. Bovik, "A universal image quality index," IEEE Signal Processing Letters, vol. 9, pp. 81-84, Mar. 2002.

[18] Z. Wang, E. Simoncelli, and A. Bovik, "Multi-scale structural similarity for image quality assessment," in Proc. of 37th Asilomar Conf. on Signals, Systems and Computers, pp. 1398-1402, Nov. 2003.

[19] Z. Wang, L. Lu, and A. Bovik, "Video quality assessment based on structural distortion measurement," Signal Processing: Image Communication, vol. 19, pp. 121-132, Feb. 2004. 
[20] Z. Wang and E. Simoncelli, "Translation insensitive image similarity in complex wavelet domain," in Proc. of IEEE Intern. Conf. on Acoustics, Speech and Signal Processing, pp. 573-576, March 2005.

[21] D. C.-C. Lin and P. M. Chau, "Objective human visual system based video quality assessment metric for low bit-rate video communication systems," Proc. of IEEE Workshop Multimedia Signal Processing, pp. 320-323, Oct. 2006.

[22] I. Selesnick, R. Baraniuk, and N. Kingsbury, "The dual-tree complex wavelet transform," IEEE Signal Processing Magazine, vol. 22, pp. 123-151, Nov. 2005.

[23] F. W. Campbell, J. J. Kulikowski, and J. Levinson, "The effect of orientation on the visual resolution of gratings," The Journal of Physiology, vol. 187, pp. 427436, Nov. 1966.

[24] C. Blakemore and F. W. Campbell, "On the existence of neurons in the human visual system selectively responsive to the orientation and size of retinal images," Journal of Physiology, vol. 203, pp. 237-260, 1969.

[25] A. B. Watson, "DCT quantization matrices visually optimized for individual images," in Proc. SPIE, vol. 1913, pp. 202-216, Feb. 1993.

[26] R. L. DeValois and K. K. DeValois, Spatial Vision. Oxford University Press Inc, June 1988.

[27] J. Daugman, "Spatial visual channels in the Fourier plane," Vision Research, vol. 24 , no. 9, pp. 891-910, 1984.

[28] A. B. Watson, "Temporal sensitivity," in Handbook of Perception and Human Performance (K. R. Boff, L. Kaufman, and J. P. Thomas, eds.), vol. 1, ch. 6, Wiley-Interscience, 1986.

[29] S. T. Hammett and A. T. Smith, "Two temporal channels or three? A reevaluation," Vision Research, vol. 32, pp. 285-291, Feb. 1992.

[30] A. B. Watson, "The cortex transform: Rapid computation of simulated neural images," Comput. Vision Graph. Image Process., vol. 39, no. 3, pp. 311-327, 1987.

[31] A. S. H. Senane and D. Barba, "The computation of visual bandwidths and their impact in image decomposition and coding," in Proc. of Intern. Conf. on Signal Processing Applications and Technology, pp. 766-770, 1993. 
[32] S. Winkler, "A perceptual distortion metric for digital color video," in Proc. SPIE, pp. 1-4, 1999.

[33] G. E. Legge and J. M. Foley, "Contrast masking in human vision," J. Optical Society of America, vol. 7, pp. 1458-1471, Dec. 1980.

[34] E. Peli, "Contrast in complex images," J. Optical Society of America, vol. 7, pp. 2032-2040, Oct. 1990.

[35] S. Winkler and P. Vandergheynst, "Computing isotropic local contrast from oriented pyramid decompositions," in Proc. of IEEE Intern. Conf. on Image Processing, vol. 4, pp. 420-424, 1999.

[36] S. Daly, "The visible differences predictor: An algorithm for the assessment of image fidelity," in Digital Images and Human Vision (A. B. Watson, ed.), ch. 14, pp. 179-206, Cambridge, MA, USA: MIT Press, 1993.

[37] S. Daly, Z. Wenjun, L. Jin, and L. Shawmin, "Visual masking in wavelet compression for JPEG2000," in Proc. SPIE Image and Video Communications and Processing, vol. 3974, (San Jose CA), pp. 66-80, Jan 2000.

[38] A. B. Watson, R. Borthwick, and M. Taylor, "Image quality and entropy masking," in Proc. SPIE, Human Vision and Electronic Imaging II, vol. 3016, pp. 2$12,1997$.

[39] A. Ninassia, O. Le Meura, P. Le Calletb, and D. Barbab, "On the performance of human visual system based image quality assessment metric using wavelet domain," in Proc. SPIE, Conference Human Vision and Electronic Imaging XIII (B. E. Rogowitz and T. N. Pappas, eds.), vol. 6806, pp. 10-21, 2008.

[40] Y. Yuan, D. Feng, and Y.-Z. Zhong, "The causation and solution of ringing effect in DCT-based video coding," Intern. Journal of Applied Mathematics and Computer Sciences (IJAMCS), vol. 2, no. 1, pp. 19-24, 2006.

[41] VQEG, "FRTV phase II report, final report from the video quality experts group on the validation of objective models of video quality assessment," tech. rep., VQEG, August 2003.

[42] Z. Wang, H. R. Sheikh, and A. C. Bovik, "Objective video quality assessment," in The Handbook of Video Databases: Design and Applications (B. Furht and O. Marqure, eds.), ch. 41, pp. 1041-1078, CRC Press, Sept. 2003. 
[43] J. L. Mannos and D. J. Sakrison, "The effects of a visual fidelity criterion on the encoding of images," IEEE Trans. Information Theory, vol. 20, pp. 525- 536, Jul. 1974.

[44] J. Lubin, "A visual discrimination model for imaging system design and development," in Vision Models for Target Detection and Recognition (E. Peli, ed.), vol. 2, ch. 10, pp. 245-283, World Scientific, 1995.

[45] C. C. Taylor, Z. Pizlo, J. P. Allebach, and C. A. Bouman, "Image quality assessment with a Gabor pyramid model of the human visual system," in Proc. of Intern. Symposium on Electronic Imaging Science and Technology, vol. 3016, (San Jose CA), pp. 58-69, Feb. 1997.

[46] A. B. Watson, J. Hu, and J. F. McGowan III, "DVQ: A digital video quality metric based on human vision," J. of Electronic Imaging, vol. 10, no. 1, pp. 20$29,2001$.

[47] A. B. Watson, G. Y. Yang, J. A. Solomon, and J. Villasenor, "Visibility of wavelet quantization noise," in IEEE Trans, on Image Processing, vol. 6, pp. 1164-1175, Aug. 1997.

[48] J. Oh, S. I. Woolley, T. N. Arvanitis, and J. N. Townend, "A multistage perceptual quality assessment for compressed digital angiogram images," IEEE Trans. on Medical Imaging, vol. 20, pp. 1352-1361, Dec. 2001.

[49] Q. Li and Z. Wang, "Video quality assessment by incorporating a motion perception model," in Proc. of IEEE Intern. Conf. on Image Processing, vol. 2, pp. II $-173-$ II -176 , Oct. 2007.

[50] H. Rushmeier, G. Ward, C. Piatko, P. Sanders, and B. Rust, "Comparing real and synthetic images: Some ideas about metrics," the 6th Eurographics Workshop on Rendering, In Proceedings of, 1995.

[51] M. J. Gervais, J. L. O. Harvey, and J. O. Roberts, "Identification confusions among letters of the alphabet," J. Experimental Psychology. Human Perception and Performance, vol. 10, pp. 655-666, Oct. 1984.

[52] W. T. Freeman and E. H. Adelson, "The design and use of steerable filters," IEEE Trans. Pattern Analysis and Machine Intelligence, vol. 13, pp. 891-906, Sep. 1991. 
[53] M. W. Cannon, "A model of mechanisms mediating spatial pattern perception," in Proc. of the Human Factors Society $36 t h$ Annual Meeting, (Santa Monica, CA, USA), p. 1430 1434, 1992.

[54] CCIR, "Subjective assessment of the quality of television pictures," Report 405-5 Vol. XI-I, Dubrovink, 1986.

[55] P. Lindh and C. van den Branden Lambrecht, "Efficient spatio-temporal decomposition for perceptual processing of video sequences," in Proc. of Intern. Conf. on Image Processing, vol. 3, (Lausanne, Switzerland), pp. 331-334, Sep. 1996.

[56] P. Teo and D. Heeger, "Perceptual image distortion," in Proc. of IEEE Intern. Conf. on Image Processing, Proceedings, vol. 2, (Austin, TX), pp. 982-986, Nov. 1994.

[57] A. B. Watson, "Toward a perceptual video quality metric," in IS6T/SPIE Conference on Human Vision and Electronic Imaging III, vol. 3299, (San Jose, California), pp. 139-147, NASA Ames Research Ctr, Jan 1998.

[58] M. A. Georgeson and J. M. Georgeson, "Facilitation and masking of briefly presented gratings: time-course and contrast dependence," Vision Research, vol. 27, no. 3, pp. 369-379, 1987.

[59] E. Hering, Zur Lehre vom Lichtsinn. Vienna, Austria: Carl Gerolds \& Sohn, 1878.

[60] L. M. Hurvich and D. Jameson, "Some quantitative aspects of an opponent-colors theory. ii. brightness, saturation, and hue in normal and dichromatic vision.," $J$. Optical Society of America, vol. 45, no. 8, pp. 602-616, 1955.

[61] L. M. Hurvich and D. Jameson, "An opponent-process theory of color vision," Psychological Review, vol. 64, pp. 384-404, 1957.

[62] E. P. Simoncelli, W. T. Freeman, E. H. Adelson, and D. J. Heeger, "Shiftable multi-scale transforms," IEEE Trans Information Theory, vol. 38, pp. 587-607, Mar 1992.

[63] VQEG, "Final report from the video quality experts group on the validation of objective models of video quality assessment," tech. rep., VQEG, March 2000. 
[64] S. Wolf and M. H. Pinson, "Video quality measurement techniques," Report 02-392, National Telecommunications and Information Administration (NTIA), June 2002.

[65] S. Yao, W. Lin, E. Ong, and Z. Lu, "A wavelet-based visible distortion measure for video quality evaluation," in Proc. of IEEE Intern. Conf. on Image Processing, pp. 2937-2940, Oct. 2006.

[66] D. J. Fleet and A. D. Jepson, "Computation of component image velocity from local phase information," Intern. J. of Computer Vision, vol. 5, pp. 77-104, Aug. 1990.

[67] A. B. Watson, "Visual detection of spatial contrast patterns: Evaluation of five simple models," Optics Express, vol. 6, pp. 12-33, Jan. 2000.

[68] Z. Wang, L. Lu, and A. Bovik, "Video quality assessment using structural distortion measurement," in Proc. of Intern. Conf. on Image Processing, vol. 3, pp. $65-68,2002$.

[69] H. R. Sheikh, M. F. Sabir, and A. C. Bovik, "A statistical evaluation of recent full reference image quality assessment algorithms," IEEE Transactions on Image Processing, vol. 15, pp. 3440-3451, Nov. 2006.

[70] H. R. Sheikh and A. C. Bovik, "Image information and visual quality," IEEE Transactions on Image Processing, vol. 15, pp. 430-444, Feb. 2006.

[71] N. Kingsbury, "The dual-tree complex wavelet transform: A new technique for shift invariance and directional filters," in Proc. 8th IEEE DSP Workshop, 1998.

[72] N. Kingsbury, "A dual-tree complex wavelet transform with improved orthogonality and symmetry roperties," in Proc. Intern. Conf. on Image Processing, vol. 2 , pp. $375-378,2000$.

[73] I. Selesnick, "Hilbert transform pairs of wavelet bases," IEEE Signal Processing Letters, vol. 8, pp. 170-173, June 2001.

[74] I. Selesnick, "The design of approximate hilbert transform pairs of wavelet bases," IEEE Transactions on Signal Processing, vol. 50, no. 5, pp. 1144-1152, 2002 .

[75] S. Cai and K. Li, "Matlab implementation of wavelet transforms." http://taco.poly.edu/WaveletSoftware/index.html. 
[76] B. Wang, Y. Wang, I. Selesnick, and A. Vetro, "Video coding using 3d dual-tree wavelet transform," EURASIP J. on Image and Video Processing, vol. 2007, p. 15 pages, Jan. 2007.

[77] I. W. Selesnick and K. Y. Li, "Video denoising using 2d and 3d dual-tree complex wavelet transforms," in Wavelet Appl Signal Image Proc. X (Proc. SPIE 5207, pp. $607-618,2003$.

[78] S. Gupta, M. P. Sampat, M. K. Markey, A. C. Bovik, and Z. Wang, "Facial range image matching using the complexwavelet structural similarity metric," IEEE Workshop on Applications of Computer Vision, pp. 4-9, Feb. 2007.

[79] E. P. Simoncelli and W. T. Freeman, "The steerable pyramid: a flexible architecture for multi-scale derivative computation," in Proc. of Intern. Conf. on Image Processing, vol. 3, pp. 444-447, Oct 1995.

[80] E. P. Simoncelli, "The steerable pyramid." http://www.cns.nyu.edu/ eero/steerpyr/.

[81] T. H. Reeves and N. G. Kingsbury, "Overcomplete image coding using iterative projection-based noise shaping," in Proc. of Intern. Conf. on Image Processing, vol. 3, pp. 597-600, June 2002 .

[82] J. Yang, B. Wang, Y. Wang, and W. Xu, "Video coding using 3-d anisotropic dual-tree wavelet transform," in Proc. of IEEE Intern. Conf. on Multimedia and Expo, pp. 1371-1374, July 2007.

[83] H. R. Sheikh, Z. Wang, L. Cormack, and A. C. Bovik, "Live image quality assessment database release 2." http://live.ece.utexas.edu/research/quality.

[84] A. C. Brooks and T. N. Pappas, "Structural similarity quality metrics in a coding context: Exploring the space of realistic distortions," Proc. of SPIE, Human Vision and Electronic Imaging XI, vol. 6057, pp. 299-310, Feb. 2006.

[85] VQEG, "Final report from the video quality experts group on the validation of objective models of multimedia quality assessment, phase I," tech. rep., VQEG, Sep. 2008.

[86] F. Shi and I. W. Selesnick, "Video denoising using oriented complex wavelet transforms," in Proc. of IEEE Intern. Conf. on Acoustics, Speech, and Signal Processing, vol. 2, pp. 949-952, May 2004. 